University of Louisville

ThinkIR: The University of Louisville's Institutional Repository

Electronic Theses and Dissertations

8-2006

\title{
An examination of social presence in an online learning environment.
}

Susan J. Crim

University of Louisville

Follow this and additional works at: https://ir.library.louisville.edu/etd

\section{Recommended Citation}

Crim, Susan J., "An examination of social presence in an online learning environment." (2006). Electronic Theses and Dissertations. Paper 291.

https://doi.org/10.18297/etd/291

This Doctoral Dissertation is brought to you for free and open access by ThinkIR: The University of Louisville's Institutional Repository. It has been accepted for inclusion in Electronic Theses and Dissertations by an authorized administrator of ThinkIR: The University of Louisville's Institutional Repository. This title appears here courtesy of the author, who has retained all other copyrights. For more information, please contact thinkir@louisville.edu. 


\title{
AN EXAMINATION OF SOCIAL PRESENCE IN AN ONLINE LEARNING ENVIRONMENT
}

\author{
By \\ Susan J. Crim \\ B.S., East Tennessee State University, 1974 \\ M.S., University of Tennessee, 1985
}

\begin{abstract}
A Dissertation
Submitted to the Faculty of the

Graduate School of the University of Louisville

in Partial Fulfillment of the Requirements

for the Degree of
\end{abstract}

\section{Doctor of Philosophy}
Department of Education, Leadership and Human Resource Development University of Louisville
Louisville, Kentucky

August, 2006 

CCopyright 2006 by Susan J. Crim

All rights reserved 


\section{AN EXAMINATION OF SOCIAL PRESENCE IN AN ONLINE LEARNING ENVIRONMENT}

By

Susan J. Crim

B.S., East Tennessee State University, 1974

M.S., University of Tennessee, 1985

\section{A Dissertation Approved on}

May 25, 2006

by the following Dissertation Committee:

Dissertation Director 


\section{ACKNOWLEDGEMENTS}

I could not have realized this accomplishment without the invaluable support and encouragement of a number of people-mentors, colleagues, and family. First, I would like to express my sincere appreciation to my dissertation advisor and mentor, Dr. Thomas G. Reio, Jr., for his guidance, patience, sense of humor, and steady assurance, throughout, not only this process, but my entire program of study. He is a strong role model of a true professional whose trenchant scholarship strives to contribute to the field of human resource education. His positive attitude and dedication to students makes the learning experience a truly memorable one. Dr. Reio guided me through this process with his unwavering interest in my study and his untiring encouragement. From him I learned to become a true scholar; both as a critical thinker and a researcher.

I would also like to thank the other committee members, Dr. Joe Petrosko, Dr. Mike Boyle, Dr. Carolyn Rude-Parkins, and Dr. Randall Wells for their comments and valuable assistance in the completion of my dissertation research. In addition, I would also like to express my appreciation and gratitude to the Delphi Center for Teaching and Learning and particularly, Joni Allison, whose assistance and expertise made the instrument development and data collection possible. I also owe thanks to the distance education online students who volunteered to participate in the surveys. It is their contributions that need to be recognized if this study provides any significance to the field. 
Most importantly, I would like to express my sincere gratitude, appreciation, and love to my husband and best friend, Gary Crim, for his constant understanding, patience, unconditional love, and valuable support throughout this process when there did not seem to be a light at the end of anything and the world would seem to close in. His strong belief in me and his constant encouragement have been invaluable. Also, my appreciation is extended to the members of my family, friends and colleagues who continually encouraged and supported me in achieving this goal. My gratitude can never be fully expressed of how much you helped to hearten me and promote my efforts. I feel truly blessed to have each of you in my life and I am pleased to share this accomplishment with you. 


\title{
ABSTRACT \\ AN EXAMINATION OF SOCIAL PRESENCE IN AN \\ ONLINE LEARNING ENVIRONMENT
}

\author{
Susan J. Crim
}

May 25, 2006

The distance education literature is lacking studies investigating the construct of social presence, the value placed on it by online learners, and whether its existence in text-based environments is necessary for learning, satisfaction, and contributing to course retention. The purpose of this study was to explore learner perceptions and experiences of the learning process within the Web-based online learning medium in terms of social presence. More specifically, it examines the relationship among learners' perceptions of social presence in asynchronous online courses and how it relates to satisfaction with learning, whether course activities perceived as having high levels of social presence also have high levels of satisfaction and quality of learning, and whether perceptions of social presence and satisfaction with learning affects their likelihood of enrolling in future online courses.

The research design of this study utilized an online survey administered to 280 students enrolled in online courses of nine disciplines; both undergraduate- and graduatelevel at a large urban university. Open-ended questions from the online survey were examined as well to help inform and support the findings from the quantitative data. Data were analyzed using correlations, ANOVAs, and hierarchical regression analysis. 
The findings demonstrated that students' perceived social presence was statistically, significantly, and positively related to their overall perceived satisfaction with learning in online courses. Furthermore, students' perceived social presence was statistically, significantly, and positively related to their perception of quality of and satisfaction with learning for each of the five course activities examined in this study. The hierarchical regression analysis suggested that perceived social presence contributed substantially more incremental variance to the decision to enroll again in an online course than the satisfaction with learning variable. Overall, the theoretical model including social presence and satisfaction with learning explained 18 percent of the variance in the dependent variable. The potential implications for theory and practice for online course designers and instructors are provided. 


\section{TABLE OF CONTENTS}

PAGE

ACKNOWLEDGEMENTS ..................................................................... iii

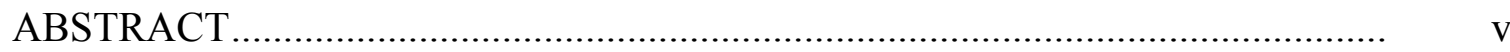

LIST OF TABLES ...................................................................................... viii

\section{CHAPTER}

I. INTRODUCTION.................................................................... 1

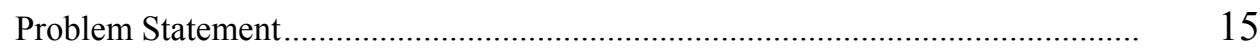

Purpose of Study .................................................................................... 16

Research Questions ................................................................................ 17

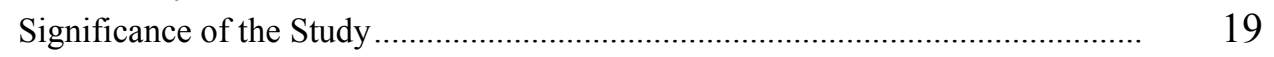

II. LITERATURE REVIEW ............................................................... 23

Overview of Online Learning ...................................................................... 23

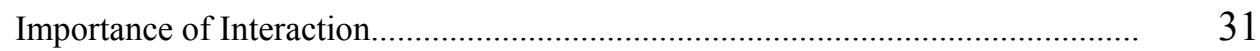

Social Presence ........................................................................................... 39

Communication Cues of Interaction, Immediacy and Intimacy........................ 46

Relationship between Social Presence and Communication Cues..................... 52

Community of Learners .............................................................................. 53

Socio-cultural and Constructivist Theory ........................................................... 57

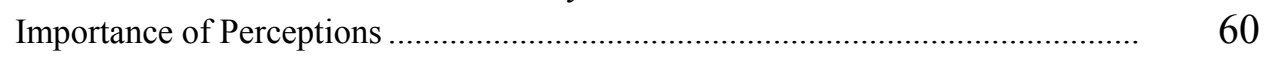

Implications of Social Presence for Online Learning ............................................ 63

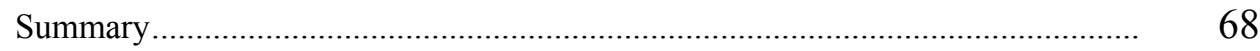

III. METHODOLOGY ................................................................. $\quad 70$

Research Design and Selection of Participants.................................................. 71

Internet Survey Research ......................................................................... 75

Advantages and Disadvantages.......................................................................... 76

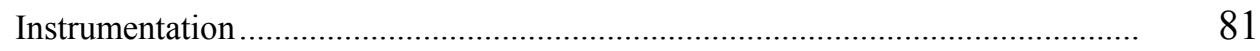

Data Collection ........................................................................................... 94

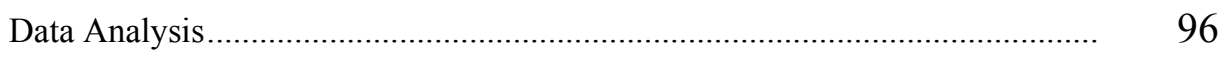

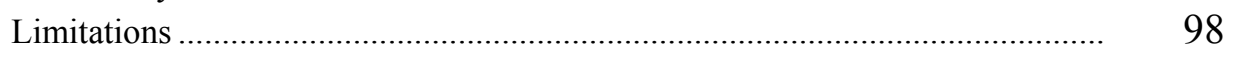

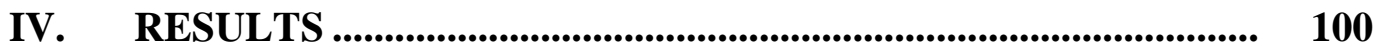

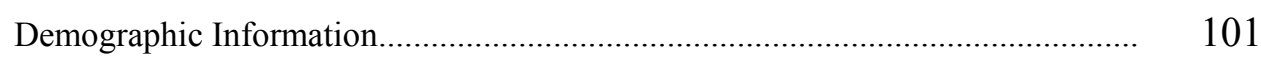

Survey Instrument ............................................................................... 106

Research Question 1 .............................................................................. 107

Research Question 2 …..................................................................... 112

Research Question 3 ................................................................................ 117 
Qualitative Data Analysis ......................................................................... 120

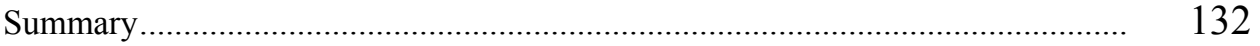

V. DISCUSSION ........................................................................... 134

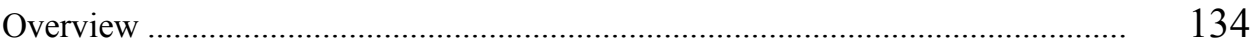

Relationship between Social Presence and Satisfaction with Learning ................. 136

Relationship between Social Presence and Quality of Learning in Individual

Course Activities ....................................................................................... 138

Relationship between Social Presence, Satisfaction with Learning, and Intent to Enroll or Persist in an Online Course

Relationship between Social Presence, Constructivist Theory, and Community

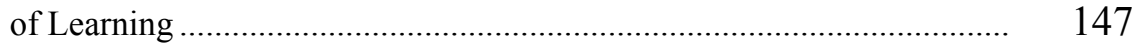

Implications of Social Presence in Online Learning ............................................. 148

Limitations and Recommendation for Future Research........................................ 154

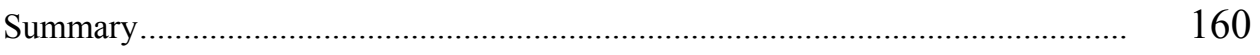

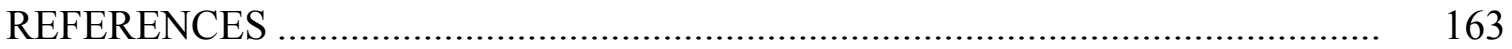

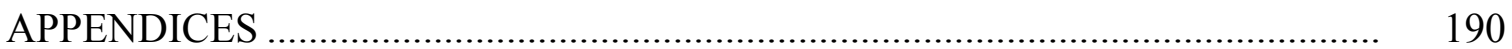

Appendix A: Pre-notification of Online Survey ……...................................................... 190

Appendix B: Notification of Online Survey................................................................. 192

Appendix C: Social Presence Online Survey ................................................................. 194

Appendix D: Motives for Taking Online Course .............................................................. 199

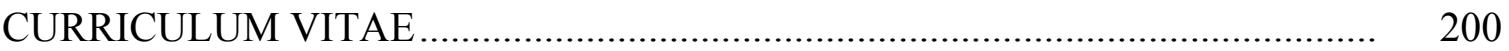




\section{LIST OF TABLES}

TABLE PAGE

$1 \quad$ Scoring Rule for Instrument....................................... 88

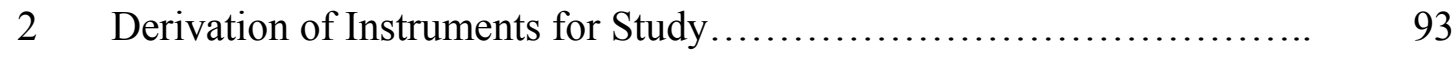

3 Timeline and Tasks for Data Collection................................ 95

$4 \quad$ Statistical Procedures and Analysis of Data............................. 97

$5 \quad$ Demographic Information.......................................... 105

6 Means, Standard Deviations, and Correlations of Research Variables.... 108

7 Means, Standard Deviations, and Correlations for Demographic Items With Students' Perceptions of Social Presence and Satisfaction with Learning....................................................... 111

$8 \quad$ Means and Correlations for Individual Course Activities................. 113

9 Correlations between Social Presence, Satisfaction with Learning, Quality of Learning, and Course Activities............................. 116

10 Hierarchical Regressions with Social Presence and Satisfaction with Learning Predicting Intent to Enroll in a Future Online Course...........

11 Frequencies and Percentages of Students' Responses Regarding Beneficial Activities of Online Courses....................................

12 Frequencies and Percentages of Students' Responses Regarding Reason For Choosing Particular Course Activities as Most Beneficial........... 122

13 Frequencies and Percentages of Students' Responses Regarding Amount In Interaction with Instructor. 


\section{CHAPTER I}

\section{INTRODUCTION}

The advancement of technology is changing instructional delivery in the world of higher education as the Internet has become one of the up-and-coming technologies for the delivery of distance education (Huang, 1997). Online learning has been promoted as being more cost effective and convenient than traditional education environments as well as providing opportunities for more learners to continue their education in various settings (Oliver, 1999). Because of these features, the use of synchronous-communication that occurs between two or more people in real-time-- and asynchronous online learning-- a time-delayed interaction that does not require participants be online simultaneously-- and the Internet has significantly changed the way learning is delivered and facilitated by allowing for the conversion of traditional courses into Web-based courses (Jiang \& Ting, 2000). Although the combination of the computer and various communication technologies facilitate the learning and interaction processes between the instructor and the learners at a distance, it is the time-delayed and place-independent asynchronous online learning mode that is of interest to this study.

As evident from the body of literature in distance learning, the integration of computers and the World Wide Web (WWW) has transformed the communication and learning process in higher education both nationally and internationally (Trentin \& Benigno, 1997). Distance education programs are making use of computer networking 
and conferencing media to facilitate the communication process. For example, the British Open University, considered a pioneer in distance education, first used computer conferencing to manage enrollment of over a thousand distance learners (Harasim, 1995). Likewise, universities in the United States have implemented computer conferencing to supplement instructor-learner interactions (Harasim, 1995). Although distance learning is considered an international phenomenon, the focus of this study examines the communication and learning process that takes place online in the United States.

As access to the Internet and World Wide Web has continued to grow, Web-based learning has continued to expand. With approximately half of the households in the United States (or 150 million people) connected to the Internet, an estimated two million students are taking postsecondary courses that are fully delivered online (Picciano, 2002). Today, about two-thirds of the 3,200 accredited four-year colleges and universities in the United States supplement their campus courses with classes via the Internet (Clarke, 1999). Many observers believe that the growth in distance education in the years ahead will continue to occur primarily through the use of the Internet and Web-based courses (Mehrotra, Hollister, \& McGahey, 2001). With the emergence of the Internet, it has become possible to promote interaction within a technological medium and provide a cost-effective learning environment (Woods \& Baker, 2004). However, the effectiveness of online courses particularly in relation to individual learner needs, perception, retention, and learning outcomes is frequently questioned (Phipps \& Merisotis, 1999).

The communication, cognitive/social psychology, and distance education literature identifies interaction among students as critical in learning and cognitive development (Gorham, 1988; Kelly \& Gorham, 1988; Sharan, 1980; Slavin, 1983; 
Vygotsky, 1978). There is a belief that high levels of interaction, particularly those which promote social engagement, can have positive effects on the learning experience.

It is known from research on learning processes in a traditional face-to-face learning environment that development of social climate is important in order to make students feel like they are a part of the learning community, thus contributing to students' motivation, involvement, learning outcomes, and contentment (Wegerif, 1998). The literature strongly indicates both students' and instructors' desire for contact (Rezabeck, Meyers, \& Edwin, 1992). Although it is recognized that there is a need to focus on instructor attitudes and specific factors affecting their participation in online learning, this study specifically focuses on the importance of the perceived learning and student satisfaction with the online learning experience.

Social interaction is a natural human need and is acknowledged as an important factor in the development of learning processes (Vygotsky, 1986). Vygotsky's emphasis on the social dimension in construction of knowledge has led many instructors to pay as much attention to how students learn as to what they are learning. A common element for learning in a traditional classroom is the social interaction between learner-instructor and learner-learner (Picciano, 2002). It is claimed to be important in technology-mediated learning situations (Harasim, 1995) because of an absence of non-verbal cues and texton-screen that provides a limited means of rich dialogue. Active approaches to effective learning emphasize learning as a social process that takes place through communication and interaction with others (Hiltz, Coppola, Rotter, Turoff, \& Benbunan-Fich, 2000). Indeed, several studies suggest a positive correlation between socially supportive online environments and cognitive learning (Gunawardena, 1995; Kanuka \& Anderson, 1998; Rovai, 2002; Swan, Shea, Frederickson, Pickett, Pelz, \& Maher, 2000; Wegerif, 
1998). For example, Swan et al. (2000) examined factors that contributed to perceived learning and student satisfaction in an online asynchronous graduate cohort course through a satisfaction survey and determined that students who reported the highest levels of interaction with the instructor and other learners also reported the highest levels of social engagement, participation and perceived learning in their Web-based course. In addition, Kanuka and Anderson (1998) found through observations and surveys that social-cognitive processes among participants in an online forum included significant time engaged in social interchange.

Nevertheless, most studies that examined Web-based learning reported no difference in learning achievement between students taking Web-based courses and students enrolled in traditional learning environments; moreover, some students indicated dissatisfaction with the online environment (Carswell, 2000; Collins, 2000; Kearsley, 2000). Although the same results could be said to be true for face-to-face classrooms, the educational technology literature abounds with arguments for and against the learning achievement and satisfaction gained among students in distance education compared to students in conventional settings (Kulik \& Kulik, 1991).

As the research findings of learning and student satisfaction in an online environment are mixed at best, it is important to assess the learner's perceptions of a Web-based learning environment as to the value they place on the importance of interaction and socialization among participants in the learning process. Many researchers have stressed the need for, and value of, Web-based learning environments that provide active and engaging activities for learners as they argue that students should have opportunities to construct knowledge rather than just being exposed to the transmission of knowledge (Hong, Lai, \& Holton, 2003). Such an emphasis on and 
perceived benefits associated with interpersonal social dynamics is consistent with the constructivist framework that argues that promoting student interactions is integral to effective online learning. For example, Harasim (1989) in her examination of online courses drew a similar conclusion about the value of student interaction and knowledge construction by stating, "knowledge building occurs as students explore issues, examine one another's arguments, agree, disagree, and question positions" (p.53). As a result, new ways of understanding the material emerge as a result of student contact with new or different perspectives based upon collaboration among their peers and the building of a community of learners. In other words, learning is not only active but also interactive.

Many educators in higher education are more cognizant of the need to shift their thinking about a traditional teaching-centered model to a more active learner-centered approach in both the face-to-face learning environment and the distance education learning environment (Harasim, 1990; Kaye, 1992; Malikowski, 1997). The rationale behind this shift in theoretical perspectives of learning is based on socio-constructivist theory that focuses on the issues of how best to design and conduct courses that fosters social interactions among learners and encourages construction of knowledge with others in a learning community (Barr \& Tagg, 1995; Slavin, 1990, Vygotsky, 1986). The teacher no longer assumes the authoritative position and, instead, becomes a facilitator and mentor in the learning process (Vygotsky, 1986). Learning becomes a collaborative act among the participants, rather than the simple reception of information.

A few studies have emphasized the importance of examining social factors that impact communication, interaction and learning in telecommunications and computermediated based systems (Hackman \& Walker, 1990; Lea, 1992; McIsaac \& Gunawardena, 1996; Sanders \& Wiseman, 1990; Walther, 1992) as distance learning 
students face a very different learning environment than those in a traditional classroom. The concept of the classroom where students meet to interact with other learners and the instructor no longer exists in the virtual technology-mediated model. Students in Webbased learning environments do not have an instructor physically present, but instructorlearner interaction perhaps should still take place. Whereas students in traditional settings can more readily interact with their instructors and peers, these same types of interactions must be carefully planned and structured by instructors of distance education (Parker, 1999). According to Northrup (2001), interaction must be intentionally designed into a Web-based course, as the interaction does not simply happen because the materials and tasks are presented to students for their consumption.

Even though distance education may be considered to be an independent learning environment, it is not the same as an isolated learning environment. Failure to consider the relational dynamics in the online setting may produce greater feelings of isolation, reduced levels of satisfaction, less participation, poor academic performance, and increased attrition among distance learners (Woods \& Baker, 2004). Collaboration with faculty and other students can be a strong motivating force for learning (Johnson \& Johnson, 1999), and it is important to provide a strong social dynamic in conjunction with the delivery of content (Palloff \& Pratt, 1999).

Some researchers claim that online learning may not be as effective as traditional classroom learning because of its lack of face-to-face and non-verbal cues. (Bullen, 1998; Ward, 1998). In general, students' interactions are restricted to text only messages on screen. This may reduce the depth and extent of the communication and interaction that occurs thereby decreasing the breadth and scope of knowledge gained. For example, students may not formulate questions that extend beyond the course content or initiate 
discussion for further clarification as usually occurs in the traditional face-to-face environment. The online environment is sometimes considered as a medium for social isolation.

In a traditional classroom, sensory cues such as voice inflection, facial expressions, and other body language indicate presence and facilitate communication. The lack of nonverbal cues might impact interpersonal relations (Short, Williams, \& Christie, 1976) and can contribute to a sense of disconnectedness. For example, Bullen (1998) conducted a case study examining participation and critical thinking in a college level undergraduate course utilizing computer-mediated conferencing. The case study showed that some students felt disconnected from others in this type of learning environment, citing lack of facial expressions and other features common to a traditional classroom environment. Without the interaction of face-to-face teaching, it appears to be easy for students working in an online learning environment to accept material passively and become observers of the course rather than engaging with the instructor and other students in the learning process. Such learning is particularly counter-productive when it comes to developing cognitive skills such as problem-solving, analyzing and critical thinking. Because of the lack of traditional communication cues and sense of isolation in the online learning environment, researchers have been interested in examining ways to improve this environment through enhancing the social context and interaction of online learners and instructors (Tu \& McIsaac, 2002).

Interaction alone, however, is insufficient to create a positive social dynamic in the online learning environment. It is possible for a student to post a message online while not necessarily feeling that she or he is part of a group. The ability to work effectively in groups is at the heart of social presence theory and of interest to those 
involved in creating a more social online learning environment and communities of learners (Stein \& Wanstreet, 2003). Research has shown that social presence--the degree of awareness of another person that occurs in a mediated environment-- is the most important perception that occurs in social context and is an important key to understanding communication and interaction in the field of distance learning (Gunawardena \& Zittle, 1997; Rourke, Anderson, Garrison, \& Archer, 2001). Gunawardena (1995) argues that social presence is necessary to enhance and improve effective instruction in both traditional and technology-based learning environments. A lack of social presence may lead to higher levels of frustration, a more critical attitude toward the instructor's effectiveness, and a lower level of perceived effective learning (Rifkind, 1992).

In the examination of interaction, the theory and concept of social presence or a sense of being in a place and belonging to a group is receiving increased attention (Picciano, 2002). As online learning is conducted with individuals independent of place and time, this altered learning environment does not preclude the need to establish learning relationships with online learners and instructors. Social, interactive, and affective dimensions of the learning experience remain powerful determinants of successful learning because they can enhance communication, improve teaching, increase student interest in content matter, and serve as a way to construct knowledge and negotiate meaning (Rodriguez, 1995; Wulf, Hanor, \& Bulik, 2000).

Social presence in an online course has been the subject of a number of articles redefining and categorizing this concept (Gunawardena, 1995; Rourke, Anderson, Garrison, \& Archer, 2001; Short et al., 1976; Tu, 2002; Wulf et al., 2000). For example, many researchers (Kanuka \& Anderson, 1998; McIsaac, Blocher, Mahes, Vrasidas, 1999) 
have investigated student and or instructor perceptions of online courses, only focusing on the interaction dimension. It has recently been found that to increase the level of online interaction, the degree of social presence must be increased (Tu, 2000).

$\mathrm{Tu}$ (2000), for example, conducted a study on the dimensions of social presence in the online learning environment through surveys and observations to understand social presence in an online learning environment from a student's point of view. Based on the author's findings, a high level of social presence was necessary to enhance, foster and increase interaction. Interaction can be fostered by communication styles that may impact social presence (relaxed, friendly, attentive, encouraging), by the learners' perceptions of the online environment, and by the activities or tasks (written assignments, group projects, online presentations) in which the learners engage.

Interaction is a key factor in distance education and is an important component of a successful instructional program. Whether students are interacting face-to-face or at a distance, their success may be a result of well-designed instructional strategies that take into consideration the factors that will promote interaction and enhance users' perceptions of learning and their satisfaction of their learning environment. Both Hillman (1999) and Moore (1989) recommend designing activities that allow learners an opportunity to interact productively that could contribute to frequency of interaction and formation of a learning community.

For example, Wagner (1997) suggests considering the course goals and objectives in order to effectively design an interactive learning community. Wagner proposed several strategies for design consideration that include such course activities as group work, discussion forums, and problem-solving. Aside from applying these strategies, Wagner (1997) suggests maintaining the learners' involvement, encouraging student 
collaboration, providing timely feedback, and implementing various instructional strategies in consideration of different learner styles. If the degree of social presence affects the level of interaction and participation (Tu, 2000), then it is important to examine the strategies that promote interaction among learners and those that enhance perceptions of a user's social presence.

Past studies on human interpersonal communication identified "intimacy" and "immediacy" as attributes that enhance social presence (Argyle \& Dean, 1965; Wiener \& Mehrabian, 1968). More recent research in the field of distance education and communication (Christophel, 1990; Gunawardena \& Zittle, 1997; McIsaac \& Gunawardena, 1996) has begun now to focus on the use of asynchronous communication, contending that these two attributes along with "interactivity" play an important role in forming interpersonal relationships in the communication process. This relationship addresses successful learning experiences in terms of intimacy-- sense of close connection one feels in a relationship (Argyle \& Dean, 1965), immediacy-- psychological distance between a communicator and the recipient of the communication (Wiener \& Mehrabian, 1968), and interactivity-- the activities in which users engage and the communication styles they use in computer-mediated communication (Gunawardena, 1995; Norton, 1986; Tu, 2000). Together these form the construct of social presence, defined as "the salience of the other in a mediated communication and the consequent salience of their interpersonal interaction" (Short et al., 1976, p. 65). This is interpreted as the degree to which a person is perceived as "real" in mediated communication (Gunawardena, 1995; Lombard \& Ditton, 1997).

Examination of the Short et al. (1976) definition indicates that although social presence may be a property of the medium, this characteristic is derived from the affect 
of the medium on the perceptions of the participants, and on their interpersonal interactions. Therefore, it must also be related to a property of that perception or interaction. For example, Biocca et al. (2001) defines social presence as pertaining to the user, but also relates it to the interaction and the medium in that it is a temporary judgment of interaction that is limited or augmented by the medium.

The overall goal for creating social presence in any learning environment, whether it is online or face-to-face, is to create a level of comfort in which people feel at ease around the instructor and the other participants. Without this goal being achieved, "the learning environment can turn to one that is not fulfilling or successful for the instructors and the learners" (Aragon, 2003, p. 60). Research suggests that there is a lack of dialogue among distance learning students, which impact "the quality and integrity of the educational process" (Sherry, 1996, p. 5). When the environment is lacking social presence, the participants may see it as impersonal and, in turn, the amount of information shared with others decreases (Leh, 2001). As a result, the lack of social presence could lead to more frustration, dissatisfaction and less participation in learning.

Closely associated with learner satisfaction is retention with distance delivered courses. The geographic and physical separation of students in programs offered at a distance may also contribute to higher dropout rates than in traditional face-to-face programs. Carr (2000) noted that dropout rates are often 10 to 20 percentage points higher in distance education courses than in traditional courses. Studies have indicated that in some cases more than $50 \%$ of online learners drop out before they complete their course (Hart, 2003). Some studies show that retention in online courses has been a puzzling problem for educational institutions. There is a greater likelihood that a student will not complete courses and stay enrolled in an online course than in a traditional 
course (Palloff \& Pratt, 2001). Other studies show staggering statistics that student dropout rates in online course are as high as $35-50 \%$ as compared to traditional classes (Lynch, 2001). There is also evidence that suggests online learning may be less effective in the industrial sectors as reports on the incomplete training via e-learning can reach as high as $80 \%$ (Bonk, 2004; Moshinskie, 2001).

Physical separation has a tendency to reduce the sense of community, giving rise to feelings of disconnection (Kerka, 1996), isolation, distraction, and lack of personal attention (Besser \& Donahue, 1996; Hardy \& Boaz, 1997; Twigg, 1997). Tinto (1993) emphasized the importance of community in reducing the dropout rate when he theorized that students will increase their levels of satisfaction and the likelihood of persisting in a college course or program if they feel involved and develop relationships with other members of the learning community.

Raising social presence in online environments may help to create perceptions of quality related to the experience on the part of the learner (Newberry, 2001). High levels of social presence create a learning environment that can support cognitive (critical thinking, problem-solving, scaffolding, reflection) and affective (collaboration, feedback/reinforcement, exchanging resources and information) learning objectives by making group interactions that are perceived as warm, collegial, engaging, and intrinsically rewarding (Rourke et al., 2001).

The literature on quality issues in distance learning (Phipps, et al., 1998; Swan et al., 2000) suggests that data on measures of interaction and presence should be used in studying student performance. Performance data can be in the form of tests, course grades, written assignments, projects and satisfaction surveys. Northrup (2001) suggests that learner perceptions of the efficacy of interaction and social presence can have 
significant effects on learning outcomes as it may affect learner performance, such as increased interest, participation, collaboration, and active learning.

Because social presence is a perception, it can and does vary from individual to individual. It can also be situational and vary across time for the same individual, making it a very complex construct for study. Researchers and educators need to examine its nature for the purpose of understanding interaction and social presence in an online environment. According to White (2000), Web-based instruction is as effective as face-to-face instruction in regards to academic achievement (as measured by final course grades), but not necessarily the same in regard to the quality of instruction due to an absence of student interaction, interest and participation.

Likewise, Bullen (1998) stresses the need for more studies that examine online learning from the learners' perspective. The experience of individual learners, as they negotiate this new way of learning, communicating, and sharing information, has not been a large part of educational technology research (Saye, 1997), but learner experiences may affect the efficacy and viability of online courses. Understanding learner experiences and perspectives is important because students most likely make individual decisions about the value and sustainability of online learning for themselves based upon their experiences and impressions of those experiences. For example, learner perceptions of social presence and its value in relation to mediated interaction and participation could provide insight as to whether asynchronous online courses have the capability to convey social presence and whether or not its existence is necessary for learner satisfaction, achievement, and the decision to enroll in future Web-based courses.

Studies conducted on social presence, for the most part, have been in traditional classrooms. Results from these studies indicated that social presence is a significant 
factor in instructional effectiveness and quality, positively affecting learning, learner satisfaction, achievement, and motivation (Blocher, 1997; Christophel, 1990, Gunawardena \& Zittle, 1997; Hackman \& Walker, 1990; Rourke et al., 2001). Only recently have studies begun to examine social presence as an important factor in the success of online learning (Picciano, 2002; Richardson \& Swan, 2003; Shih, 2004; Swan, 2001; Tu, 2000). In part, these studies inform us concerning the importance of the perception of social presence on interactions and the subjective measurement of learning outcomes in online courses. However, very few studies have examined social presence with empirical evidence in computer-mediated communications (Jiang \& Ting, 2000). In addition, few empirical studies have explored the relationship between social presence and persistence or retention in online learning.

Overall, the most salient issues to emerge from the literature on asynchronous learning are the need to increase and support active participation that will involve cognitive processes, such as active learning, collaborative construction of meaning, ideagenerating knowledge, and a sense of learning community through social presence (Gunawardena \& Zittle, 1997; Harasim, 1990; Kearsley \& Schneiderman, 1998). Williams, Paprock and Covington (1999) state, “As studies exploring concepts for establishing meaningful learning in distance education grow in number, so will our need in [the] understanding of distance education" (p. 11).

The limited amount of empirical research in the area of social presence, particularly as it relates to online learning, and the quality of perceived learning and satisfaction, supports the need for this study. Moreover, the lack of empirical research on whether the absence of social presence in online learning contributes to course attrition supports the need for further investigation in the area of social presence. Thus, the results 
of this study which proposes to examine the effects of social presence on a Web-based computer-mediated communication might increase our understanding of how learners' needs, experiences, and perspectives influence optimal educational environments and opportunities for online distance students.

\section{Problem Statement}

Social presence has emerged as an important factor in the field of distance learning (Gunawardena \& Zittle, 1997; Rourke et al., 2001). Of the empirical evidence that does exist, very little of it examines the social aspects and/or benefits of social presence in online learning, particularly in courses or programs of study that are totally online.

As learning is a social and human activity (Knowles, 1996) and not purely a technological process (Charp, 1998), changes in instructional method and medium are altering the roles of instructor and learner in online learning. Gunawardena and Zittle (1997) indicated the need to assess perception of social presence in computer-mediated communication by explaining that such environments tend to be more group-oriented instead of instructor-led. Because the unique perceptions of the instructor and learner are the basis of their individual role definition and may not always be congruent with one another, it is important to investigate what is valued in the online learning experience particularly from the perspective of the learner. Individual perceptions are an important consideration for designers and instructors when making decisions about the selection of technologies and pedagogies employed in course design, and there have been relatively few studies that support the claims of the perceived importance of social presence with empirical evidence (Jiang \& Ting, 2000). 
Two-way communication is crucial for a successful educational transaction to occur (Garrison, 1996). The ability to express and share ideas among learners and with the instructor promotes collaboration and deepens the learning experience. Ostensibly, deep and meaningful learning is the central goal of higher education and it is important to understand how the function of social presence can make the nature of online learning more interactive, appealing, engaging, and intrinsically rewarding leading to an increase in academic and social integration that results in increased persistence and course completion (Tinto, 1987).

These issues are some of the critical concerns that online providers and educators will need to tackle in order to address the requirements of effective online educational environments that best meet the needs of the learner. As technology in and of itself does not promote or ensure a successful learning experience for the learner, it is important for educators to know how to develop and plan learning opportunities and strategies in an online course that would be most effective in meeting the learners' needs and preferences regarding not only cognitive development, but also social presence and collaborative learning at a distance. Purpose of the Study

The purpose of this study is to explore learner perceptions and experiences of the learning process within the Web-based online learning medium in terms of social presence. More specifically, it examines the relationship among learners' perceptions of social presence in online courses and how it relates to perceived satisfaction of learning, whether course activities perceived as having high levels of social presence also have high levels of satisfaction and perceived quality of learning, and whether the learners' 
perception of social presence and satisfaction with learning affects their likelihood of enrolling in future online courses

Research Questions

The following research questions are used to explore the problem for this study within online courses that are delivered totally online.

1. What is the relationship between learners' perceptions of social presence and their perceived satisfaction with learning in online courses?

2. What is the relationship between learners' perceptions of social presence, satisfaction with learning experiences and quality of learning in online course activities, e.g., class discussions, group projects?

3. What is the relationship between the perceptions of social presence, satisfaction with learning, and the likelihood of future enrollment in online courses?

\section{Operational Definitions}

The following definitions are given to clarify terms used in this study to avoid misrepresentation.

Asynchronous- A communication method that does not require that the sender and receiver be present simultaneously at their computers for communication, such as email and discussion board. This is a time- and place-independent communication method.

Computer-mediated communication (CMC)- A process in which people produce, exchange, and perceive meaning within a variety of human contexts, verbally, 
nonverbally, textually, or iconically, using signs, symbols or cues via networked telecommunication systems. Online communication is a synonym for CMC.

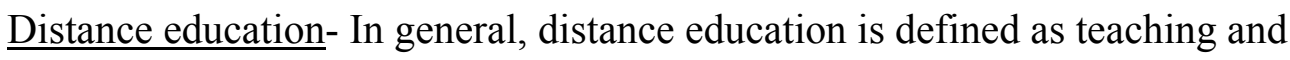
learning activities that occur with technology when the learners and the instructors are separated at a distance. It is an educational environment where (a) the instructor and student are separated during the main mode of instructional delivery so there is limited regular contact between instructor and students; (b) various media (print, audio, video, or computer) can be used to transmit content; (c) there is some provision for two-way communication in the educationalinstructional process; (d) people tend to receive instruction individually or in small groups (Keegan, 1988).

Immediacy- The extent to which selected communicative behaviors enhance physical or psychological closeness in interpersonal communication (Mehrabian, 1967). Such immediacy-producing behaviors include both verbal and nonverbal communication.

Interaction- The communication that occurs between the learner and content, the learner and instructor, and the learner and learner (Moore \& Kearsley, 1996). Intimacy- The sense of close connection one feels in a relationship (Argyle \& Dean, 1965).

Learning community- The term is often applied to online courses in which the instructor attempts to encourage class participation, discussion, and a high level of learner-learner interaction.

Online learning- The use of computer technology in delivering instruction primarily through the World Wide Web (WWW). Online courses typically use 
Web-based courseware or a combination of Web and email communication. It is also referred to as computer-mediated communication/instruction. Interaction between the instructors and learners and among the learners in this study is asynchronous.

Perceived learning- In this study, a quantitative and qualitative summary of students' answers to selected survey questions on learning experiences related to their specific online course. The concept is perceived learning, rather than simply learning, because it is measured via student self-reporting of their perceptions of the effectiveness of the instructional experiences.

Perceived satisfaction- In this study, a quantitative and qualitative summary of students' answers to selected survey questions on the state of being content and gratified with learning outcomes related to their specific course.

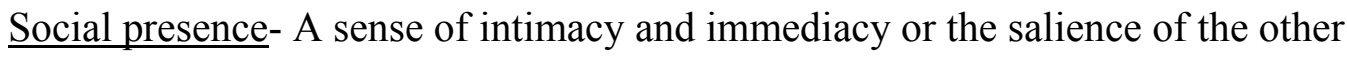
in a mediated communication leading to increased enjoyment, involvement, task performance, and socio-emotional interaction (Lombard \& Ditton, 1997; Short, Williams, \& Christie, 1976). In other words, a student's sense of belonging in a course or group and the ability to interact with others, although physical contact is not available (Picciano, 2002).

\section{Significance of the Study}

Online education for instructional delivery is growing rapidly as a field of practice. Web-based instruction is a relatively recent phenomenon and research in this area is in its infancy (Lombard \& Ditton, 1997). The need for research in asynchronous online education grows as the popularity and usage of online education becomes a new and emerging experience for students in higher education, thus making the need for 
educational designs and services that are of high quality, effective and personalized for the learners' specific needs in even more demand. Although recent attention has been given to online learning, it is important and necessary to devote more research efforts to examining the adult learner's perceptions related to online learning effectiveness (Phillips, Phillips, \& Zuniga, 2000).

New technological innovations always force one to evaluate how they may influence learning environments. Since their advent, computer technologies have been adapted not only to reinforce existing learning theories, but also to promote new approaches to learning (Pena-Shaff, Martin, \& Gay, 2001). Delivery of quality educational services and opportunities is becoming increasingly more complex as Webbased learning tools and support services continue to evolve. As recently as 2000, Kearsley reported that "wide-scale adoption of online education is just beginning and almost all of the research needed in this area has yet to be defined or conducted" (p. 56). Therefore, it is imperative that research on online teaching and learning expand so that we can examine the level of students' learning within this environment and determine how best to meet the needs of the learners.

Investigators early identified the unique nature of online communication including the benefits of high interactivity (Collins \& Berge, 1996). Although much effort is devoted to examining the use of technology to teach course content, an emerging area of importance in Web-based courses is how to enhance the student experience of learning and communicating online (McLoughlin \& Luca, 2002). Associated with this is the creation of social and supportive environments for learning when there is little faceto-face contact between learners and their instructors. 
The construct of social presence holds relevance for the use of computer-mediated communication in instructional delivery as well as perceived satisfaction and success in online learning (Gunawardena \& Zittle, 1997). Social presence therefore may be a significant factor in improving instructional effectiveness as it may help increase social interaction, encourage learning satisfaction, initiate in-depth discussions, and promote collaborative learning.

There is a need for further research in the area of socio-psychological perspectives in computer-mediated communication as the measures and research methods to date have been very few (Richardson \& Swan, 2003). Common themes that distance educators embrace are the need to make the learning experience personalized, affective, interactive, and positive (Hiltz, 1998). Spears and Lea (1992) argued that social presence is one of the most influential theoretical frameworks for analyzing computer-mediated communication. It has also been concluded that social presence exerts significant influence upon improving instructional effectiveness (Tu \& Corry, 2001); and, therefore, is of utmost importance to enhance learning in online learning. The amount and quality of social presence could play a strong role in the satisfaction and motivation level students derive from an online course as well as their perceived learning. With this concept in mind, Richardson and Swan (2003) stated, "further research is needed to determine the extent that perception of social presence influences student satisfaction, student motivation, and student cognitive and affective learning" (p. 18).

The construct of social presence was not designed to explain mediated communication between multiple individuals. Rather, Short et al. (1976) paired participants (dyads of one-to-one interactions) to evaluate and compare the quality of the media and their interactions. Although studies since have investigated the effects of 
social presence in computer conferencing (Gunawardena, 1995; Gunawardena \& Zittle, 1997), a useful direction for future research might be towards examining the importance of social presence with multiple individuals communicating together as well as participants' perceived value of social presence.

The body of distance education literature is lacking studies investigating the constructs of social presence and the value placed on it by distance learners involved in an asynchronous Web-based course. Moreover, the lack of empirical research on whether the absence of social presence in online learning contributes to course attrition supports the need for further investigation. As a result, it is necessary for research to be continued in these areas in order to assess student perceptions of social presence with its inherent attributes of interaction, intimacy, and immediacy and its value in relation to participation in asynchronous Web-based courses. This information could prove pertinent as to whether or not the existence of social presence in asynchronous courses is necessary for online learning and satisfaction. This insight could assist educators and instructional designers in planning, designing, organizing, managing, and delivering quality Webbased instruction in a manner that will improve student satisfaction and learning. Further research on learning in a Web-based environment is necessary to guide educators in delivering the best educational environment for learner achievement and satisfaction (Wynia, 2000).

Thus, the results of this study in examining the issues of social presence and its value in Web-based computer-mediated communication may help to add to the distance education literature and increase our understanding of how student needs, experiences, and perspectives may influence an optimal online learning community environment for online distance students. 


\section{CHAPTER II}

\section{LITERATURE REVIEW}

Chapter Two provides a review of the description of the salient literature relating to the following constructs pertinent to this study: an overview of online learning, its attributes, and concerns as it relates to higher education; the importance of interaction, social presence, and community relative to online learning and student satisfaction; the concepts of intimacy, immediacy and interactivity that have a mutual relationship with social presence; the theoretical constructs from socio-cognitive psychology that support the need for social presence in online learning; an overview of the importance and benefits of studying perceptions; and, finally, a discussion of the possible implications of social presence in an asynchronous Web-based learning environment.

\section{Online learning}

Online learning has been defined as any class that offers at least part if not all of its curriculum in the online course delivery mode as a transmission of information and/or communication via the Internet with instructors and students being connected regardless of time and place (Harasim, Hiltz, Teles, \& Turoff, 1995). This web-based instruction is known as a media-rich, online environment that allows people to interact with others in collaborative environments (Dede, 1995; Harasim, 1995), to gain access to remote multimedia databases through the Internet for active and resource-based learning (Hiltz, 
1994; Jung \& Leem; 1999), and to manage self-paced individual learning in a flexible way (Naidu, 1997; Reeves \& Reeves, 1997). Greater numbers of students than ever before are enrolled in distance education through enhanced college courses, collaboratives with other universities and colleges, and in some cases through completely virtual universities (Phipps, Wilman, \& Mersater, 1998). Online enrollments were expected to exceed $2,000,000$ (up from 500,000 ) across the $85 \%$ of two-and four-year colleges and universities offering courses online (American Federation of Teachers, 2001).

New advances in Internet-based technology have brought challenges and opportunities to education and training, in particular through online instruction. This type of instruction is perceived as a major breakthrough in teaching and learning because it facilitates the exchange of information and expertise while providing opportunities for all types of learners in distant or disadvantaged locations (Hill, 1997; Webster \& Hackey, 1997). Primary among the challenges is how to meet "the expectations and needs of both the instructor and the student and how to design online courses so they provide a satisfying and effective learning environment" (Johnson, Aragon, Shaik, \& Palma-Rivas, 2000, p. 31).

Studies have shown that distance learners are characterized by particular attributes (Gibson, 1996; Hardy \& Boaz, 1997; Wolcott, 1996). The majority of distance learning students are adults between the ages of twenty-five and fifty; married; employed fulltime, and have family responsibilities. Moreover, females comprise $60 \%$ of this population and males 40\% (Hardy \& Boaz, 1997; Wolcott, 1996). 
Distance learners are also characterized as autonomous and self-directed (Connick, 1999; Gibson, 1996). Self-directed learning has become a prominent feature of adult education theory and practice (Merriam \& Caffarella, 1999). Knowles's (1975; 1980) concept of andragogy included the association that as adults mature they become more independent and self-directing. Philip Candy (1991), in a work that is widely regarded as a comprehensive analysis and discussion of self-directed learning, has constructed a conceptual framework for understanding self-directed learning as both a goal and a process which embraces four distinct phenomena: personal autonomy, selfmanagement, learner control, and autodidaxy- an approach to learning that takes place outside of institutions such as online learning. However, Imel (1988) adds that the learning experiences for most students have only been in structured and teacher-centered learning environments. As a result, these types of students may need guidance and assistance in accepting responsibilities for their own learning.

The literature of adult education emphasizes the characteristics of learners and the process of learning, and provides insights into methods that are appropriate for facilitating learning (Merriam \& Caffarella, 1999). Since distance learners' characteristics, needs, and learning preferences differ, it is important for those designing and teaching at a distance to consider the appropriate instructional methods that may impact learning in this unique environment (Moore \& Kearsley, 1996). One assumption of self-directed learning is that learning is independent of a facilitator or some outside resource. However, Garrison (1997) views self-directed learning from a collaborative constructivist perspective. He believes that students are motivated to assume personal responsibility and collaborate to construct their own meaning through critical thinking. 
This does not mean students are independent and isolated learners. Facilitators should provide the standards, support and direction necessary for a successful educational outcome. Candy (1991) noted that knowledge is socially constructed and that learning is a social process. "Educators should not, in advocating self-direction in learning, lose sight of the fact that contact with other people is essential to most forms of learning. Self-direction does not necessarily imply solitary learning" (Candy, 1991, p. 367).

Distance education using the Internet is different in many aspects from other means of instruction. Online distance education is characterized by the absence of immediate feedback, both audio and visual, the absence of visual cues, and the nature of computer-mediated communication that can be both isolating and interactive (Harasim, 1990). Lack of nonverbal cues can create misunderstanding and some students are not comfortable with the different kind of interaction that takes place electronically as compared to what takes place in traditional classrooms (Kerka, 1996). Reliance on learner initiative can be a drawback for those who prefer more structure. Social isolation and the lack of nonverbal cues can hinder communication, interaction and learning (Connolly, Jesup, \& Valacich, 1990; Hiltz, 1986; Sproull \& Kiesler, 1991).

In the technological era of web-based learning and online course offerings, there is an increasing need to understand the social contexts of learning that are created by this unique type of learning environment. It is known from research on learning processes in a traditional face-to-face learning environment that development of social climate is important to make students feel like they are a part of the learning community, thus contributing to students' motivation, involvement, learning outcomes, and contentment (Wegerif, 1998). Active approaches to effective learning emphasize learning as a social 
process that takes place through communication and interaction with others (Hiltz et al., 2000). Social interaction is claimed to be important in technology-mediated learning situations (Harasim, 1995) because of an absence of non-verbal cues and text-on-screen that provides a limited means of rich dialogue. Recent studies conducted by Jones (1998), Baym (1998), Agree (1998), Gunawardena (1995), Harasim (1993), Rheingold (1993), and Warschauer (1999) have emphasized the need for further research into the context of Web-based environments as they impact learning and communication.

Early distance education models were developed around the axes of autonomy, control, responsibility, and dependence/independence (Daniel \& Marquis, 1979; Garrison, 1989; Moore, 1989) as researchers attempted to define relationships among learner, instructor, and content when distance was an intervening factor. The discourse included studies of measurement that ascertained if learning was indeed occurring in the distance format and if it was as effective as instructional delivery in face-to-face learning environments (Russell, 1999).

Several studies specific to online learning have been reported to have significantly more effective outcomes than traditional-based courses (Russell, 2002), yet other studies continue to report no significant difference (Johnson et al., 2000). The majority of the research in distance education has focused on this continuous debate while limited research exists relative to outcomes such as student perceived learning and satisfaction with online course delivery.

As online learning was approached as a variant of classroom learning, the most effective way of investigating this delivery method was through an examination of its characteristics. According to Harasim (1990), online learning has five attributes that 
together set it apart from other educational environments: place-independent

communication; time-independent communication; text-based communication; many-tomany communication; and computer-mediated learning; all of which "create a unique social climate that impacts interactions and group dynamics online" (p. 43). It is also important to note that, while online education provides for many-to-many communication, it also allows for one-to-many and one-to-one communication as is needed. For example, online learning allows for instructor and student to communicate apart from the group when and if a situation warrants it, such as when discussing assignments and course grades.

Place-independent communication allows participants to work together regardless of geographic proximity, enabling participants to access and collaborate with instructors and peers anywhere. This feature also empowers learners who are unable to access traditional place-bound education, whether their reasons are geographic isolation, physical impairments, work and family responsibilities, or personal circumstances. Conversely, this physical separation also has a tendency to give rise to a feeling of disconnection (Kerka, 1996), isolation, and lack of personal attention resulting in dissatisfaction with the learning experience (Besser \& Donahue, 1996; Twigg, 1997).

The attributes of time-independence, text-based communication is a double-edged sword for the learner. It may facilitate participation and allow the participants the ability to be reflective and to compose thoughtful rather than spontaneous responses (Edelson, 1998; Whitworth, 1998), as well as have a democratizing effect that prevents discussions from being dominated by a few articulate or verbose speakers (Saye, 1997). Although in one study (Cravner \& Michael, 1998) it was discovered that in classes which met both 
face-to-face and online, students who occupy face-to-face discussion also dominate the computer-mediated discussion. Another advantage of online communication is that it gives increased opportunities for participant input thereby enhancing the quality of decision-making (Rice, 1984).

On the negative side, however, there is also a sense that the inherent delays in asynchronous communication may operate against the development of a dynamic and interactive online discussion. Therefore, it may leave students feeling remote, detached, and isolated and discourage them from participating (Connolly et al., 1990; Hiltz, 1986; Sproull \& Kiesler, 1991). The pace of the coursework and the time commitment required of an online course demands motivation, self-reliance and time management skills as well (Ben-Jacob, 1998).

Time and place-independence may become unmanageable responsibilities rather than features for the self-disciplined and self-directed learning that is generally required in an online learning environment (McIsaac \& Gunawardena, 1996). Knowles (1973) advised that teachers consider the time constraints and pressures placed on the adult learners' studies by their different roles and responsibilities. Students may underestimate the amount of time required to succeed in online courses (Kember, 1989). Students who are not self-directed may not be able to create an effective timetable for interaction with online course elements to complete a course in the expected time. Some students may perceive online courses to be less rigorous, perhaps assuming that the amount of content and the demands on their time is small relative to traditional, face-to-face courses (Smith \& Winking-Diaz, 2004). 
In addition, learning-style preferences and personality may result in a greater degree of comfort in the online environment almost immediately, whereas others struggle with and in some cases never accept it (Tu \& Corry, 2001). The text-based communication may create a sense of detachment and a feeling of anonymity brought about by the lack of visual and auditory cues and the reliance on textual communication. As a result, students may feel no connection with their peers and thus feel no compulsion to go beyond the minimum participation required in the online course (Hill, 1997).

One attribute of computer-mediated communication is the random access to a permanent record of conference discussions (Edelson, 1998; Kaye, 1987). This feature may be viewed positively as enhancing participation by allowing students to read selectively and reread and review when necessary. Conversely, it may serve to discourage more active participation because students feel they are able to access all the information by reading other students' contributions. This is known as "lurking" or "vicarious interaction"; this is a familiar concept in computer-mediated communication as one who only watches, choosing not to actively participate in the discussion (Althaus, 1997; Romiszowski \& Ravitz, 1997). Another negative manifestation of the permanent record experienced by some students is information overload. As the course progresses, the record gets longer and the ability to deal with it becomes more of a problem for students, particularly those who do not have the self-discipline or time to participate regularly (Edelson, 1998).

As noted, online communication has several educational benefits. It has the potential to increase the students' access to educational opportunities due to the convenience of anytime, anywhere accessibility, as well as increase their responsibility, 
initiative, participation, discussion, and peer interaction, providing greater learning (Harasim, 1990; Jiang, 1998). In short, it prepares more independent learners (Althaus, 1997; Brandon \& Hollinghead, 1999). However, it is not without its disadvantages in challenging learners to effectively communicate and collaborate in primarily a text-based medium. In summary, online learning may not be better or worse than in a face-to-face classroom, it is simply different. One is not necessarily a higher quality of interaction and instruction, but both have their strengths and shortcomings (Garrison, 1993).

\section{Interaction}

Among the many features of Web-based instruction and computer-mediated communication, expanded interactivity is especially important in overcoming one of the shortcomings of traditional distance education, that is, lack of interpersonal interaction (Jung, Lim, Choi, \& Leem, 1998). The delivery medium allows people to interact with each other, with instructors or outside experts, and with multimedia resources. Asynchronous communication features such as email, listservs and computer conferencing allow for time- and place-independent interactions.

Educational and communication research identifies peer interaction among students as a critical component in learning and cognitive development (Anderson, 2002; Holmberg, 1983; Moore, 1993; Vygotsky, 1978). Such theorists characterize learning as an interactive group process in which the learners actively construct knowledge and then build upon that knowledge through the exchange of ideas with others and the responses/feedback of others (Harasim, 1990; Vygotsky, 1978). While learners interact while working in a learning activity, they bring their own framework and perspectives to the activity. They can see a problem from different perspectives, and are able to 
negotiate and generate meanings and solutions through shared understanding. A unique feature of online education is its capabilities to support this interactive group process.

In online learning, interaction with people is at a distance and is generally mediated through discussion tools such as email, bulletin boards, threaded-discussion forums, and computer conferencing allowing for time-and place-independent interactions. The functionality and ease of use of these tools facilitates interaction or makes it more difficult, depending on one's experience with these tools (Hillman, Willis, \& Gunawardena, 1994). If interaction with people is important and discussion is the primary venue for this interaction, we should understand why learners choose to (and not to) participate, to optimize decisions about design and facilitation of online learning environments.

Interaction has been described as "a defining characteristic of education" (Moore \& Kearsley, 1996, p. 128), as being "vitally important" (Moore, 1989, p. 6), as desirable with positive effects (Kearsley, 1995), as a critical component of the process of teaching and learning (Anderson, 2002), and as the primary goal of the educational process (Berge, 1999). Interaction is assumed to be a key aspect of effective learning and information exchange (Keegan, 1990), and "education at its most fundamental form" (Shale \& Garrison, 1990, p. 1). Irani (1998) found that as interaction increased, students' satisfaction for the course and learning outcomes improved as well.

Fulford and Zhang (1993) found that a high level of interaction among learners and between learners and instructors led to positive attitudes toward the course subject and higher achievement with instruction in a televised course. These researchers concluded that the learners' perception of interaction is a vital indicator of their 
satisfaction with the instructor. The level of interaction, according to Muirhead (2001), has an impact on the quality of computer-mediated instruction. Given its importance in the literature, it is ironic that there is an apparent lack of research about interaction (Jiang \& Ting, 2000; Kearsley, 1995).

Critics of online education have raised concerns about the level and quality of student interaction in classes offered over the Internet (Stross, 2001). It has been noted that interaction is missing in many online courses (El-Tigi \& Branch, 1997), possibly caused in part by instructors' inexperience with the medium (Wolcott, 1996), the lack of instructional design (Jonassen, Davidson, Collins, Campbell, \& Haag, 1995), or unclear definitions of what interaction is and is not (Roblyer \& Ekhaml, 2000). Merrill (2002) notes that courses which are primarily post, tell, and ask in nature are more informational than instructional and provide little value to learning.

It is often assumed by instructional course designers and instructors that increases in the amount of interaction will increase the quality of communication and naturally improve the quality of instruction (Gilbert \& Moore, 1998). Flanders (1970) claimed that increased interaction improved student achievement and attitudes toward learning in the traditional classroom setting with the most salient interaction being person-to-person and least salient when the teacher addressed the entire class. This same interaction of personto-person is essential in online learning. "Key to the learning process is the interactions among students themselves, the interactions between faculty and students, and the collaboration in learning that results from these interactions" (Paloff \& Pratt, 1999, p. 5). However, students have rated face-to-face courses higher on interaction, content participation, and faculty preparation (Lauzon, 1992; Ryan, Carlton, \& Ali, 1999). 
For example, an exploration study into computer conferencing was conducted by Lauzon (1992) to investigate student perceptions of computer conferencing. The results presented several views of learning and also revealed that students did not feel that computer conferencing was as good as face-to-face interaction. In addition, Berge (1999) noted that technologies most often used today are oriented toward the learners' interaction with the content alone, with little interaction between the learner and the instructor, and presumably less between the learners themselves.

Several studies have tried to clarify the concept of interaction in distance education (Bates, 1995; Moore, 1989; Moore \& Kearsley, 1996; Paulsen, 1995). Most references to the concept of interaction in the distance education literature cite three types of interaction as identified by Moore (1989). These are learner-content, learnerinstructor, and learner-learner interaction that can occur either synchronously or asynchronously. Moore described these three types of interaction as follows:

Learner-content interaction is the interaction between the learner and the content. Learner-instructor is the interaction between the learner and the subject matter expert (e.g., teacher), or some other expert acting as the instructor. Learnerlearner interaction is the interaction between one learner and other learners, individual, or within group settings, with or without real-time presence of an instructor (p.3).

Moore further recommends that distance educators use the expertise of media experts and communication specialists to assist with selecting communication media that will effectively enable each of the three types of interaction.

Hillman (1999) added a fourth type of interaction termed learner-interface. Learner-interface interaction is the interaction between the learner and the technology required for interacting with the teacher, students, and content. Hillman maintained that learner-interface interaction is as crucial as the other three types of interaction described 
by Moore (1989). Furthermore, it is important to demonstrate to students how to interact with the technology, such as threaded-discussion or submission of assignments as attachments to email. Learners who do not have the required skills to use the communication tools spend a lot of time learning how to interact with the technology and, as a result, can affect their time to concentrate on interactions with the instructor, other students, and course content (Hillman, 1999).

Both Moore (1989) and Hillman (1999) emphasized the importance of including strategies for successfully implementing these four types of interactions when designing a distance education course. They recommend designing activities that allow learners an opportunity to interact productively that could contribute to frequency of interaction and learner satisfaction.

Interaction is a key factor in distance education and is an important component of a successful instructional program. Whether students are interacting face-to-face or at a distance, their success may be a result of well-designed instructional strategies that take into consideration the factors that will promote interaction and enhance users' perceptions of satisfaction of their learning environment (Moore \& Kearsley, 1996). Hiltz (1986) assessed computer conferencing as an educational medium and concluded that the effectiveness for online learning "rests in whether students do take a more active part in the learning process and take advantage of the potential for more interaction with the professor and other students, despite the absence of nonverbal cues to facilitate this interaction" (p. 100). Absence of student interactions and participation could lead some students to feel isolated from the learning community. 
For students studying in an online course, interaction with other students and educators can often be an exercise in frustration. Asynchronous communication may not give the immediacy of interaction that is required for successful interaction and the inability to interact freely with other students may exacerbate feelings of aloneness and provide a less than ideal environment for successful study (McInnerney \& Roberts, 2004). This isolation could influence a student's attitude to online learning and possibly cause students undertaking online education to reconsider their enrollment (Daugherty \& Funke, 1998).

Garrison (1990) argues that the quality and integrity of the educational process depends upon sustained communication and interactivity. Without this connection, the student feels isolated, procrastinates, and eventually drops out. For students to learn in a distance environment, they must be an active participant in the learning process. According to Chickering and Gamson (1991), frequent contact is the most important focus in student motivation and involvement.

Isolation or the feeling of aloneness that many students feel is the hardest symptom for educators to combat (Palloff \& Pratt, 1999). This feeling of isolation is not always generated simply because of geographical distance as even on-campus students undertaking an online course may experience a feeling of isolation from the rest of the course participants. Other factors cited in the distance education literature that may lead to feelings of isolation include technical problems, computer literacy and cost (Fyfe, 2000).

Participation and persistence in distance education and those factors that contribute to their occurrence continue to interest practitioners and researchers alike. The 
negative outcome of persistence is referred to as dropout, withdrawal or attrition. The rate of persistence is considered a significant indicator of the effectiveness of the distance education programs (Cookson, 1990) yet dropout rate for online learning is much higher than for traditional on-campus students (Zielinski, 2000). As Powell, Conway and Ross (1990) so aptly note, “The question of why some students successfully study through distance education and others do not is becoming increasingly important as distance education moves from a marginal to an integral role in the provision of postsecondary education" (p. 5).

The information about students that those who design and deliver distance education need to know is also important for administrators whose job it is to build and maintain enrollment. Distance educators and institutional administrators, as are traditional educators and administrators, are concerned with enrollment, persistence and satisfaction patterns. Although it may not be true in every case, it may be assumed that if learners are satisfied with online education then persistence rates will be high. Conversely, if the withdrawal rates are high then it may be assumed that learners are dissatisfied with online education.

Enhancing students' satisfaction with online education courses may be an adequate goal for developers of distance education because it will increase the likelihood that students would enroll again in another course. A review of the literature indicates that researchers and practitioners alike want to know whether students will stay enrolled in online course offerings and will enroll again in the future, and how to improve the satisfaction of students with the online education course offerings (Biner, Birk, Huffman, 
\& Dean, 1995; Eastmond, 1995; Fjortoft, 1996; Luk, 1998; Powell et al., 1990; Schieman \& Jones, 1992).

Daugherty and Funke (1998) indicate that the issue of isolation is an important criterion for student satisfaction with the Web-based online course. This feeling is often based on the physical separation between student and instructor and is one that educators may be able to ameliorate by creating a sense of social presence that often leads to greater interaction and a sense of community (Wegerif, 1998). Wegerif (1998) stated that interaction is a main factor behind creating a high feeling of social presence.

Several studies have examined factors influencing interaction in computer-based instruction. Anderson and Harris (1997) identified that socially-oriented factors contributed to the prediction of both uses of the online network and outcomes and suggested the importance of implementing strategies to promote social interaction among users and of enhancing perceptions of a user's social presence. Similarly, a study conducted by Gunawardena and Zittle (1997) revealed that social presence was a factor that contributed more than $60 \%$ of learner satisfaction with computer conferencing courses. Hornik and Johnson (2003) and Richardson and Swan (2003) investigated the role of social presence in computer-mediated environments and found that students who show high social presence perceptions not only affect outcomes of student performance and satisfaction with their courses, but also affect students' perceptions of satisfaction with the instructor.

Kanuka and Anderson (1998) found that social-cognitive processes among participants in the online forum included significant time engaged in social interchange. Naughton's (2002) observations of the interaction activities in a text-based online 
community indicated that informal discussions represent more than double the amount of interaction as do course-related discussions. In addition, Evard, Churchill, and Bly (2001), argued that informal social interaction provides foundations for the community and that, in turn, supports the development of effective collaborative course-related problem-solving, strengthens the community work idea, and reduces the feeling of isolation. McDonald and Gibson (1998) found that interpersonal issues remained prominent through an asynchronous computer conferencing course. Dziuban and Moskal (2001) found a significant correlation between the quality and quantity of interaction in online courses and learners' satisfaction in Web-based courses based on a questionnaire. Thus, increasing the quality of interaction and not just the quantity may be a critical factor for the success of online learning.

If interaction has a mutual relationship with socially-oriented factors and social presence that enhances the participants interacting with each other within a computermediated environment, then it is important to explore this interrelationship and the role social presence has in improving interpersonal communication and interactions among distance learners.

\section{Social Presence}

The construct which has come to be known as "social presence" is rooted in the work of Mehrabian (1969) on what he has termed "immediacy" which he defined as "those communication behaviors that enhance closeness to the nonverbal interaction with another" (p. 203). His work was followed up by a number of communication theorists including Short, Williams, and Christie (1976), who postulated that the inability of some 
communication media to project non-verbal communication would negatively affect interpersonal communication carried via such media.

Short et al. (1976) were the first to introduce the concept of social presence. They based this concept on previous research that addressed interpersonal communication between dyads-one-to-one communications. These authors defined social presence as the "degree of salience of the other person in the interaction and the consequent salience of the interpersonal relationships..." (Short et al., 1976, p. 65). Social presence, by this definition was initially hypothesized to be an attitude of the medium itself, and was called technological presence.

Other researchers (Gunawardena \& Zittle, 1997; Lombard \& Ditton, 1997) concur with Short et al. (1976) in that the qualities of the medium may have an effect on the degree of social presence, but have implied this to mean the degree to which individuals perceived each other as "real" within the context of their mediated communication. This perception varied among users and should be viewed as a subjective quality, depending on the objective quality of the medium (Walther, 1982). This suggests that social presence should be viewed as the users' subjective perceptions that depend on the objective quality of the medium.

As social presence is deemed to be a dynamic variable, people discern different amounts of social presence in various types of media. This implies that the degree of social presence can be measured in a particular situation (Perse, Burton, Kovner, Lears, $\&$ Sen, 1992; Walther, 1992). The users are asked to judge the degree of social presence.

Short et al. (1976) maintained that the measurement of social presence is subjective and involves an attitudinal dimension of the user towards the medium. The 
method of measuring was through a semantic differential technique questionnaire and used a series of bipolar scales: sociable/unsociable, personal/impersonal, sensitive/insensitive, and warm/cold (Short et al., 1976). It was based on Osgood's (1957) semantic differential technique. The findings from comparison studies on the types of media conducted by Short et al. (1976) claimed that the capability of the medium to transmit verbal and nonverbal cues or behaviors contributes to the degree of social presence of the medium. Nonverbal visual cues include communication other than speech that is conveyed through gestures, facial expressions, gaze, posture, and other interpersonal behaviors. Nonverbal communication can play a significant role in facilitating interaction. Facial expressions, gaze, and body language can reflect an individual's culture, personality, and mood and have a significant effect on heightening the quality of interaction.

Face-to-face communication was the most preferred mode of communication due to the number of social cues such as dress, gaze, posture, and eye contact that this media is capable of transmitting. Media perceived to have a higher degree of social presence were described as warm, personal, sensitive, and sociable. Conversely, media perceived to have a lower degree of social presence were described as cold, impersonal, insensitive, and unsociable.

In addition, the degree of social presence includes how well the media is able to conduct interpersonal interactions. Two types of interactions identified by Short et al. (1976) are task and social. Task-driven interaction is directed toward the completion of assigned tasks while social-interaction is directed toward relationships among learners. Task-driven interaction is under the direct control of the instructor and often takes the 
form of responses to instructor-generated discussion topics and peer assessments. As mediated communication becomes more task-oriented the interaction moves from informal to formal. This supposedly decreases the degree of social presence (Argyle \& Dean, 1965; Wiener \& Mehrabian, 1968).

In contrast, a socio-emotional driven interaction is largely self-generated. Socializing can take on many characteristics, from exchanging empathetic messages (McMahon, 1997) to self-disclosure (Cutler, 1996). Self-disclosure is personal information about an individual that is voluntarily revealed to another and is information that cannot be obtained from any other source (Adams, 2004). Self-disclosure plays a key role in the development and maintenance of trust and open relationships and, in turn, can provide the foundation for information sharing and the building of learning communities (Baird, 1977; Rawlins, 1992). As a result, a socially-oriented interaction supposedly increases the degree of social presence. Thus, the type of interpersonal interaction affects an individual's perception of the mediated communication and the attitude towards the medium.

For example, in one study a business letter was identified as having the lowest degree of social presence in comparison to face-to-face, telephone, closed-circuit television, and audio due to the inability to convey non-verbal and verbal cues and its preference for tasks low in interpersonal involvement (Argyle \& Dean, 1965; Wiener \& Mehrabian, 1968).

Current studies conducted on social presence include the traditional classroom and computer-mediated conferencing medium settings (Blocher, 1997; Gunawardena \& Zittle, 1997; Hackman \& Walker, 1990). The results from these studies revealed that 
students perceived differences between face-to-face and mediated communication and that the perceived media differences affect student satisfaction along with their perception of the mediated learning experience. Generally, students perceive face-to-face communication as having the highest degree of social presence. The literature strongly indicates both students' and instructors' desire for contact (Rezabeck, Meyers, \& Edwin, 1992). Although this study specifically focuses on the importance of student satisfaction toward an online learning environment, it is recognized that there is also a need to focus on instructor attitudes and specific factors- motivating and inhibiting- affecting participation in online, Web-based teaching (Northrup, 1997; Dillon \& Walsh, 1992).

$\mathrm{Tu}$ (2001) redefined social presence in computer mediated communication as "the degree of feeling, perception, and reaction to another intellectual entity in the CMC environment." He identified three dimensions of social presence that included social context (individual personalities, attitudes, motivations, and perceptions), online communication (attributes and applications of the language used online), and interactivity (activities in which the users engaged and communications styles they used). In this sense, social presence is interpreted as the consequent appreciation of an interpersonal relationship which is shaped by social context, online communication and interactivity.

Nonverbal communication has its most direct instructional impact on the affective domain of learning, which is concerned with student attitudes, values, beliefs, appreciations, and interests. Factor-analytic studies indicated that positive affect is communicated through a cluster of nonverbal behaviors, labeled immediacy behaviors (Anderson, 1979). Immediacy behaviors increase sensory closeness, communicate 
feelings of warmth and support, and engender feelings of interpersonal attraction (Anderson, 1979).

It is the nonverbal cues to communication that many feel are lost with computermediated communications. The early research on social presence on computer-mediated communication (Hiltz, 1986; Walther \& Burgoon, 1992) concluded that this medium was unable to provide social context cues and was, therefore, considered to possess limited social presence because it was perceived as being an impersonal medium. Tuckey (1993) reinforced the notion that the use of computers for communication also means that the social context cues associated with communication such as nonverbal cues may take some of the emotion and social aspects out of the communication process thus leaving the "exchange cold and unsociable" (p. 64).

In practice, however, participants may well be able to compensate for this lack of nonverbal communications. For example, Walther and Burgoon (1992) in their research on relational communication in computer conferencing found that the differences in communication between computer-mediated communication and face-to-face learners tends to be eliminated over time if interaction time is not constrained and learners become more familiar with one another. "Although initial interactions among unacquainted others in CMC may be relatively low in immediacy/affection, interactants may increase this dimension over time" (p. 58).

Likewise, Gunawardena (1995) found that although the computer conference medium is low in nonverbal cues, "students create social presence through the computer conferencing medium by projecting themselves online" (p. 9). Gunawardena (1995) suggested that by successfully "inculturating” themselves within computer-mediated 
communication, learners promote their level of social presence and allow themselves an opportunity for greater participation.

Equilibrium theory (Gunawardena, 1995) might also play a role in the absence of nonverbal communication. This theory states that people will use other means to achieve that which they perceive to be absent from an instructional environment. For example, in text-based environments, the ability to utilize emoticons and paralanguage, such as combinations of punctuation to convey a smile $\odot$, in illustrating various emotions while communicating becomes very important in an online learning environment (Gunawardena, 1995). Gunawardena and Zittle (1997) found that participants in a computer conference enhanced their socio-emotional experience through the use of emoticons to express nonverbal cues.

Danchak, Walther, and Swan (2001) suggest that social presence in mediated environments is a function of both the affective channels of communication, such as paralanguage, and the immediacy behavior of the participants. This is the ability of participants to project themselves socially and affectively through responses that contain personal expressions of emotion, feelings, humor, self-disclosure beliefs, and values. They argue that these behaviors will evoke a kind of equilibrium of social presence with which the participants are comfortable. That is, students' perceptions of social presence will reflect on their communication behaviors.

Swan's (2001) content analysis of discussions in an online graduate course supports the equilibrium theory of social presence. In this analysis, a total of 1,336 in 235 postings or an average of 6 verbal immediacy indicators per response were found throughout the entire discussion postings. The results provide evidence that participants 
in this course compensated for the lack of nonverbal cues by employing more immediacy behaviors in the written form (Danchak et al., 2001). Overall, the findings imply the need for designing computer-mediated environments where attention is paid to techniques that enhance social presence (Gunawardena \& Zittle, 1997).

The literature on social presence indicates that an interrelationship exists between social presence and communication cues, both verbal and nonverbal (Moore \& Kearsley, 1996). Studies conducted on the effects of social presence in computer-mediated conferencing have indicated that it is possible to relay certain socio-emotional behaviors associated with intimacy and immediacy using the text-based features in computermediated technologies (Danchak et al., 2001; Gunawardena \& Zittle, 1997; McIsaac, Blocher, Mahes, \& Vrasidas, 1999). In spite of the characteristics of the medium, student perceptions of the social and human qualities of computer-mediated communication will depend on the social presence created by the instructor and the participants of the online community that is formed involving intimacy and immediacy behaviors (Gunawardena, 1995; Gunawardena \& Zittle, 1997). The relationship between intimacy, immediacy, and social presence is therefore worth examining.

Intimacy and Immediacy

Gunawardena and Zittle (1997) suggest intimacy and immediacy are two concepts associated with social presence in which intimacy is dependent on nonverbal factors, including physical distance, eye contact, and smiling. Immediacy is a "measure of the psychological distance that a communicator puts between himself and the object of his/her communication" (Gunawardena \& Zittle, 1997, p. 9). This may include behaviors such a vocal inflections, gesturing, physical proximity, and spending time with someone. 
To be clear, indicators of intimacy include familiarity, empathy, concern, and trust (Heath \& Bryant, 1992). Indicators of immediacy include ability to exchange information rapidly, personalized exchanges, use of inclusive language such as "we" or "us", and individualized feedback (Christophel, 1990; Gorham, 1986; Wiener \& Mehrabian, 1969). Immediacy behaviors are essential to create and maintain intimacy.

The levels of intimacy that people adopt are derived from cultural norms (Argyle \& Dean, 1965) and from a need for affiliation (Walther, 1992). The depth of selfdisclosure between individuals in a relationship determines the level of intimacy. A greater level of self-disclosure characterizes a relationship with a high level of trust (intimacy). According to Cutler (1996), "the more one discloses personal information, the more others will reciprocate, and the more individuals know about each other, the more likely they are to establish trust, seek support, and thus find satisfaction" (p. 326). Thus, increased disclosure of personal information can help strengthen a learning community.

Both proximity and physical closeness have been associated with intimacy (Argyle \& Dean, 1965). Accordingly, an individual permits another to enter their personal space based on the degree of intimacy present. A higher degree of intimacy enhances familiarity, self-disclosure, and trust, whereas, a lower degree of intimacy provides the opposite. Voice tone, pauses, and inflection of speech can also convey intimacy and project a higher sense of presence than text alone.

Another concept associated with establishing closeness with others by using words and language is immediacy. A person can convey the idea of immediacy both verbally (praise, humor, addressing students by name) and nonverbally (facial 
expressions, gestures). In other words, an individual can transmit socio-emotional behaviors that can convey closeness (immediacy) or distance (non-immediacy) by speech and nonverbal behaviors (Wiener \& Mehrabian, 1968).

Kearney, Plax, and Wendt-Wasco (1985), Gorham (1998), and Christophel (1980) provide some of the early descriptions of the concept of social presence from an instructional communication perspective, defining it as "teacher immediacy" in the classroom. Findings from immediacy studies conducted in educational settings (Christophel, 1990; Gorham, 1988; Hackman \& Walker, 1990; Moore, Masterson, Christophel, \& Shea, 1996) suggested that students view more favorably teachers who employ verbal and nonverbal immediacy behaviors. These two types of immediacy behaviors convey closeness.

For example, Moore et al., (1996) conducted research examining the relationship between teacher immediacy behaviors such as eye contact, immediate feedback, praise, and student satisfaction. This study investigated the relationship between college students' perceptions of teacher immediacy behaviors and students' ratings of instruction. Significant positive correlations were found between teacher immediacy behaviors and students' ratings of instruction. More specifically, verbal and nonverbal immediacy behaviors were strong predictors of students' ratings of faculty/student interactions and lectures and communication. In addition, post hoc analyses indicated that additional variables, such as class size, subject area, and students' expected grade influenced immediacy scores and student ratings. These findings demonstrated the importance of incorporating teacher immediacy behaviors when assessing teacher effectiveness. If teacher immediacy behaviors contribute to student learning (cognitive, behavioral, and/or 
affective) then teachers should incorporate and monitor these types of activities into their classroom behaviors (Moore et al., 1996). The results from these studies indicated that intimacy and immediacy are positive indicators of student learning outcomes and student satisfaction, and that they contribute to social presence.

For example, highly immediate behaviors have been associated with attitudinal changes, such as increases in student motivation to study and learn (Christophel, 1990; Christophel \& Gorham, 1995) and student satisfaction (Hackman \& Walker, 1990; Moore et al., 1996; Richardson \& Swan, 2003) where students' ratings of instruction were positively correlated with students' perceptions of teacher immediacy behaviors. Students who perceived more frequent verbal and nonverbal immediacy behaviors in their teachers were more likely to give higher ratings to the overall quality of instruction and value the course.

Researchers have also investigated the effects of teacher immediacy on affective learning (Kearney et al., 1985) and cognitive learning (Kelley \& Gorham, 1988; Sanders \& Wiseman, 1990). Kearney et al. (1985) examined the salience of immediacy and teacher nonverbal immediacy as potential indicators of students' affective learning across different types of course content. The course content in their study ranged on a continuum from P to T-type courses. P-type courses were concerned primarily with people-oriented content (psychology, sociology) while T-type courses were product or task-oriented (accounting). The results of their study and previous research on nonverbal teacher immediacy behaviors suggest that teacher immediacy is critical for particular affective learning outcomes in both types of courses. The researchers claim that due to the nature of variability in the course types they investigated in addition to the types of 
classes previously investigated (Anderson, 1979), their findings support the existence of an immediacy/affect relationship across learning environments (Kearney et al., 1985).

In an attempt to examine the cognitive effects of teacher immediacy behaviors, Kelley \& Gorham (1988) separated out the effects of teacher immediacy behaviors on cognitive learning from their effects on affective learning. Short-term recall functioned as the experimental variable for cognitive learning. Immediacy was based on eye contact and the physical immediacy of the instructor. The results of this study suggested a positive relationship between immediacy and cognitive learning at the short-term recall level.

Sanders and Wiseman (1990) expanded this relationship to include behavioral and cognitive learning. These authors operationally defined cognitive learning as how much students thought they had learned in a course. The authors defined behavioral learning as the likelihood that students would actually attempt to use the behaviors, practices, or theories studied in the course. Positive correlations between both nonverbal and verbal teacher immediacy behaviors and student affective, behavioral, and cognitive learning were significant.

Gunawardena (1995) argues that immediacy enhances social presence. Thus, the degree of presence is not simply an attribute of the medium. Increasing the intensity of immediacy can enhance social presence. For example, Gunawardena's (1995) study of computer conferencing suggested that it is the skilled use of the instructor's interaction techniques by initiating online conversations with introductions and salutations that will impact the students' perceptions of social presence. Social interaction augments the relationship between learner-instructor and learner-learner in the learning environment. 
Consequently, instructors should develop interaction skills that create a sense of social presence.

Lombard and Ditton (1997) claimed that it is possible to achieve real immediacy within mediated interactions and conversation. Ditton further claims that communication media has the potential for conveying immediacy in written form. Immediacy, as it relates to written form (text), has relevancy in distance learning environments due to the use of text-based transactions in mediated communication. In a mediated learning context, the use of immediacy strategies such as calling students by name, praising students for work well done, showing concern, providing immediate feedback, and referring to the learning community as "we" (Gorham, 1988; Wiener \& Mehrabian, 1968) may positively affect the learner's perception of the presence of others and foster a sense of feeling included in the learning community. Others have agreed that this is essential to minimizing psychological distance and to improving the sense of a learning community (Gunawardena, Lowe, \& Anderson, 1997; Sherry, 1996; Wolcott, 1996).

These immediacy studies suggest that social presence is a critical factor that impacts interactions in online learning environments. They also suggest that greater perceptions of social presence tend to be linked with greater perceived learning, course satisfaction, and satisfaction with the instructor (Gorham, 1988; Gunawardena, 1995; Gunawardena \& Zittle, 1997; Picciano, 2002; Richardson \& Swan, 2003; Walther, 1992). Since researchers suggest that a sense of presence provokes meaningful interactions that contribute to collaboration and construction of knowledge or a community of learning (Rovai, 2002), it may be that a students' sense of presence actually improves their 
perception of learning and motivates their learning. What is not clear from the current research is how social presence influences learning and retention in an online course.

\section{Relationship between Social Presence, Interaction, Intimacy, and Immediacy}

The concepts of interactivity, intimacy and immediacy have a mutual relationship with social presence and make up the construct that comprises social presence. If a high degree of social presence exists then high degrees of intimacy, immediacy, and interactivity also exist (Gunawardena \& Zittle, 1997; Hackman \& Walker, 1990). Social presence is dependent on the degree of intimacy and immediacy, and these two concepts are contingent on the quality of the interactivity (Mehrabian, 1969; Short et al.,1976). Likewise, the level of intimacy is dependent on the level of immediacy, and the degree of social presence correlates with all of these variables.

According to the communication literature, there are indicators associated with each of the three concepts (Argyle \& Dean, 1965; Gorham, 1988; Heath \& Bryant, 1992; Mehrabian, 1969; Moore, 1989; Short et al., 1976; Wagner, 1994, 1997; Wiener \& Mehrabian, 1969) that enhance the degree of social presence among the participants within a mediated environment. For intimacy, these include trust, association, familiarity, self-disclosure, and affiliation. Close acquaintances, associations, concern for others, and familiarity are indicators of high levels of intimacy (Heath \& Bryant, 1992). For immediacy, these include personalized exchanges, use of inclusive language such as "we" or "us", and individualized feedback (Christophel, 1990; Gorham, 1986; Wiener \& Mehrabian, 1969). The indicators for interactivity depend on the quality of interactions between learner-instructor, learner-learner, and learner-content (Moore, 1989). 
Given that technology facilitates the communication and interaction in mediated environments, it is important to examine the degree of intimacy, immediacy and interactivity that exists in this environment to enhance social presence and foster a sense of a learning community.

\section{Community}

The feeling of isolation and psychological distance is overcome when students join together in a community of learners who support one another (Eastmond, 1995). The process of forming a community of learners is an important issue in distance learning because it can affect student satisfaction, retention, and performance (Misanchuk, Anderson, Craner, Eddy, \& Smith, 2000; Neo, 2003).

The notion of using community as an ideal place for learning in education builds on the changes in paradigm from teaching to learning (Barr \& Tagg, 1995). Being a part of a learning community can generate a positive learning experience for students as engaging in interactions can promote problem-solving and critical thinking (Saiedian, 1993). Mason and Kaye (1990) described computer conferencing as defining a new paradigm in distance education that can provide enhanced opportunities for dialogue, debate, and conversational learning and the potential for a real sense of community with access to other students' experiences and opinions. They predicted that computermediated communication would develop a potentially new type of learning community that would provide a space for collective thinking and access to peers for socializing and communication.

Using computer-mediated communication with distance learners has been viewed as having the potential to change the nature of distance education, with an ideal of "a 
collaborative, respectful interdependence where the student takes responsibility for personal meaning as well as creating mutual understanding in a learning community" (Garrison, 1993, p. 17). Garrison (1995) approaches online learning from a transactional constructivist perspective that constructs and validates "meaningful and worthwhile knowledge...through both individual reflection and social discourse" (p. 138). Harasim (1990) described the greatest strength of online education as its ability to facilitate interaction and saw the strength of CMC in group activity. The social, affective, and cognitive benefits of peer interaction and collaboration, which had previously been possible only in face-to-face situations, were now possible with the mediation of computer communication.

These researchers and writers have described the potential of the medium as an interactive environment that would enable collaborative group learning and would change the nature of distance education from an autonomous, isolated experience to a potentially social constructivist environment. Distance educators, based on their distance teaching experiences, recommend strategies considered effective to promoting a community of learners.

For example, Wagner (1997) suggests first considering the course goals and objectives in order to effectively design an interactive learning community. Strategies for design consideration as proposed by Wagner (1997) include group work, discussion forums, seminars, and problem-solving. Aside from applying these strategies, Wagner (1997) suggests maintaining the learners' involvement, encouraging student cooperation and collaboration, providing timely feedback, and implementing various instructional strategies in consideration of different learner styles. Walker and Hackman (1991) 
recommends that teachers use immediacy behaviors, such as using the pronoun "we" or "us" to convey inclusion of all members of the learning community.

Harasim (1996) claims that, in any educational setting, social communication is essential in forging social bonds that have important socio-affective and cognitive benefits. To accomplish this, Palloff and Pratt (1999) suggest that course introductions should include social activities, which permit informal interactions to ensure students are acquainted and have opportunities to establish rapport with each other.

Rourke et al. (2001) define social presence as the ability of learners to project themselves socially and affectively into what they call a community of inquiry. This definition tends to place more responsibility on the ability of learners while Gunawardena (1995) places the onus on the instructor to create a sense of community online. These are not necessarily at odds, but point to the interdependency of all participants in an online learning environment.

In order to foster a community or sense of belonging within the group, the online teacher's role must change to that of a facilitator. The more traditional role of the teacher as the complete mediator of an instructional experience must change. The teacher's primary role should be one of facilitator and supporter rather than dispenser of information (Harasim, 1995; Hiltz, 1994; Gunawardena, 1992; Jonassen, 1995). Learning and development then becomes a social activity and it is during this process that the teacher acts as a facilitator. The facilitator is responsible for creating a social environment. A trusting, social environment permits students to feel comfortable with their peers and to feel a sense of involvement. This is considered important to building a “sense of community" (Gunawardena, 1992; Palloff \& Pratt, 1999). In order to 
encourage interaction and collaborative learning, it is important that teachers/facilitators of computer-mediated communication promote the creation of conducive learning environments. "Students can be trained to create social presence in a text-based medium and build a sense of community" (Gunawardena, 1995, p. 163).

Students also need to assume responsibility for creating positive learning environments for themselves and others. Creating social presence is not the sole responsibility of the instructor. Suggestions by distance educators to students for facilitating social presence in the online environment include participating on a regular basis in discussions, promptly responding to email, sharing personal stories and experiences as they relate to the topic, using emoticons as nonverbal social cues, and addressing others by name (Aragon, 2003). Norman (1998) argued that interaction strengthens learner's awareness, attention and involvement, which in turn, increases their level of the feeling of social presence.

It is paradoxical that on one hand technology solves the problem of geographic isolation, yet on the other, can also be the cause of psychological and social isolation. In the years that the Internet has come into more widespread use for higher education program delivery, online collaborative learning has become more commonly accepted as an effective strategy that is now made possible by the technology. To maximize this technology to enhance learning, it is the instructor's responsibility to ensure students get connected and become part of the online learning community, and yet, it is also the responsibility of the student to participate as members.

In text-based online discussion an additional benefit of social presence, according to Rourke et al. (2001), is its ability to encourage, support, and sustain cognitive learning 
objectives by making group interaction engaging and rewarding, thereby promoting course completion and continued study. Rourke et al. (2001) explained that the importance of the feeling of social presence in the collaborative learning environment is obtained from its ability to prompt, maintain, and support cognitive and affective learning objectives by making group interactions, interesting, engaging, attractive, and rewarding. In considering the social interaction dimension of online learning communities, it is important to consider the development of socio-cognitive environments. Socio-cultural and constructivist theories have begun to be accepted as theoretical perspectives for explaining the effectiveness of collaborative learning in an online environment to the extent that Kanuka and Anderson (1998) claim socio-cultural theory is "currently the most accepted epistemological position associated with online learning" (p. 60). The constructivism philosophy provides the distance learning field with a theoretical justification for the study of interaction, particularly learner-learner, as it advocates collaborative learning as a process of knowledge construction on the part of individual students (Kanuka \& Anderson, 1998).

\section{Socio-cultural and Constructivist Theory}

Collaborative learning environments are reported to promote thinking and problem solving and allow students to perform at higher intellectual levels that lead toward deeper learning and understanding (Slavin, 1985; Vygotsky, 1978). Effective learning communities are based upon a collaborative learning theory whose fundamental idea is that learners must meaningfully collaborate in learning activities through interaction with others. In such an environment, learners interact with one or more peers to solve a given problem or achieve the same goal. A collaborative learning 
environment allows students to engage in a collaborative process of exchanging ideas and diverse viewpoints that increases their interest while promoting their critical thinking (Gokhale, 1995). The idea that people construct their own understanding by actively processing content in a collaborative learning environment to establish their own meaning is framed from a constructivist perspective that originates from Vygotsky's (1978) cognitive research.

One of the major ideas of Vygotsky (1986) is that human's higher cognitive functions develop through products of mediated activity. That is, cognitive processes are developed through social interaction, and individual consciousness is built from outside through relations with others. The process of intra-psychological interaction within oneself is indeed the representation of inter-psychological experience with others (Vygotsky, 1978). An important point in the social relation is the internalization by the learner of the processes of interaction. According to Gredler's (1997) view of this perspective, "relations between the higher mental functions were at one time real relations among people" (p. 254).

Vygotsky's work is based on the premise that the development of human cognitive functions differs under the influence of differing social and cultural experiences (Gredler, 1997). As one of the supporting grounds for the assumption, Vygotsky includes the critical function of language as a tool for learning. He notes that an individual's internalization of socially rooted and historically developed experiences relies on the mediation of language. Language is a communication medium not only for interacting with other people, but also for structuring and operating an individual's thinking. 
Hence, according to Vygotsky (1978), the internal operation of human learning and development is inherently social, as it is based upon the interactive experiences with other people by means of language that is essentially a social and cultural product itself. The social nature of cognition, as theorized by Vygotsky (1978), provides a basis for learning requiring social interaction, because Vygotsky viewed learning as a particularly social process with language and dialogue essential to cognitive development. Slavin (1990) developed Vygotsky's views of the functions of human interaction into educational practice. The linguistic exchange between and among students and the experience of collaborative learning in the construction of knowledge is emphasized. This requires students to share how they think and act as mediators of other students' thinking. Through dialogue, learners become more aware of their own thoughts as tools to apply tentatively to given problems, and in the dynamic exchange between and among their peers, teach themselves more powerful dimensions of thinking (Presseisen, 1992). This makes a distinction between the transmission of information and collaboration. Knowledge is perceived as a dialectic process in which individuals test their constructivist views on others and negotiate their ideas (Foley, 1995).

In a distance education context, a course that provides a means of regular communication through electronic communication and has a defined purpose for group interaction establishes a means of developing a socially supportive environment for students who are remote from one another. Such a group-supported environment is conducive to effective learning (Foley, 1995; Knowles, 1990).

In summary, a socio-cultural, constructivist cognitive approach to learning emphasizes that learning is an active and evolving process. Learners are engaged in 
integrating new information into existing knowledge structures. Through the ongoing interaction between instructor and student and student and student, development of meaningful and valid knowledge structures are encouraged (McIsaac, Blocher, Mahes, \& Vrasidas, 1999). According to McIsaac et al. (1999), and Gunawardena and Zittle (1997), studies have shown that computer-mediated conferencing has the potential for learners to engage learners in discourse and establish a learning community. However, Walther (1992) claims that several of the studies conducted on computer-mediated conferencing measure the effectiveness of the media by asking individuals to rate the media. Instead, the learner's satisfaction in using the media should be measured through examining the learner's perceptions.

Understanding the learners' experiences and perceptions of learning in computermediated communication provides the conditions under which learning takes place. Without the knowledge of the learner, instructors can only make assumptions about the learning process and use these assumptions to attempt strategies that may or may not improve learning in this environment.

\section{Importance of Perceptions}

Perceptions may be more important than they appear to be at face value, this is particularly the case in the adoption of a new product or way of doing things (Dormant, 1999; Rogers, 1995). "If men perceive a situation as real, that situation is real in all of its consequences, in other words perceptions count" (Rogers, 1995, p. 11). Winn (1993) has noted that perception is one of the earliest stages of cognition that must occur before deeper processing can. It is not enough for an idea or product to be effective or good, it 
must also be perceived as being such by the targeted audience for adoption to occur (Dormant, 1999; Rogers, 1995).

Several studies of student performance in face-to-face and online courses rely on student perceptions of their learning experiences including "how well" or "how much" they have learned (Picciano, 2002). Some of these report findings relative to learner and instructor perceptions of technology and learning.

In a review of the distance education literature, Zirkin and Sumler (1995) suggest that research demonstrates "the greater the interactivity in distance delivery, the more favorably the instruction was perceived" (p. 99). Jiang and Ting (2000) found a significant correlation between learners' perceptions of learning and their perceived interaction with the instructor. Westbrook (1999) found that, although students in their first online course anticipated the same level of interaction with the instructor in a typical face-to-face classroom, they felt they received more interaction with the instructor and with other students than was initially anticipated. Students also felt the online experience had allowed them more time to spend on course content.

Harasim and Yung (1993) and Harrison et al. (1995) found that learners felt that computer-mediated communication differed from classroom communication in that it offered greater access to teachers, increased communication among learners, offered more detailed discussion with greater depth of analysis, created greater independence among learners, and more time for reflection and exchange of ideas. In contrast, Cahoon (1998) found that some students do not appreciate learning in the online environment, expressing frustration at the isolation and absence of familiar social cues. Dede (1995), 
on the other hand, reported that shy or reflective people may find written exchanges in an online course to be more authentic than face-to-face verbal exchanges.

Individual perceptions of interaction have also been shown to be a critical predictor of learner satisfaction. Fulford and Zhang (1993), for instance, examined learner perceptions in a course delivered by instructional television. They found that the predictor of student satisfaction in the course was not the extent of the learners' personal interaction but the learners' perception of their own personal overall interaction. Researchers experienced with online teaching and learning contend that personal perceptions rather than media capabilities are important in promoting learning (Gunawardena \& Zittle, 1997; Rourke et al., 2001; Walther, 1994).

For example, Gunawardena and Zittle (1997) developed a survey to explore student perceptions of social presence in computer conferencing associated with a Global Education course that included participants in both the United States and Mexico. In two separate studies, they found that students rated asynchronous discussion as highly interactive and social. The researchers concluded that course participants created social presence by projecting their identities online and building a discourse community among themselves. What were important, they argued, were student perceptions of the presence of others, not the medium's capacity to capture gestures and intonations.

Conversely, frustration with the educational experience could lower performance and produce feelings of isolation or a poor attitude toward learning. For example, Hiltz (1998) noted a perceived loss of the sense of community when students compared online learning with traditional campus experiences. A study by Anderson (1997) sheds more light on this perception. This study found that $65 \%$ of the students, who were enrolled in 
their first online course, felt the online discussions were not as good as what would have been exchanged in face-to-face class meetings. In addition, $79 \%$ indicated they were limited in their ability to communicate and $82 \%$ felt it more difficult to socialize online and felt disconnected.

Reviewing studies such as these reflect that socio-emotional factors, such as the construct of social presence, can be related to instructional effectiveness, positively affecting learning and student satisfaction. Ultimately, student perceptions of their learning may be as good as other measures because these perceptions may be the catalyst for continuing to pursue learning opportunities through distance learning such as Webbased learning (Picciano, 2002). All things being equal, it is clear from the distance education literature that perceptions matter and there is value in examining them. Implications of Social Presence for Online Learning

The use of online instruction as an educational delivery method continues to expand across various settings. The environment in which learning takes place- whether online or in person- involves a complex array of factors that influence learner satisfaction and achievement. The physical space and psychological climate in which learning occurs, as well as social aspects of instructional activities, play an important role in successful learning (Hiemstra, 1991; Merriam \& Brockett, 1997; Sisco, 1991). The cognitive/social psychology and distance education literature identifies interaction among students as critical in learning and cognitive development (Gorham, 1988; Kelly \& Gorham, 1988; Sharan, 1980; Slavin, 1983; Vygotsky, 1978). There is a belief that high levels of interaction, particularly those which promote social engagement, can have positive effects on the learning experience. Vygotsky's emphasis on the social dimension 
in construction of knowledge has led many instructors to pay as much attention to how students learn as to what they are learning. With online learning, students can communicate in different temporal modes, either asynchronous or synchronous.

According to Bibeau (2001), teaching and learning functions are inherently social endeavors. The use of online learning has illuminated the effects of geographic, temporal, and psychological distance between students and instructors, therefore, it is beneficial to understand the various effects these have on teaching and learning between instructors and students. The lens through which these distances are examined is through the notion of social presence (Gunawardena \& Zittle, 1997).

The online learner needs a physical as well as a social context in an online discussion forum. Online discussions and interaction afford an opportunity to the student to bridge distance and time constraints in a social medium. However, Johnson and Huff (2000) noted that computer-mediated communication does not automatically impact learning. What then are some of the features of social presence that can impact learning?

Ultimately, social relationships contribute to group dynamics that are essential to developing a learning community (Rovai, 2002). The ability to participate in active collaborative learning is at the heart of social presence and of interest to those involved in creating communities of learners. Interactions among students seem clearly to matter in online discussion. Rourke et al. (2001) identifies the development of social presence as one of the cornerstones for the development of online learning communities.

A learners' sense of online community is integral to the concept of social presence (Fabro \& Garrison, 1998; Jonassen, 2000; Palloff \& Pratt, 1999; Wenger, 1998). Research shows that strong sense of community not only increases the persistence of 
students in online programs, but also enhances information flow, learning support, group commitment, collaboration, and learning satisfaction (Dede, 1996; Garrison, Anderson, \& Archer, 2003; Hornik \& Johnson, 2003; Wellman, 1999).

Social presence involves the ability of people to be perceived as real, despite not communicating face-to-face (Garrison et al., 2000; Gunawardena, 1995; Short et al., 1976; Tu \& McIsaac, 2002). The greater the perception that social presence exists, the better the ability to substitute telecommunications media for face-to-face encounters and still achieve the desired collaborative outcomes. When the degree of social presence is high, interaction most likely will be high. Interpersonal communication through reflection, exchange, and transaction is fundamental to individual and social presence in learning (Wulf et al., 2000). This serves as a way to construct knowledge and negotiate meaning through active learning (Palloff \& Pratt, 1999).

There are valuable aspects of social presence that include communication (dialogue and conversation), collaboration, interaction, and interpersonal skills. Wegerif (1998) asserts the importance of social factors in learning, course design, the role of instructors as facilitators, the interaction styles of course participants, and features of the technological medium used. Studies conducted on social presence, for the most part, have been in traditional classrooms. Results from these studies indicated that social presence is a significant factor in instructional effectiveness, positively affecting learning, student satisfaction, achievement, and motivation (Blocher, 1997; Christophel, 1990; Gunawardena \& Zittle, 1997; Hackman \& Walker, 1990; Rourke, 1999). However, very few studies have examined social presence in computer-mediated communications. 
The limited number of studies that do examine social presence in the context of computer-mediated communication do not cover the scope of how distance learning students perceive that social presence contributes to their learning satisfaction, learning experience, motivation to participate, or likelihood to enroll in future online courses. Since learner-centered instruction is a desired teaching strategy, a better understanding of students' perceptions of the extent that social presence contributes to their learning experience online may provide insights of student engagement in the learning process. Likewise, this may address the effects mediated communication has on group dynamics and the establishment of a sense of learning community.

However, there is no widely accepted measure of social presence. Short et al.(1976) used semantics differential scales to assess the social and emotional capabilities of the medium. Gunawardena and Zittle (1997) developed a scale that consisted of 14 Likert items addressing the social presence of a computer-mediated conferencing environment, particularly the concept of immediacy. They found that social presence is a strong predictor of overall course satisfaction. Garrison et al. (2000) developed a template for analyzing and coding transcripts from a computer conference in terms of cognitive, social, and teaching presence. This template provides categories that indicate elements of social presence. Richardson and Swan (2003) and Swan et al. (1998) used a survey to explore perceptions of social presence in online courses and found that perceptions of social presence and satisfaction with instructors were highly correlated. $\mathrm{Tu}$ (2002) developed a scale that consisted of 17 Likert items addressing social presence and 13 privacy items and asserts that social presence is a complicated construct and 
involves privacy, social relationships, communication styles, and the nature of the task, feedback, and immediacy among other items

What the above studies indicate is that distance education is a complex, dynamic system of teaching and learning. As in many complex environments, each element of the conceptual frameworks listed above illuminates a different facet of the way in which learners interacted with the instructor and one another during the course. Given the different measures of social presence, it might be best to combine some of these validated instruments to extend our understanding of social presence in online learning.

The literature in the field of communication and social psychology recommends considering the interrelationship between the communication concepts of intimacy, immediacy, and interactivity (interaction). Together these concepts form the construct of social presence. Given the lack of social presence research in distance education, McIsaac and Gunawardena (1996) voiced the need for additional studies on the dimensions of social presence comprised of intimacy, immediacy, and interactivity.

The factors determining interaction, intimacy, and immediacy that represent the construct of social presence are as twisted and interwoven as a thick braid. The model of delivery and quality of instruction represent only one axiom along which online dynamics can be understood. Another less controllable, less predictable, and perhaps more dynamic set of factors resides in the lives and hearts of the learners themselves. These factors are brought to the learning experience in the form of social presence that is manifested in levels of community, collaboration, and participation. 


\section{Summary}

The research literature indicates there are several forces that shape learning; in particular, these include the instructor, the student, and the tools used in the process (Thompson, 1995; Wenger, 1998). Technology is not a primary determinant of learning. Research has indicated, "technology is not nearly as important as other factors, such as learning tasks, learner characteristics, student motivation, interaction, and the instructor" (Institute for Higher Education Policy, 1999, p.8). Regardless of the method of delivery, the most important factors for successful distance learning involve interaction, between instructor and students, between students and the learning environment, and among students themselves. For students to learn, courses must be engaging, provoking them to communicate, share ideas, and think critically (Bennett \& Green, 2001).

Presently, the limited studies on social presence in distance education, particularly online learning, do not lend themselves to fully understanding the effects it has on the learning process. Current research, then, seems to indicate a heightened need for examining the relationship between social presence and perceived learning and satisfaction as well as retention in online courses. While social presence was initially concerned with qualities of the medium (Short et al., 1976), it has been demonstrated by research studies that this may not in fact be the case. Instead, other researchers (Gunawardena \& Zittle, 1997) claim that social presence should be considered a quality of the perception of users of a medium. Incorporating social presence into this new communication delivery medium provides an avenue to a sound learning environment. Understanding how the perception of social presence may influence student satisfaction and quality of learning in course activities may provide course designers with important 
information on how to positively influence retention in online courses. Further research on learning in an online environment is necessary to guide educators in planning, designing, managing, and delivering the best educational environment for learner achievement and satisfaction (Wynia, 2000). 


\section{CHAPTER III}

\section{METHODOLOGY}

The methodology is organized according to the following topics: (a) statement of

purpose; (b) research design; (c) selection of participants; (d) Internet survey research; (e) instrumentation; (f) data collection; (g) data analysis; and (h) limitations.

Statement of Purpose

Research into the principle of social presence in online courses is a relatively new research area. Although social presence has been characterized as an important construct in distance learning because it may exert significant influence on improving instructional effectiveness (Gunawardena \& Zittle, 1997; McIsaac \& Gunawardena, 1996; Tu \& Corry, 2001), little existing research describes its value to learners and whether it affects their perception of learning and retention within a computer-mediated environment. The purpose of this study is to examine social presence and its influence on satisfaction with learning and retention in an online learning course. This study is guided by the following research questions:

1. What is the relationship between learners' perceptions of social presence and their perceived satisfaction with learning in online courses?

2. What is the relationship between learners' perceptions of social presence, satisfaction with learning experiences and quality of learning in online course 
activities, e.g., class discussions, group projects?

3. What is the relationship between the perceptions of social presence, satisfaction with learning, and the likelihood of future enrollment in online courses?

\section{Research Design}

In this study, a basic correlational design was utilized (Gall, Borg, \& Gall, 1996). The principal advantage of the correlational method is that it permits the analysis of relationships among a large number of variables in a single study. The correlational method also provides information concerning the strength and the degree of the relationship between the variables being studied (Pagano, 1998). A hierarchical regression analysis was employed to determine the unique variance and effect explained by social presence and learning in predicting future enrollment and persistence in online courses (Cohen, 1988). An a priori determined conceptual model suggesting a mediating influence of social presence on learning and intent to enroll in future online courses was tested.

\section{Selection of Participants}

The sample for this study was selected from students enrolled in Web-based online courses at the University of Louisville during one semester. Recent studies on social presence (Gunawardena \& Zittle, 1997; Tu, 2002) have indicated a need for an investigation of a diverse sample of content, e.g., business, education, psychology, and sociology. The authors further recommended that students be selected with individual differences on such variables as level of college credits, overall computer experience, and online learning experience. The Delphi Center for Teaching and Learning of online 
courses at the university served as a resource in their recommendations and provided contacts for those courses that are delivered totally online for this study.

Based on a fall semester 2005 enrollment report from the Delphi Center for Teaching and Learning, the number of online courses at the University of Louisville was 115 (including undergraduate and graduate level) with an approximate total of 1900 registered students. For a course to be considered as online distance education, $75 \%$ of the course must be online. Course enrollment for distance learning during the fall semester ranged from a minimum of 5 to a maximum of 59; course enrollment averaged approximately 25 students.

The literature review on Internet surveys indicates that electronic survey response rates are much lower than either traditional mail or telephone surveys (Schonlau, Fricker \& Elliott, 2001). A recent Web survey conducted with students in two-part studies at the University of Michigan yielded an average response rate of 30\% (Crawford, Couper \& Lamias, 2000; 2001). For the purposes of this study, permission was requested from all online course instructors identified by the Delphi Center for Teaching and Learning in order to reasonably obtain at least a $30 \%$ response rate from course instructors willing to participate in the study. It was estimated that $12-15$ course instructors might permit access to their enrolled group of students (averaging approximately 25 per course) to provide data collection from an estimated 112 volunteer participants for this study.

All courses provided online at the University of Louisville employ Blackboard 6. 0 course-management system as a Web-based application that facilitates the provision of course information, the distribution of learning resources, communication between instructors and students and among students, file sharing, and online feedback and 
assessment. The Delphi Center for Teaching and Learning provides instructors with training in the use of the course management system and in instructional design. The Center also provides access to ongoing support. Surveys that are delivered online into the course Blackboard are created via the Delphi Center through a protected password using a survey tool known as Zoomerang.

To minimize the number of factors that could influence validity, certain criteria were employed in the selection of course participants. Courses that were web-assisted or web-enhanced were not included because the main focus of this study was to elicit perceptions of students' online experiences in terms of social presence. This means physical contact was not available between the instructor and students. In addition, firsttime online courses were excluded from this research because the complications that can result from a new delivery mode for a course could possibly bias students' perceptions of social presence. A similar sample of online courses that excluded first-time online delivery was selected in a recent study (Richardson, 2001) due to the complications and complaints that generally plague newer online programs. Permission was requested from the respective course instructors to access their enrolled group of students.

Based upon the prerequisite criteria used for the purpose of this study, 32 of 115 courses $(28 \%)$ did not meet the requirements because these courses allowed for campus participation in addition to online instruction (web-enhanced) or they were offered for the first time. Eleven (10\%) course instructors indicated they did not wish to participate in the study. Thirty (26\%) course instructors did not respond. Forty-two (37\%) course instructors granted permission to participate in the study, however, three of those did not meet the required enrollment and were cancelled resulting in a net gain of $39(34 \%)$ 
courses that granted permission to access their students for the purposes of the study. This surpassed the $30 \%$ response rate that was initially anticipated. In summary, out of the 83 identified potential distance education courses that were offered fall semester and met the prerequisite criteria, $39(49 \%)$ course instructors granted permission to access the enrolled students for the study. These courses included both undergraduate (21) and graduate level (18) and involved various disciplines such as communication (8), computer engineering and computer science (2), education (6), human resource development (8), justice administration (6), Pan-African studies (3), psychology (2), sociology (3), and nursing (1).

Before conducting the research activities, approval was obtained from the Human Subjects Committee at the University of Louisville explaining the intent, the process, and the value of an individual's participation in the research that would possibly influence future delivery of online courses.

All potential course participants were notified about the research and the forthcoming survey through an email message launched into the respective course Web site using the communication link set up by the instructor (Appendix A). This occurred by the sixth week of the semester to allow sufficient time for first-time online students to be knowledgeable regarding use of the Blackboard 6.0 system and have time to experience various course aspects of learning online. This methodology is supported by Hillman (1999) who recommends that an online instructional program should take into consideration the time and other factors that could allow first-time online learners an opportunity to interact productively and frequently and possibly result in formation of a learning community. 
Upon receiving the solicitation email and selecting the URL website, the student participant was presented with a confidentiality statement informing participants as to how the data would be used and reported as well as instructions to assist participants in completing the survey instrument. Participants' email addresses were accessed individually through the communication link on the course site so as not to disclose any individual's email address. Participation was voluntary and students were able to opt out of the study at this point.

Students who elected to participate were directed to a specific URL site for their course (Appendix B). Each course was provided a unique course code. The purpose of this code was to allow the response rate from each class to be tracked. The students' data was recorded in a file after submitting the completed survey. Because the file did not contain any identifying information, participant anonymity was assured. Participants' responses were kept confidential. In addition, responses were not shared with the faculty member, which might have interjected bias into the study (Gall, et al., 1996).

\section{Internet Survey Research}

One important technique used for gathering data in education and business is through the survey. Surveys are "a means of gathering information that describes the nature and extent of a specified set of data ranging from physical counts and frequencies to attitudes and opinions" (Isaac \& Michael, 1995, p. 128). Surveys are also a key research instrument for gathering both quantitative and qualitative data from a selected group of individuals (Creswell, 1994; Fink, 1995; Fowler, 1984).

The use of the Internet has continued to increase at a phenomenal rate. The percentage of higher education institutions offering distance education courses has 
increased by one-third during the past decade as well (National Center for Educational Statistics, 1999). Moreover, the changes in the hardware and software have improved the access to the Internet. These recent developments have resulted in a growing interest among both academic and organizational researchers regarding the feasibility of using the Internet as a means of data collection, particularly with the use of Web-based surveys (Kiesler \& Sproull, 1986; Schmidt, 1997; Stanton, 1998).

Self-report surveys are arguably the most widely used form in organizational and institutional studies and are relatively easy to facilitate through modalities such as electronic mail and the World Wide Web (Kraut, 1996). Internet-based surveys have become very prevalent and may replace telephone surveys and personal interviews in certain situations because of a number of advantages (Manfreda, Batagelj, \& Vehovar, 2002).

\section{Advantages/Strengths of Internet Survey}

Advantages of Internet-based surveys cited in the literature include: design flexibility and interactivity, ability to reach large numbers of people, anonymity, economy, greater time efficiency than mail and telephone surveys, minimized interviewer error, and minimized interviewer bias (Sheehan \& Hoy 1999; Schneider \& Dillman, 1998; Simsek \& Veiga, 2001).

Both email and the World Wide Web are primarily used for Internet self-report surveys. The email survey involves a computerized, self-administered questionnaire sent out by the researcher, which the respondents receive, complete, and return through email (Simsek \& Veiga, 2001). Such surveys can include the following methods: (a) sending an email message with the survey as part of the message text; (b) sending the survey as 
an attachment to an email message that the respondent must open in order to respond; and (c) sending an email message with a URL-embedded message in the text that the respondent is directed to click on a hypertext link that allows the recipient to view and respond to a Web-based survey (Simsek \& Veiga, 2001).

\section{Disadvantages/Weaknesses of Internet Survey}

No single survey approach can be judged as the most effective means to gathering data because each approach is situational (Kiesler \& Sproull, 1986; Lockhart \& Russo, 1996). There are potential problematic and controversial aspects of Internet surveys including: technical problems (Yun \& Trumbo, 2000); sampling error, coverage error, and generalizability issues with populations that have Internet access or usage problems (Crawford et al., 2001; Dillman, Tortora, \& Bowker, 1999; Sheehan \& Hoy, 1996); response rates typically lower than traditional mail surveys or nonresponse error (Crawford et al., 2001; Crawford, Traugott, \& Lamias, 2001; Dillman \& Bowker, 2001); measurement error, and possibly security issues (Sills \& Song, 2002). All of these types of errors must be kept at a minimum for any sample survey to claim that it is a relatively precise estimate of the distribution of a characteristic within a population.

There are some mitigating factors to be considered with regard to the potential disadvantages. While it is true that serious technical difficulties have been reported (Crawford et al., 2001), little research has been done that compares the frequency of technical or logistical problems between the electronic and traditional survey modes.

Dillman et al. (1999) defined coverage as "all units of a population not having a known probability greater than zero of inclusion in the sample that is drawn to represent the entire population" (p.2). Coverage error, therefore, results in some units within the 
population having no chance of selection; others having multiple chances; and still others not even qualifying for the survey. Coverage error is reduced when each member of a defined population is given a chance of being surveyed and there is a high response rate (Dillman \& Bowker, 2001).

Sampling error was defined by Dillman et al. (1999) as "surveying a portion of the population rather than all of its members"(p. 3) whereas measurement error is defined as "the result of inaccurate answers to questions that stem from poor question wording, poor interviewing, survey mode effects, and/or answering behavior of the respondent" (p.2).

Sampling non-coverage and concomitant generalizability problems are very important issues that must be contended within an Internet self-administered survey, but these may be lessening as computer and Internet access and usage are becoming more prevalent among certain population groups (Crawford et al., 2001; Forsman \& Varedian, 2002; Lesser \& Newton, 2002; Sills \& Song, 2002). For example, coverage problems are minimized with university faculty and students as such groups are likely to have reliable email address lists (Crawford et al., 2001). This affords the opportunity for a sampling approach to survey as many identifiable targets as possible (Bradley, 1989); particularly if only one organization is involved (Simsek \& Veiga, 2001).

Shannon and Bradshaw (2002) reported that, although some researchers using electronic surveys have achieved response rates as good or better than traditional mail and telephone surveys, most have found electronic survey response rates to be lower. Response rates for Internet surveys have ranged from as low as $7 \%$ to as high as $76 \%$ (Sheehan \& Hoy, 1999; Simsek \& Veiga, 2001). According to a recent literature review 
on Internet surveys, Schonlau et al. (2001) found that Web survey response rates ranged from $6 \%$ to $68 \%$. This study further found that when a mail and a Web response mode were used together the response rate increased compared to those surveys that were conducted using only an email or Web response mode.

In two-part studies done by Crawford et al. (2000; 2001) using Web surveys with students at the University of Michigan, response rates ranged from 23\%-37\%. Crawford et al. (2002) recently achieved a $63 \%$ response rate with the same population. However, the authors were able to offer an incentive of a $\$ 10$ gift certificate for every participant redeemable at the local bookstore. Such incentives are usually beyond the means of most research studies and may also be a source of potential bias.

By contrast, response rates for mailed surveys tend to be higher. Babbie (1990) contended that a return rate of $50 \%$ is adequate. Mangione (1998) suggested that response rates of $60 \%-85 \%$ are considered acceptable and viewed as very good while response rates below $60 \%$ are barely acceptable and viewed as poor as well as reducing confidence about the quality of the data. Dillman (2000) provided methodological guidance for conducting mail surveys and suggested that mail survey response rates of $50 \%-70 \%$ are common.

For both mail and email surveys, pre-notification has been seen to increase response rate (Murphy, Daley, \& Dalenberg, 1991; Sheehan \& McMillan, 1999; Taylor \& Lynn, 1998). Mehta and Sivadas (1995) suggested that pre-notification for email surveys is imperative, and the practice of sending unsolicited email surveys is unacceptable. 
One of the most important techniques for producing high response rates is the use of reminders. With both the use of a mail or an Internet survey, additional time can be realized when there is more than one contact with respondents, such as mailing reminders or sending follow-up questionnaires (Dillman, 1978; Simsek \& Veiga, 2001). Dillman (1978) prescribed that reminders be sent one, three, and seven weeks after the mailing of a survey. Shannon and Bradshaw (2002) reported that it took an average of 9.13 days to receive a survey response by mail compared to 3.21 days for electronic surveys. Crawford et al. (2001) tested the effect of shortening the waiting period of a reminder to only two days between the initial invitation and the first reminder. They found this approach had only a modest positive impact on the speed of the returns and the overall response rate.

Although there are trade-offs when deciding to use Internet surveys to collect data, it is important to give appropriate attention to their limitations from a comprehensive perspective and follow suggested strategies that can be used to overcome some of the limitations (Simsek \& Veiga, 2001). The value of any new surveying technique, such as the Internet survey, will depend on how to handle the issues faced by researchers with most instruments and still take advantage of the unique strength of this new method.

In summary, possible limitations were taken into consideration in using an Internet survey for this study by following suggested strategies in published studies that address sampling, measurement error, generalizability, security, and response rate issues. For example, coverage and sampling problems were minimized by targeting university students in one organization that have been identified as a representative sample of 
students who are enrolled in a Web-based online learning experience. These individuals would have reliable email address lists that afforded the opportunity to survey as many identifiable targets as possible. Measurement error was minimized by using results from the feedback of a pilot-study that served as a formative evaluation tool to test the overall methodology, item construction, and clarity of wording that resulted in refinement of the survey instrument. The issue of security was addressed by using the communication link on Blackboard for each course Web site and inserting individual participants' email addresses into the email notification so as not to disclose any individual's email address. Participants were also informed of the confidentiality as to how the data would be used and reported.

For each completed survey, the data were recorded in a file stored on the server through the Delphi Center that also housed the survey. Because the file did not contain any identifying information, participant anonymity was assured. To maximize response rate, pre-notification and multiple follow-up contacts were sent during the data collection period of the study. Pre-notification and contact frequency has been shown to result in improved response rates (Mehta \& Sivadas, 1995; Murphy et al., 1991; Schaefer \& Dillman, 1998; Sheehan \& McMillan, 1999).

\section{Instrumentation}

The survey instrument used in this study is primarily based on a social presence scale originally constructed by Gunawardena and Zittle (1997) for their research examining social presence as a predictor of satisfaction within computer-mediated conferencing environments (Appendix C). The primary purpose for selecting this 
instrument was because the construct of social presence, the perception of student learning, and the variable of student satisfaction were measured.

The social presence scale developed by Gunawardena and Zittle (1997) consisted of 14 questionnaire items that measured immediacy; a 10-item scale that measured both overall satisfaction with the conferencing medium as well as student learning perspectives. Five-point Likert-scale items were constructed to assess self-report measures of satisfaction and social presence along with other variables. Data were gathered from 50 graduate students. Multi-item scales were analyzed for internal consistency using Cronbach's alpha. Reliability analysis of the instrument ranged from alpha of .72 to .88 , with the social presence scale resulting in the highest alpha (.88). The satisfaction and learning perspectives scale resulted in an alpha of .87. Stepwise regression analysis showed that social presence accounted for $60 \%$ of the variance in the dependent variable, suggesting that it had potential as an indicator of satisfaction.

The survey instrument for this study was also based on a scale originally constructed by $\mathrm{Tu}(2002)$ for his research examining social presence and interaction as a predictor of satisfaction within the computer conferencing systems of email, bulletin board, and real-time discussion. The primary purpose for selecting this instrument was its capacity to measure both social presence and interaction. Interaction has been shown to have a strong relationship with social presence and the development of a learning community. The effects of interaction are a common theme in distance education research.

The Social Presence and Privacy Questionnaire developed by Tu (2002) evaluating email, bulletin board, and real-time discussion contained 17 social presence 
items along with 13 privacy items each with a 5-point Likert scale. Data were gathered from 43 education graduate students at a major university. Confirmatory factor analysis was used to examine the indicators of social presence, particularly online interaction/communication, and the privacy factor. High alpha values were reported for all factors and significant correlations were found between all factors. The study revealed that social presence in an online environment is the degree of feeling, perception, and reaction to another intellectual entity in the computer-mediated communication (CMC) environment. The author also concluded that when a learner perceives a higher degree of social presence, there is a greater likelihood that a higher level of interaction among learners will occur.

The author modified both survey instruments from the original in several ways. First, the language was modified to correspond with the University of Louisville environment rather than the specific environments in either instrument they were originally intended to study. The instrument designed by Gunawardena and Zittle (1997) was constructed to assess self-report measures of social presence and satisfaction through a GlobalEd inter-university computer conference. An example statement of this social presence scale is, "I felt comfortable in participating in GlobalEd discussions". This has been modified to reflect the language within an online environment. An example statement to illustrate this is, "I felt comfortable in participating in course discussions". The two scales were combined so that the two dimensions of social presence (interaction and immediacy) could be measured. The focus was not only on the perception of social presence and satisfaction but also on students' perceived learning. This subscale corresponds to items 1-23 in Section Two of the instrument. 
The scale was also modified so that individual course activities, e.g., discussion, group projects, written assignments, could be examined in view of the fact that the original scales examined a course from an overall perspective (Gunawardena \& Zittle, $1997)$ or the tools of communication used $(\mathrm{Tu}, 2002)$ in a specific course. This subscale corresponds to items 5-10 in Section Three of the instrument. Each of these course activities have been successfully investigated in a previous research study $(n=97)$ of social presence and instruction in an online course environment (Richardson, 2001). The results revealed social presence (e.g., immediacy) contributed significantly to the students' perceived learning for individual course activities within an online course. This study endeavored to extend this research and examine social presence and its contribution to perceived quality of learning in predicting future enrollment in an online course. Pilot-Study

According to Gall et al. (1996), evidence of content validity is obtained by acquiring agreement from content experts. Content validation of the survey was executed through an examination of the instrument by three distance learning experts. Any perceived limitations, following their review, were addressed through suggested changes to the survey.

Following this procedure, the survey was administered to a small group of graduate students (24) who had completed at least one online class and who were enrolled in a Web-based asynchronous online course during the summer term. Participation was voluntary. These students were not selected as participants in the final study for the purposes of reliability and content validity. The pilot test served as a formative evaluation tool to test the effectiveness of the instrument so that the resulting 
measurements would be accurate and reliable (Gall et al., 1996). The participants were asked to examine the wording of the survey for clarity of instructions and questions. In addition, they were asked to assess survey items for their completeness in covering the activities involved in online courses and asked to recommend any additional items or activities to be included in the final survey. Moreover, this formative evaluation served to test the overall methodology of the research design.

Nine participants $(37.5 \%)$ responded to the survey. The results from the feedback of the pilot-study resulted in further refinement of the instrument by making only a few grammatical changes within the instructions to make the survey easily understood. No additional items or activities involved in online courses were recommended. The pilotstudy also confirmed that the online version of the instrument was easy to access, complete, and submit, and that the questionnaire was secure and reliable.

The pilot survey instrument consisted of four sections. Section One of the instrument consisted of demographic items (age and gender) and relevant background student characteristics as identified from the literature. Background characteristics consisted of amount of online experience (number of courses), number of college credits, and reason for participating in an online course. The purpose for these questions was to provide a description of the participants. Two of the four sections employed a Likert-type scale designed as self-report measures to explore the variables of interest in this study. The final section of this research instrument consisted of open-ended questions that were used to gather qualitative data that could lead to convergent evidence concerning the importance of social presence. 
Likert-scale items are useful for gathering respondents' opinions, feelings and attitudes, and the extent to which a person agrees or disagrees with questions (Brown, 2000). A Likert-scale was chosen for this study because it allows respondents to express both the direction and strength of their opinion about a topic. A further advantage of the Likert-scale is that it has been extensively tested in the social science literature and data can be treated quantitatively (Garland, 1991). The most common scale used is 1 to 5 . The items for this instrument used a six-point response scale $(1=$ strongly disagree to $6=$ strongly agree). As there is a tendency for people to respond towards the middle of the scale, perhaps to make them appear less extreme, an even number of options was used to compel those respondents who generally choose a neutral option to choose either in the positive or negative direction so that a definite opinion may be expressed (Brown, 2000; Mattell \& Jacoby, 1972). Students were prompted to indicate the degree to which they agreed with each statement and provide a greater opportunity to capture the variance.

Section Two of the instrument consisted of 23 Likert-type items selected from the combined and modified social presence scales (Gunawardena \& Zittle, 1997; Tu, 2002) to assess students' overall perceived social presence and students' overall perceived satisfaction with learning in their respective online course. In addition, respondents were asked to assess and self-report on a six-point scale their satisfaction with learning using a modified Satisfaction with Learning Scale. This subscale was derived from the Workplace Adaptation Questionnaire (Morton, 1993). This 22- item instrument consisted of three subscales measuring employee socialization-related learning and one subscale measuring satisfaction with learning experiences. The satisfaction with learning experiences subscale evaluated employees' satisfaction with their learning experiences at 
the organization. An example statement of this subscale is, "I am satisfied with the feedback I have received about my performance on the job". This subscale (items 24-28) has been modified to reflect the language within an online environment rather than within an organization. The internal reliability for this scale has been reported as an alpha of .83 (see Reio [1997] for a detailed examination of this instrument).

Section Two was also composed of 12 Likert-type items prompting the student to indicate the degree to which they agree (1= strongly disagree to $6=$ strongly agree $)$ regarding the value or importance the students place on the construct of social presence within the context of the Web-based course. This subscale corresponds to items 29-40. The basis for the design of these items will include the components of intimacy, immediacy, and interactivity (as determined by the literature) to form the survey questions for this section (Argyle \& Dean, 1965; Gorham, 1988; Heath \& Bryant, 1992; Mehrabian, 1969; Moore, 1989; Short et al., 1976; Wagner, 1994, 1997; Wiener \& Mehrabian, 1968). In addition, this section included four Likert-type items (41-44) that prompted the student to indicate the likelihood of the student's intent to enroll or not in a future online course. This scale was modified from the Intention to Turnover Scale that has been investigated successfully in previous studies related to organizational commitment (Abraham, 1999). The questions taken from the Intention to Turnover Scale, part of the Michigan Organizational Assessment Questionnaire (Cammann, Fichman, Jenkins, \& Klesh, 1979), was modified to gather respondents' opinions regarding the likelihood that they will enroll in future online courses. The internal reliability of this scale has been reported as an alpha of .83 . 
A few of the items in Section Two of the instrument were phrased in such a way that reverse scoring will be required prior to forming the scale mean. An example is item 16, It is difficult to express what I want to communicate online. The scoring rule used for each item in Section Two of the instrument is shown in Table 1.

\section{Table 1}

Scoring Rule for Section II of Instrument

\begin{tabular}{|l|l|}
\hline $\begin{array}{l}\text { Item Number of Section } \\
\text { II }\end{array}$ & $\begin{array}{l}\text { Scoring Rule: } \\
\text { + means positives scoring } \\
\text { - means negative scoring }\end{array}$ \\
\hline 1 & + \\
\hline 2 & + \\
\hline 3 & + \\
\hline 4 & + \\
\hline 5 & + \\
\hline 6 & + \\
\hline 7 & + \\
\hline 8 & + \\
\hline 9 & + \\
\hline 10 & + \\
\hline 11 & + \\
\hline 12 & + \\
\hline 13 & + \\
\hline 14 & + \\
\hline 15 & + \\
\hline 16 & + \\
\hline 17 & + \\
\hline 18 & + \\
\hline
\end{tabular}




\begin{tabular}{|c|c|}
\hline 20 & - \\
\hline 21 & + \\
\hline 22 & - \\
\hline 23 & + \\
\hline 24 & + \\
\hline 25 & + \\
\hline 26 & + \\
\hline 27 & + \\
\hline 28 & + \\
\hline 29 & + \\
\hline 30 & + \\
\hline 31 & + \\
\hline 32 & + \\
\hline 33 & + \\
\hline 34 & + \\
\hline 35 & + \\
\hline 36 & + \\
\hline 37 & + \\
\hline 38 & + \\
\hline 39 & + \\
\hline 40 & + \\
\hline 41 & - \\
\hline 42 & + \\
\hline 43 & + \\
\hline 44 & - \\
\hline
\end{tabular}


Overall, Section Two was comprised of a 44- item, self-report questionnaire based on the respondents' perceptions of the measures of interest in this study (dimensions of social presence, satisfaction with learning, and likelihood of future enrollment in an online course). From an examination of the literature, the subscales selected for use in Section Two most closely meet the needs of this study and have demonstrated psychometric rigor.

Section Three of the instrument consisted of indicator statements selected by the researcher from the modified combined scales (Gunawardena \& Zittle, 1997; Tu, 2002) that related to social presence, interaction and satisfaction, as well as indicator statements that measured perception of improved quality of learning through online course activities. These indicator statements queried the student about several types of course activities selected based upon their natural occurrence in the courses included in the study. These included such activities as the following:

1. Class discussion (question and answer)

2. Individual projects

3. Group projects

4. Reading assignments

5. Writing assignments

For each of these course activities, students were prompted to indicate the degree to which they agree with each of the indicator statements using a six-point Likert-type scale $(1=$ strongly disagree to $6=$ strongly agree $)$. Students were allowed to answer "Not Applicable" if the course activity was not present in their online course. For each of these 
course activities, a perceived social presence and a perceived quality of learning variable were obtained.

Items 1-4 in Section Three correspond to perceived quality of learning based on a questionnaire used in a three-year longitudinal field study of 26 online courses at a major university (Hiltz et al., 2000). These items were created to measure the quality of learning based on the variables of social presence, active involvement and collaboration. The reported internal reliability as measured by Cronbach's alpha for these items was .93 . An example of an indicator statement related to quality of learning is "I gained more interest in the subject". The empirical evidence of this field study indicated that the combination of collaboration and social presence enhanced student perceptions of the quality of learning.

Items 5-10 in Section Three are indicator statements based on the modified social presence scales from Gunawardena and Zittle (1997) and $\mathrm{Tu}$ (2002). The purpose of this examination in the current study was to investigate the relationships between perceptions of social presence in individual course activities and quality of learning for the students. An example of an indicator statement is "Online or web-based education is an excellent medium for social interaction as demonstrated by this activity."

A mean score for perceived social presence and perception of quality of learning based on a six-point Likert scale was computed individually for the five course activities listed above. A high score indicated a learner perceived a higher degree of social presence and quality of learning for a particular course activity.

Through the use of this study's research instrument, qualitative data were also gathered to determine students' perceptions of the benefits associated with course 
activities in terms of their quality of learning and satisfaction. In Section Four of this instrument, qualitative data were gathered to determine the value of these experiences within the course to predict future enrollment in such courses. Using both quantitative and qualitative research methodologies can lead to convergent evidence concerning the importance of social presence (Gall et al., 1996).

The qualitative section of this instrument was comprised of open-ended questions that students answered pertaining to their perceptions of benefits, in terms of their quality of learning and satisfaction, related to the five course activities. An example of such an open-ended question is: "Which of the course activities listed did you find most beneficial to your learning and why?" Another open-ended question was related to the students' intent to enroll or not in a future online course based upon their perceived quality of learning and satisfaction with their online course experience. An example question is: "As a result of your experience within this course, would you consider taking another Web-based online course? Briefly explain."

Table 2 provides a derivation of the various instruments that were selected for each section of the survey and the variables they examined in this study (See Appendix C for a copy of the final questionnaire). 


\section{Table 2}

Derivation of Instruments for the Study

\begin{tabular}{|c|c|c|c|c|}
\hline $\begin{array}{l}\text { Section of } \\
\text { instrument }\end{array}$ & Number of items & $\begin{array}{l}\text { Variable(s) } \\
\text { measured }\end{array}$ & $\begin{array}{c}\text { Source of items } \\
\text { adapted from } \\
\text { previous } \\
\text { instrument } \\
\end{array}$ & $\begin{array}{c}\text { Published } \\
\text { reliability } \\
\text { (Cronbach's } \\
\text { Alphas) } \\
\end{array}$ \\
\hline $\mathrm{I}$ & 13 & $\begin{array}{l}\text { Demographic } \\
\text { variables, Online } \\
\text { experience, Number } \\
\text { of college credits, } \\
\text { Reasons for taking } \\
\text { the course }\end{array}$ & & \\
\hline $\begin{array}{l}\text { II } \\
\text { Part 1. Items } 1-23\end{array}$ & 23 & Social presence & $\begin{array}{l}\text { Gunawardena \& } \\
\text { Zittle (1997); } \\
\text { Tu (2002) }\end{array}$ & $.88 ; .87$ \\
\hline Part 2. Items 24-28 & 5 & $\begin{array}{l}\text { Satisfaction with } \\
\text { learning experiences } \\
\text { subscale }\end{array}$ & Morton (1993) & .83 \\
\hline Part 3. Items $29-40$ & 12 & $\begin{array}{l}\text { Components of } \\
\text { Social presence } \\
\text { (intimacy, } \\
\text { immediacy, and } \\
\text { interactivity) }\end{array}$ & $\begin{array}{l}\text { Argyle \& Dean, } \\
\text { 1965; Gorham, 1988; } \\
\text { Heath \& Bryant, } \\
\text { 1992; Mehrabian, } \\
\text { 1969; Moore, 1989; } \\
\text { Wagner, 1994. 1997; } \\
\text { Wiener \& } \\
\text { Mehrabian, } 1968\end{array}$ & \\
\hline Part 4. Items 41-44 & 4 & $\begin{array}{l}\text { Intent to enroll in a } \\
\text { future online course }\end{array}$ & Abraham (1999) & .83 \\
\hline III & & & & \\
\hline Part 1. Items 1-4 & 4 & Quality of learning & $\begin{array}{l}\text { Hiltz, Coppola, } \\
\text { Rotter, Turoff, \& } \\
\text { Benbunan-Fich } \\
\text { (2000) }\end{array}$ & .93 \\
\hline Part 2. Items 5-10 & 6 & $\begin{array}{l}\text { Social presence in } \\
\text { course activities }\end{array}$ & $\begin{array}{l}\text { Gunawardena \& } \\
\text { Zittle (1997); } \\
\text { Tu (2002) }\end{array}$ & $.88 ; .87$ \\
\hline $\begin{array}{l}\text { IV } \\
\text { Qualitative Items 1-4 }\end{array}$ & 4 & $\begin{array}{l}\text { Quality of and } \\
\text { Satisfaction with } \\
\text { learning in course } \\
\text { activities }\end{array}$ & & \\
\hline Item 5 & 1 & $\begin{array}{l}\text { Intent to enroll in a } \\
\text { future online course }\end{array}$ & & \\
\hline
\end{tabular}




\section{Data Collection}

As indicated previously, all potential course participants were notified about the research and the forthcoming survey by the sixth week (Appendix A). This was accomplished through an email message using the Blackboard communication tool to inform the selected subjects that they would receive the survey by the seventh week of the semester (Appendix A). Pre-notification has been shown to increase response rate (Sheehan \& McMillan, 1999). This allowed sufficient time for first-time online students to be knowledgeable regarding use of Blackboard 6.0 system and have time to experience various course aspects of learning online.

The message contained a confidentiality statement informing participants as to how the data would be used and reported as well as instructions to assist participants in completing the survey instrument. Voluntary participants were assured that their responses would be kept confidential. In addition, they were assured that their responses would not be shared with faculty, which might have interjected bias into the study (Gall et al., 1996).

The email message included a "quick link" to the online version of the survey by a URL-embedded message in the text (Appendix B). The recipients were directed to click on the hypertext link, which evoked the researcher's Web browser. The participants completed the survey instrument utilized in the study by accessing the researcher's URL (available through the university's server). All students were encouraged to complete the survey within two weeks. A data log was maintained by the Delphi Center so that surveys returned by the student were assigned a response identification number for each respective course they were enrolled. This allowed students who may have been enrolled 
in more than one online course to respond to the survey based on their experience for each of their respective online courses.

To maximize response rate, students were sent a personal email message one week after the initial contact, again requesting participation with directions to the hypertext link. This methodology is supported by Schaefer and Dillman (1998) who claim that response rate increases through multiple contacts. Schaefer and Dillman further identified that a personalized email message is an important element in increasing the response rate as it makes the individual feel more important. To further increase response rate, students were sent a second reminder via a personalized email message two weeks following the first reminder and a third reminder five weeks later. This corresponds to Dillman's (1978) prescription that reminders be sent one, three, and seven weeks after the initial mailing of a survey. The timeline and tasks involved in data collection are shown in Table 3.

\section{Table 3}

Timeline and Tasks for Data Collection

\begin{tabular}{|l|l|}
\hline Timing & Tasks \\
\hline $\begin{array}{l}1.6^{\text {th }} \text { week of } \\
\text { semester. }\end{array}$ & $\begin{array}{l}\text { 1. Notify and inform course participants through email about the } \\
\text { research study and that they will receive an online survey. }\end{array}$ \\
\hline $\begin{array}{l}2.7^{\text {th }} \text { week of } \\
\text { semester. }\end{array}$ & $\begin{array}{l}\text { 2. Notify course participants through email of survey and include a } \\
\text { "quick link" to the online version of the instrument. }\end{array}$ \\
\hline $\begin{array}{l}3.8^{\text {th }} \\
\text { semeek of }\end{array}$ & $\begin{array}{l}\text { 3. Send participants first reminder through a personal email message } \\
\text { with directions to the hypertext link. }\end{array}$ \\
\hline $\begin{array}{l}\text { 4. } 10^{\text {th }} \text { week of } \\
\text { semester. }\end{array}$ & $\begin{array}{l}\text { 4. Send participants a second reminder via personalized email } \\
\text { message with directions to the hypertext link. }\end{array}$ \\
\hline $\begin{array}{l}5.14^{\text {th }} \text { week of } \\
\text { semester. }\end{array}$ & $\begin{array}{l}\text { 5. Send participants a third and final reminder via personalized email } \\
\text { message with directions to the hypertext link. }\end{array}$ \\
\hline $\begin{array}{l}\text { 6. Length of the } \\
\text { study. }\end{array}$ & $\begin{array}{l}\text { 6. Maintain a data log on surveys submitted and assign a course } \\
\text { response identification number for each returned survey. }\end{array}$ \\
\hline
\end{tabular}


As students submitted the survey, data were entered into a password protected website through the Delphi Center for Teaching and Learning that could only be accessed by the researcher. If a request was made by participating course faculty to receive the data from the study, it would only be shared in aggregate form to maintain the confidentiality of all participants and to report overall perception of student social presence, perceived quality of learning, perceived satisfaction, and intent for future enrollment with online courses.

Data Analysis

Because the data collected are both quantitative and qualitative in nature, different analysis for each research method was conducted. This study sought to establish the degree of relationships among students' perceptions of their satisfaction with their learning experience and social presence.

To answer the first research question, correlations were calculated between the perception of social presence and perceived satisfaction with learning with the online course. To answer the second research question, correlations were performed to examine the relationship between social presence, perceived quality of learning, and satisfaction with learning in course activity experiences. Moreover, based on theory and research to determine the order of variable entry, a hierarchical regression analysis was employed to find the unique variance explained by social presence and satisfaction with learning in predicting future enrollment in online courses.

All of the statistical analyses were carried out using a computer software package, SPSS version 14.0 Statistical Package for the Social Sciences. The quantitative data are 
presented in table form. The statistical procedures and analysis that were calculated for each research question are shown in Table 4.

\section{Table 4}

Statistical Analysis of Data

\begin{tabular}{|c|c|c|}
\hline Research Question & $\begin{array}{l}\text { Variable(s) } \\
\text { measured }\end{array}$ & $\begin{array}{l}\text { Statistical procedure(s) to } \\
\text { be used }\end{array}$ \\
\hline $\begin{array}{l}\text { 1. What is the relationship } \\
\text { between learners' } \\
\text { perceptions of social } \\
\text { presence and their } \\
\text { perceived satisfaction with } \\
\text { learning in online courses? }\end{array}$ & $\begin{array}{l}\text { Learners' perceptions of } \\
\text { social presence. } \\
\text { Perceived satisfaction with } \\
\text { learning in online courses. }\end{array}$ & Correlation. \\
\hline $\begin{array}{l}\text { 2. What is the relationship } \\
\text { between learners' } \\
\text { perception of social } \\
\text { presence, satisfaction with } \\
\text { and quality of learning in } \\
\text { online course activities? }\end{array}$ & $\begin{array}{l}\text { Learners' perception of } \\
\text { social presence in online } \\
\text { course activities. } \\
\text { Perceived satisfaction and } \\
\text { quality of learning in online } \\
\text { course activities. }\end{array}$ & Correlation. \\
\hline $\begin{array}{l}\text { 3. What is the relationship } \\
\text { between learners': (a) } \\
\text { perceptions of social } \\
\text { presence and perceived } \\
\text { satisfaction with learning in } \\
\text { online courses, and (b) } \\
\text { intention to enroll in future } \\
\text { online courses? }\end{array}$ & $\begin{array}{l}\text { Learners' perceptions of } \\
\text { social presence. } \\
\text { Perceived satisfaction with } \\
\text { learning in online courses. }\end{array}$ & $\begin{array}{l}\text { Correlation } \\
\text { Hierarchical regression. } \\
\text { Predictor variables: } \\
\text { Learners' perceptions of } \\
\text { social presence (entered } \\
\text { first). Perceived } \\
\text { satisfaction with learning in } \\
\text { online courses (entered } \\
\text { second). } \\
\text { Dependent variable: } \\
\text { Intention to enroll in future } \\
\text { courses. }\end{array}$ \\
\hline
\end{tabular}

Qualitative analysis was conducted on the participants' responses submitted from the open-form questions. This form of questioning provides insight not available through closed form survey items (Rossman \& Rallis, 1998). According to Rossman and Rallis, after gathering qualitative data, the researcher should generate categories, identify 
themes, and look for recurring patterns among the responses to the open form items. A code number was assigned for each identified category and recurring theme. The interpretation and summary of these items are included in the report of the results.

\section{Limitations}

There are several limitations to this research design. One is that the correlational method cannot establish a cause-effect relationship between variables that are correlated (Pagano, 1996). Other limitations include the lack of randomization, manipulation, and control that characterizes experimental studies. Since the participants in this study belong to an "intact group" and are administratively defined, as is the case in educational settings, these procedures were outside the control of the researcher (Gall et al., 1996). Because this was an exploratory study, the participants were drawn from a purposive group who had the Web-based online learning experience to provide the requested data.

Another major limitation of this study is that it only takes into consideration the perceptions of the students who respond to the survey. The viewpoints of the students who either did not respond or withdrew from the course before the end of the semester were not included in the data. The response rate to an Internet survey is another limitation. Electronic survey response rates tend to be lower than compared to mailed surveys. Low response rates may result in a biased estimate of the characteristics of the population (Bean \& Roszkowski, 1995). An additional limitation of this study is the lack of generalization of the results beyond students enrolled in the Web-based online courses at the institution participating in this study. Therefore, generalization should only be made about the targeted population for this study. However despite these limitations, the 
perceptions of students being examined may be beneficial to practitioners who are currently involved, considering, or researching online delivery courses.

The results of this study are presented in Chapter IV and discussion and recommendations for future research in this area are presented in Chanter V. To that end, the goal of this study is to contribute to the field of online education by bringing attention to the importance of developing and supporting online learning environments that are effective in the promotion of learning through the interaction and social presence that are taking place. 


\section{CHAPTER IV}

\section{RESULTS}

This study examined the perception of social presence and its influence on learning and retention in courses that were delivered entirely online. Correlations to determine the relationship were performed between students' overall social presence scores and students' overall perceived learning scores for an online course. In addition, correlations were performed to examine the relation between social presence and perceived quality of learning in course activity experiences. A hierarchical regression analysis was employed to find the unique variance explained by social presence and learning in predicting future enrollment in online courses. The 14.0 Statistical Package for the Social Sciences was used for all analyses, except for the qualitative data. The results from the open-ended questions were explored to help inform and support the findings of the quantitative data. The study was designed to answer the following three research questions:

1. What is the relationship between learners' perceptions of social presence and their perceived satisfaction with learning in online courses?

2. What is the relationship between learners' perceptions of social presence, satisfaction with learning experiences and quality of learning in online course activities, e.g., class discussions, group projects? 
3. What is the relationship between the perceptions of social presence, learning, and the likelihood of future enrollment in online courses?

In this chapter, the demographics of the participants are provided along with appropriate data presentation related to each of the three research questions. A discussion of the results will also be included.

\section{Demographic Variables}

Demographic information is provided for a description of the participants in terms of their age, gender, online course experience (number of courses), number of college credits, and reason for participating in an online course. A total of 280 participants enrolled in 39 online courses responded to the online questionnaire. This resulted in a $28.7 \%$ response rate of the $(N=975)$ students that were enrolled in the online courses. These response rates are consistent with previous research on Internet surveys (Crawford et al., 2001; Simsek \& Veiga, 2001).

Recent studies on social presence (Gunawardena \& Zittle, 1997; Tu, 2002) have indicated a need for an investigation of a diverse sample of course content, e.g., business, education, and psychology. Previous studies on social presence have focused primarily on graduate cohort participants enrolled in education or business courses (Gunawardena \& Zittle, 1997; Richardson, 2001; Shih, 2004; Tu, 2002). The authors further recommended that students be selected with individual differences on such variables as level of college credits, overall computer experience, and online learning experience. The online courses that served as a resource included both undergraduate $(n=21)$ and graduate level $(n=18)$ and involved various disciplines including communication $(n=$ $8)$, computer engineering and computer science $(n=2)$, education $(n=6)$, human 
resource development $(n=8)$, justice administration $(n=6)$, Pan-African studies $(n=3)$, psychology $(n=2)$, sociology $(n=3)$, and nursing $(n=1)$.

More than half of the participants were female $n=187(67.8 \%) ; 91$ males (32.5\%) comprised the remainder. Multiple age groups were represented with a range of 18 years to 57 years. The largest group represented was $25-35$ years of age (53.95\%), followed by under 25 years of age (30.69\%), 36-45 years of age (20.46\%), and 46 plus years of age $(13.48 \%)$, respectively. The mean age was $34.32(S D=9.1)$. These demographic characteristics are congruent with previous studies about distance learners that indicate females comprise at least $60 \%$ of the sample and the majority are adults between the ages of 25-50 (Hardy \& Boaz, 1997; Wolcott, 1996).

The number of online courses taken ranged from 5 courses to none. Nearly half of the participants had taken 1-3 online courses (42.8\%), closely followed by 4-5 (41.78\%), and a small percentage (15.35\%) had taken none. The mean for online course experience was $3.22(S D=1.7)$. The number of credit hours completed ranged from a minimum of 3 to a maximum total of 250 credit hours. Nearly three-quarters of the participants (72.96\%) indicated they had completed up to 120 credit hours at the undergraduate level with $47.4 \%$ of those at the junior/senior level. This was followed by those who were considered to be enrolled in graduate studies (26.66\%).

Participants were asked to select the reasons or their motive for taking the course in the online format. An itemized list of choices modified from the literature on distance education was developed for this purpose. Motives for pursuing distance education generally include physical distance from the institution, scheduling conflicts, and preference for distance education modes (Eastmond, 1995; Harasim, 1996). More than 
one reason could apply as a motive. The primary reasons selected by the participants were (1) they liked the convenience of taking courses online $(73.9 \%),(2)$ the course was required for their major (33.9\%), followed by (3) the category of other $(24.3 \%)$, or (4) the course was only offered online (19.6\%). The category of "other" was listed as an option to provide participants an opportunity to reveal additional factors they considered when selecting an online course delivery mode. Several of the reasons given were based primarily on the need for the learner to gain access to university courses independent of time and place due to job and/or family responsibilities. A listing of reasons cited is included in Appendix D .

The features of place-and time-independent communication seemed to appeal to many as expectations of job and family responsibilities as well as geographic distance were frequently cited by those who self-selected to take the course online. For example, the cost of education continues to increase and the student needs to seek online education in an attempt to balance the cost of education and the need for an income. In addition, when classroom courses were not available when a student needed them, students were increasingly turning to online courses to fill in the gaps or even complete their degrees. An additional percentage of the students $(9.3 \%)$ indicated they selected an online format because they simply enjoyed the online interaction with others. Further, $8.7 \%$ cited curiosity about the subject as the rationale for taking the online course. Curiosity is considered to be an important motivator of adult learning and it is thought to contribute to success in the development of lifelong learning and to be closely linked to self-directed learning (Candy, 1991; Reio, 1997). Tough (1969) discovered that satisfaction of 
curiosity was the second most commonly expressed reason for engaging in adult learning projects.

The typical participant in this study was 25 years of age or older, female, a junior/senior undergraduate, who possessed at least 3 online course experiences, and selected online courses for the convenience. These findings fit with the assertion in the distance education literature that online communication provides increased opportunities for learners who are unable to access traditional place-bound education and that most course participants will rate convenience as the primary reason for choosing an online mode of learning (Oliver, 1999; Woods \& Baker, 2004). A summary of the demographic information is presented in Table 5. 


\section{Table 5}

Demographic Information

Demographic

Categories

Frequency $\quad \%$ of Total

Age

$\begin{array}{crc}\text { Under } 25 & 66 & 30.7 \% \\ 25-35 & 116 & 53.9 \% \\ 36-45 & 44 & 20.5 \% \\ 46 \text { and up } & 29 & 13.5 \%\end{array}$

Gender

Female

Male

Online experience

None before this one

$$
1 \text { only }
$$

2-3

More than 3
187

91

43

62

58

117
$66.8 \%$

$32.5 \%$

College course credits

1-60 credits

70

$25.9 \%$

(fresh/soph)

61-120 credits

(junior/senior)

Over 120 credits

(graduate level)

128

$47.4 \%$

72

$26.7 \%$

Reasons for taking course

online

only offered online

like the convenience

55

207

required for major

95

enjoy interaction online

26

curiosity about the subject other*

25

68
$15.3 \%$

$22.1 \%$

$20.7 \%$

$41.8 \%$

* See Appendix D 
Survey Instrument: Students' overall perceptions of social presence, satisfaction with learning, and likelihood of future enrollment in an online course

Section Two of the questionnaire contained 44, six-point Likert scale items $(1=$ strongly disagree to $6=$ strongly agree) constructed to examine the research variables: students' perceptions of social presence, satisfaction with learning, and intent to enroll in a future online course. Of the 44 questions in this section, there were 23 items (items 123) selected from the combined and modified social presence scales (Gunawardena \& Zittle, 1997; Tu, 2002) constructed to assess students' overall perceived social presence and students' overall perceived satisfaction with learning in online courses.

In addition to the social presence questions in Section Two, respondents were to assess their satisfaction with learning experiences from five items that correspond to a Satisfaction with Learning Scale (items 24-28) derived from the Workplace Adaptation Questionnaire (Morton, 1993). An additional 12 items (29-40) prompted the student to indicate the value or importance they placed on social presence within the context of their Web-based course. The basis of these items included each attribute of the conceptual framework that relates to the construct of social presence as determined by the literature. These attributes included interaction, immediacy and intimacy (Argyle \& Dean, 1965; Gorham, 1988; Heath \& Bryant, 1992; Mehrabian, 1969; Moore, 1989; Short et al., 1976; Wagner, 1994, 1997; Wiener \& Mehrabian, 1968). The remaining 4 items (41-44) taken from the modified Intention to Turnover Scale (Abraham, 1999) were employed to prompt the student to indicate the likelihood of the student's intent to enroll or not in a future online course. 
Refer to Table 2 for the survey questions that refer to each construct pertaining to each of the research questions. For the full text of the survey questions, refer to the survey instrument (Appendix C ). Overall, the Cronbach's alphas were .92 for social presence, .95 for satisfaction with learning, and .84 for intent to enroll in future Webbased courses. The internal reliability of these scales is comparable to those reported in previous research studies (Abraham, 1999; Gunawardena \& Zittle, 1997; Morton, 1993; Reio, 1997; Tu, 2002). The Cronbach alphas for the attributes of social presence included .90 for immediacy, .92 for interaction and .81 for intimacy. Based upon the high correlations between the three measures of social presence (immediacy, interaction and intimacy), and the need to avoid multicollinearity issues (Gall et al., 1996), these variables were combined into one variable for statistical analysis purposes and labeled as “communication." The Cronbach's alpha for communication scale scores was .82 .

Because the items to measure intent to enroll in a future online course were modified from the Intent to Turnover Scale (Abraham, 1999), a principle component analysis (PCA) was conducted to test the measure's construct validity. The PCA results yielded but one principle component; the scree plot supported this interpretation as well. Thus, the research evidence suggests that the scale was measuring what it was designed to measure, i.e., intent to turnover.

What is the Relationship between Students' Overall Perception of Social Presence and Students' Overall Perceived Satisfaction with Learning?

To answer the first research question, correlations were calculated between overall social presence and overall perceived satisfaction with learning for the online course. The correlations were statistically significant at .01 level (2-tailed). All 
assumptions of normality, linearity, and homogeneity were met. The means, standard deviations and correlations among the variables are presented in Table 6.

\section{Table 6}

Means, Standard Deviations, and Correlations of Research Variables $(N=280)$

\begin{tabular}{|c|c|c|c|c|c|c|}
\hline Variable & $M$ & $S D$ & $\begin{array}{l}\text { Overall } \\
\text { social } \\
\text { presence }\end{array}$ & Communication & $\begin{array}{l}\text { Overall } \\
\text { satisfaction } \\
\text { with learning }\end{array}$ & $\begin{array}{l}\text { Intent to } \\
\text { enroll }\end{array}$ \\
\hline \multicolumn{7}{|l|}{ Overall Social } \\
\hline Presence & 98.00 & 18.04 & & & & \\
\hline Communication & 57.07 & 8.05 & $.59 * *$ & $-\ldots$ & & \\
\hline \multicolumn{7}{|c|}{ Overall Satisfaction } \\
\hline With Learning & 21.51 & 6.67 & $.72 * *$ & $.71 * *$ & --------- & \\
\hline $\begin{array}{l}\text { Intent to } \\
\text { Enroll in } \\
\text { Future }\end{array}$ & & & & & & \\
\hline Online & 20.11 & 4.87 & $.45^{* *}$ & $.40 * *$ & $.36^{* *}$ & --- \\
\hline
\end{tabular}

Note: $* * p<.01$

An examination of Table 6 shows that students' overall perceived satisfaction with learning yielded a correlation of .72 $(p<.001)$ with students' overall perceived social presence scores $\left(r^{2}=.52 ; p<.001\right)$ indicating a strong positive relationship between these two variables ("large" effect size, [Cohen, 1988]).

Because "communication" was associated with the attributes of social presence, the correlations between social presence and communication were examined as well. The results indicate that overall social presence and communication yielded a positive correlation of $.59(p<.001)$. Linear regressions were used subsequently to determine the 
amount of variance that either social presence or communication would explain in the satisfaction with learning variable. When entering the communication variable (a combination of immediacy, interaction, and intimacy), a significant prediction equation was established $(F=281.15 ; d f=287 ; p<.001)$. The $r^{2}$ of the equation was $.50(p<$ .001 ; large effect size). Thus, $50 \%$ of the variance in students' overall perceived satisfaction with learning was explained by the "communication" variable. Recent studies in the field of distance education and communication (Christophel, 1990; Gunawardena \& Zittle, 1997; McIsaac \& Gunawardena, 1996) have contended that immediacy, intimacy and interaction enhance social presence and play an important role in forming interpersonal relationships in the communication process. The strong, positive association of this scale's scores with students' overall perceived satisfaction with learning scores corresponds with the premise that a close interrelationship exists between satisfaction with learning and these communication cues.

Another linear regression was used to analyze the data further. See Table 6 above for the means, standard deviations, and correlations of the variables. The results suggest that a significant predictor equation was also established $(F=256.245 ; d f=235 ; p<.001)$ for social presence. The correlation between the two variables was .72, with an $r^{2}$ value of .52 $(p<.001$, large effect size $)$. Thus, $52 \%$ of the variance in students' overall perceived satisfaction with learning in their online courses is explained by students' overall perception of social presence. The strong, predictive relationship between the two variables implies that perceived social presence might be a significant factor contributing to perceived satisfaction with learning. This finding corresponds with the results of 
Gunawardena and Zittle's (1997) study that demonstrated that $60 \%$ of the variance in student satisfaction was contributed by social presence.

Social presence, satisfaction with learning and demographic variables

To further explore the relationship between perceived social presence and perceived satisfaction with learning, correlational analyses were conducted between the research variables of social presence and satisfaction with learning and the demographic information obtained from the survey. These items included age, gender, and online course experience. Previous research studies have indicated a relationship between overall social presence and these demographic variables (Arbaugh, 2000; Herring, 2000; Richardson, 2001; Shih, 2004). The means, standard deviations, and correlations between the demographic items and students' perceived social presence and satisfaction with learning are presented in Table 7. 


\section{Table 7}

Means, Standard Deviations, and Correlations for Demographic Items with Students' Perception of Social Presence and Students' Satisfaction with Learning

\begin{tabular}{|c|c|c|c|c|c|}
\hline Variable & $n$ & $M$ & $S D$ & $\begin{array}{l}\text { Correlations with } \\
\text { social presence }\end{array}$ & $\begin{array}{l}\text { Correlations with } \\
\text { satisfaction with } \\
\text { learning }\end{array}$ \\
\hline Age & 274 & 34.30 & 9.10 & .06 & .06 \\
\hline Gender & 277 & 1.67 & .47 & .02 & .04 \\
\hline Experience & 241 & 3.20 & 1.7 & .01 & .03 \\
\hline $\begin{array}{l}\text { Social } \\
\text { presence }\end{array}$ & 241 & 98.00 & 18.04 & $-\ldots$ & $.72 * *$ \\
\hline $\begin{array}{l}\text { Satisfaction } \\
\text { with } \\
\text { learning }\end{array}$ & 270 & 21.51 & 6.67 & $.72 * *$ & ---- \\
\hline
\end{tabular}

Note: $p<.001 * *$

An examination of Table 7 indicates that the correlations for students' perception of social presence, students' satisfaction with learning as well as the demographic items of age, gender, and online experience were not statistically significant $(p s>.05)$. This finding demonstrates that perceptions of social presence and perceptions of satisfaction with learning were not associated with age, gender, or amount of online experience in this study; thus, this result does not correspond with the literature that suggests that select demographics are related to social presence (e.g., Richardson, 2001). 
What is the Relationship between Students' Perception of Social Presence for Individual Course Activities and the Perceived Quality of Learning for those Activities?

To answer the second research question, students' perception of quality of learning and perception of social presence were examined in terms of the specific types of course activities that were provided in their online courses. The activities were divided among five categories based upon their usual occurrence in the courses. Section Three of the questionnaire contained four (items 1-4), six-point Likert scale items (1= strongly disagree to 6 strongly agree) designed to measure the quality of learning based on the variables of social presence, active involvement and collaboration (Hiltz et al., 2000). The Cronbach's alpha for the quality of learning scale scores was .93. Items 5-9 (based on a six-point Likert scale) in Section Three were items from the modified social presence scales of Gunawardena and Zittle (1997) and Tu (2002) that were used to measure the perception of social presence in the respective course activities (Cronbach's alpha $=.82)$.

A mean score for perception of social presence and quality of learning was computed individually for the five course activities. A high score on both scales indicated a learner perceived a higher degree of social presence and quality of learning for a particular course activity, respectively. The findings for the individual course activities are shown in Table 8. 


\section{Table 8}

Summary of Means and Correlations for Individual Course Activities

\begin{tabular}{|c|c|c|c|c|c|}
\hline Course activities & $n$ & $\begin{array}{l}\text { Mean score } \\
\text { for Social } \\
\text { Presence }\end{array}$ & $\begin{array}{l}\text { Mean score } \\
\text { for Quality } \\
\text { of Learning }\end{array}$ & $\begin{array}{l}\text { Correlation } \\
\text { between social } \\
\text { presence in } \\
\text { course activities } \\
\text { and quality of } \\
\text { learning }\end{array}$ & $\begin{array}{c}\text { Coefficient } \\
\text { of } \\
\text { determination } \\
\left(r^{2}\right)\end{array}$ \\
\hline Discussion & 273 & 20.44 & 19.18 & $.42 * *$ & $.18 * * *$ \\
\hline $\begin{array}{l}\text { Individual } \\
\text { projects }\end{array}$ & 271 & 15.47 & 20.02 & $.50 * *$ & $.25 * * *$ \\
\hline $\begin{array}{l}\text { Group } \\
\text { projects }\end{array}$ & 269 & 9.06 & 22.82 & $.35^{* *}$ & $.12 *$ \\
\hline $\begin{array}{l}\text { Reading } \\
\text { assignments }\end{array}$ & 268 & 16.88 & 19.18 & $.47 * *$ & $.22 * * *$ \\
\hline $\begin{array}{l}\text { Writing } \\
\text { assignments }\end{array}$ & 267 & 17.38 & 19.65 & $.40 * *$ & $.16^{* *}$ \\
\hline
\end{tabular}

Note: ${ }^{*} p<.05, * * p<.01, * * * p<.001$ (2-tailed)

An examination of the results indicates that the mean score for social presence in class discussions was $20.44(S D=9.42)$ and the mean score for perceived quality of learning was $19.18(S D=5.83)$. The highest mean for social presence was noted for the activity of class discussions. The statistical analysis yielded a correlation of $.42\left(r^{2}=.18\right.$; $p<.01)$. This suggests that social presence in class discussions accounted for approximately $18 \%$ of the variability ("medium" effect size, [Cohen, 1988]) in students' 
perceived quality of learning for this activity. Students with high perceived quality of learning also perceived a high social presence in class discussions.

Similar patterns of moderate to high relationships were observed with students in individual projects. The mean score for social presence for individual projects was 15.47 $(S D=9.44)$ and the mean score for perceived quality of learning was $20.02(S D=5.43)$. The statistical analysis yielded a correlation of $.50\left(r^{2}=.25 ; p<.01\right)$. This indicates that social presence in individual projects accounted for one-quarter of the variability (large effect size) in students' perceived quality of learning for this activity.

Both reading and writing assignments resulted in the highest means for perceived quality of learning. The mean score for social presence was $16.88(S D=8.40)$ and 19.18 $(S D=4.31)$ for learning in reading assignments. The statistical analysis yielded a correlation of $.47\left(r^{2}=.22 ; p<.01\right)$. This finding suggests that social presence accounts for $22 \%$ of the variability in perceived quality of learning for this course activity. The mean score for social presence in writing assignments was $17.38(S D=8.90)$ and for quality of learning the mean was $19.65(S D=4.70)$. A correlation of .40 between the variables $\left(r^{2}=.16 ; p<.01\right)$ was found. This indicates that social presence in written assignments accounted for $16 \%$ of the variability (medium effect size) in students' perceived quality of learning for this activity. What is interesting about these correlations is that although reading and writing are considered to be associated with individual activities, they had a moderate correlation and were positively related to social presence.

The lowest mean score reported for perceived social presence was for group projects. Respectively, the scores were $9.06(S D=11.07)$ for social presence and 22.82 $(S D=7.21)$ for quality. The relatively high standard deviation for the social presence 
variable in group projects suggests a great deal of variability in the variable, warranting careful interpretation. A correlation of $.35\left(r^{2}=.12 ; p<.01\right)$ between social presence and perceived quality of learning in group project activity indicates that $12 \%$ (medium effect size) of the variability in students' perceived quality of learning resulted from students' perceived social presence. The results suggest that social presence is a statistically significant predictor in perceived learning quality in group projects.

The fact that significant moderate to high correlations were demonstrated between perceived social presence and quality of learning for each of the five course activities indicates that social presence of either the instructor and/or other students was perceived to influence the quality of their learning experience. These results also suggest that social presence was important for even those activities that are generally considered to be an individualized activity. One possible explanation for this finding may be that students are expected or required to discuss their individual projects, writing and/or reading assignments with the instructor or other students prior to completing these activities. As a result, this may account for students' perception of social presence during these course activities.

To understand the learner's experiences and perceptions of learning in a computer-mediated course, correlations were calculated between the overall perception of social presence, overall satisfaction with learning, and the overall perception of quality of learning in all of the online course activities. The correlations were statistically significant at the $p<.01$ level (2-tailed). The correlation coefficients among the research variables of interest are presented in Table 9. 


\section{Table 9}

Summary of Correlations between Social Presence, Satisfaction with Learning, Quality of Learning and Course Activities

\begin{tabular}{|c|c|c|c|}
\hline Variable & $\begin{array}{l}\text { Overall social } \\
\text { Presence }\end{array}$ & $\begin{array}{l}\text { Overall satisfaction } \\
\text { with learning }\end{array}$ & $\begin{array}{l}\text { Overall quality of } \\
\text { learning }\end{array}$ \\
\hline \multicolumn{4}{|l|}{ Overall Social } \\
\hline Presence & $----\cdot$ & & \\
\hline $\begin{array}{l}\text { Overall Satisfaction } \\
\text { with Learning }\end{array}$ & $.72 * *$ & - - - - - & \\
\hline $\begin{array}{l}\text { Overall Quality of } \\
\text { Learning }\end{array}$ & $.55 * *$ & $.59 * *$ & - - - - \\
\hline \multicolumn{4}{|l|}{ Intent to } \\
\hline Enroll & $.45^{* *}$ & $.36^{* *}$ & $.42 * *$ \\
\hline
\end{tabular}

Note: $p<.01$ (2-tailed)

An examination of Table 9 shows that a positive correlation was found between the overall quality of learning and overall social presence scores $.55\left(r^{2}=.30 ; p<.01\right)$. The correlation between overall satisfaction with learning yielded a correlation of .59 ( $p$ $<.01)$ with students' perception of total quality of learning scores $\left(r^{2}=.35 ; p<.01\right)$ as well, indicating a strong, positive correlation between these two variables. These findings suggest that social presence explains $30 \%$ (large effect size) of the variance in the students' perception of quality learning in various course activities presented in an online environment. Thus, what is relevant to the purpose of this research is that these results may explain the importance of considering the social interaction dimension in computer- 
mediated learning environments when designing course activities in order to support learning.

What is the Relationship between Students' Overall Perceptions of Social Presence, Students' Overall Perceived Satisfaction with Learning, and Intent to Enroll in Future Online Courses?

To answer the third research question, correlations were calculated between the three main research variables of social presence, students' overall perception of satisfaction with learning, and intent to enroll in a future online course. The correlations were statistically significant at the $p<.01$ level (2-tailed). See Table 6 for the correlations among the research variables.

Through a review of the education, psychology, communication, and distance education literature, the evidence suggested that social presence would have a possible relationship with both satisfaction with learning and persistence in an online course, yet little empirical research had examined this relationship. In the first research question, there was a positive, strong relationship between all the measures of social presence, satisfaction with learning, and intent to enroll.

To test the conceptual model presented in this study where perceptions of social presence and perceptions of satisfaction with learning predicted intent to enroll in a future online course, a hierarchical regression analysis was conducted. The results in the Intent to Enroll Model are presented in Table 10. With social presence as the first block in the equation, $17.8 \%(p<.01)$ of the variance was explained in the regression equation (medium effect size; Cohen, 1988). The statistically significant beta weight was $(\beta=.33)$. 
After controlling for the social presence variable, the addition of overall satisfaction with learning in the second block of the regression equation resulted in an additional $1.1 \%(p=.05)$ of the variance being predicted in the regression equation; a small size effect (Cohen, 1988). The statistically significant beta weight was $(\beta=.14)$.

In essence, this suggests that perceived social presence is more of a contributing factor to the decision to enroll again in an online course than perceived satisfaction with learning. Overall, $18.3 \%$ of the variance in intent to enroll again in an online course was explained by the conceptual model (medium-large effect size). However, it should be noted that these results were obtained for one particular order of entry of the independent variables, based on the theoretical model.

\section{Table 10}

Hierarchical Regressions with Social Presence and Satisfaction with Learning Predicting Intent to Enroll in a Future Online Course $(N=285)$

Variable $\quad \beta \quad \Delta R^{2}$

Step 1

Social Presence

$.33 * *$

$.178^{*}$

Step 2

Satisfaction with Learning

$.14^{*}$

$.01^{*}$

Total $R^{2}$

$.18^{* *}$

Note: $* p=.05, * * p=<.01$. 


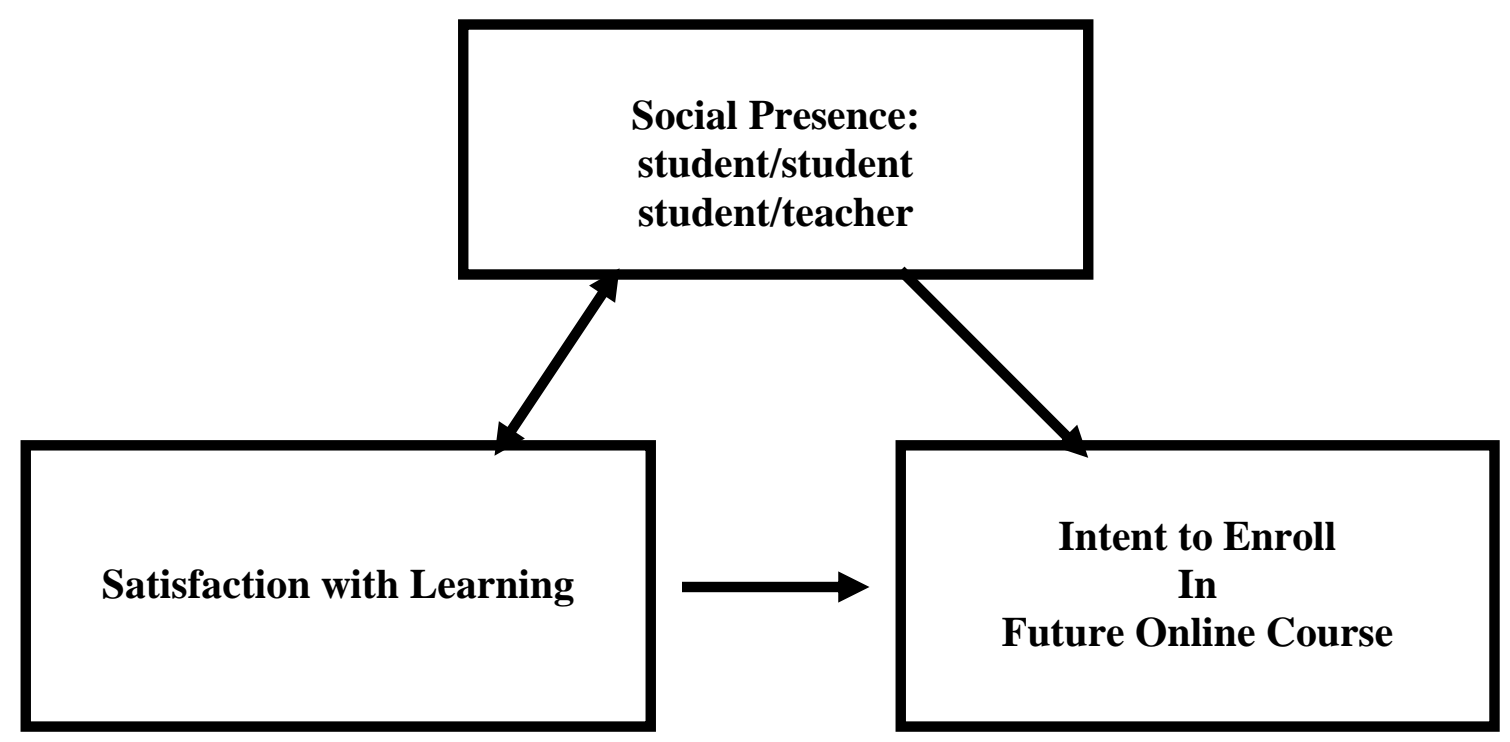

Figure 1. Intent to Enroll Model illustrating the influence social presence has on both satisfaction with learning and intent to enroll in a future online course. 


\section{Qualitative Data}

The qualitative part of the questionnaire, Section Four, was comprised of four open-ended questions that students answered pertaining to their perceptions of benefits, in terms of their quality of learning and satisfaction, related to the five course activities. Refer to Table 2 or Appendix C for these survey questions. The results from the openended questions were examined to provide further depth and breadth to the quantitative research findings.

Activities most beneficial to learning

The first question queried students about which activities they found most beneficial to their learning and why. Students had the option of entering more than one selection. Presented in Table 11 are the frequencies and percentages collected from students' indications of the activities they found most beneficial and the reasons for their selections are presented in Table 12. 


\section{Table 11}

Frequencies and Percentages of Students' Responses Regarding Beneficial Activities of Online Courses

\begin{tabular}{|c|c|c|}
\hline$\underline{\text { Activities }}$ & $\underline{\text { Frequencies }}$ & $\underline{\text { Percentages }}^{\mathrm{a}}$ \\
\hline Discussion & 99 & $33.50 \%$ \\
\hline Reading & 75 & $25.00 \%$ \\
\hline Writing & 56 & $18.60 \%$ \\
\hline Individual projects & 48 & $16.30 \%$ \\
\hline Group projects & 16 & $5.00 \%$ \\
\hline None & 2 & $.07 \%$ \\
\hline
\end{tabular}

${ }^{a}$ Students had the option of entering more than one selection; \% may not equal $100 \%$ 


\section{Table 12}

Frequencies and Percentages of Students' Responses Regarding Reason for Choosing Particular Course Activities as Most Beneficial

\begin{tabular}{|c|c|c|}
\hline Reason for selecting activity & Frequencies & Percentages $^{\underline{a}}$ \\
\hline
\end{tabular}

Increased knowledge, understanding

or application of material

96

$30.38 \%$

Instructor feedback/guidance/

facilitation

53

$16.70 \%$

Students' perspectives/feedback and/ or interaction

Clarification and/or reinforcement of material or expectations from others

Could work independently/did not have to rely on anyone else/selfpacing allowed

Participants' point of view acknowledged

$N=317^{\mathrm{a}}$

${ }^{a}$ Students had the option of entering more than one selection; \% may not equal $100 \%$ 
Discussion accounted for about one-third of students' responses as the most beneficial activity of their online course. Table 12 above, which lists students' reasons for indicating particular activities as beneficial to their learning, reports increased knowledge, interaction, feedback and other students' perspectives as a major reason for perceiving activities as beneficial. Further review and examination of the reasons students chose the activities indicate that an aggregate of $74 \%$ of participants identified interaction, feedback, guidance, clarification of material or expectations, and acknowledgement as their reasons for selecting the activities as beneficial. All of these reasons relate to the presence of others. This supports the quantitative findings that $97 \%$ of the students $(n=271)$ rated discussion higher for social presence than any other activity.

The correlation between social presence and their perceived quality of learning $\left(r^{2}=.26 ; p<.01\right)$ for this activity seems to reflect the strong value students place on the presence of others as being beneficial to their learning experience. Learners can interact while working in a learning activity and can see a problem from different perspectives. This can generate new solutions to problems through shared understanding. Those students who participated in class discussions indicated this not only helped them better understand the course materials, but also broadened their points of view, and helped them to become more receptive to different ideas. An example of this was stated by one student:

Group discussions obviously facilitated online communication and interaction, as there were specific questions to answer, corollary questions raised by the instructor or other students, and points of views different from my own to think about. 
Although individual projects, reading, and writing assignments are considered to be an independent activity, these activities also demonstrated significant correlations as presented in Table 8 between social presence and perceived quality of learning. These assignments are the activities that provide opportunities for students to apply the knowledge of the material and demonstrate their understanding of the content presented. Examples were evident from the students' comments.

Discussing what we have read in class reinforces what we have learned via the readings. Applying what we have read and then discussing it with others in class reinforces what we have learned through the assignment.

Reading and writing assignments because the writing assignments required an explanation related to comprehension of what was read and we had to interact with others related to the material read.

The individual projects were the most beneficial because you had to discuss them with others and you always learned something you didn't know from someone else and you had to be creative with how you applied the information.

These assignments are also the activities in which students may receive feedback or clarification from their instructors or other students as to the degree of learning or understanding of a topic or concept.

Reading assignments provided guidance in current theory but class discussions provided a forum to clarify concepts which were previously elusive.

Reading assignments were where I learned the material, and class discussions helped me understand the material.

Moreover, these activities provide opportunities for reflection and critical thinking with other students as illustrated by the following comments:

The readings sparked great debate and introduced me to a new way of looking at the content though the eyes of my cyber classmates.

We had to read an assignment and reflect on it on a discussion board for everyone to $\mathrm{read} /$ comment on. 
The writing assignments forced one to blend class discussions and reading into a synthesis

As group projects only represented 5\% of the online course activities in which students engaged, it was an interesting outcome that the analysis of the correlations between social presence and perceived quality of learning still yielded a positive, yet modest, correlation of $.35\left(r^{2}=.12 ; p<.01\right)$. This finding implies that students regard collaboration and interaction as beneficial to their learning even though they may not perceive this course activity as particularly beneficial.

Group projects for an online class usually do not work. It is difficult to meet because most people that take online classes have full-time employment or other classes. It's too hard to coordinate everyone's schedule. The group had to get together and help each other. You learn to rely on others and also be responsible with your own work. It was great to be able to rely on them to get their job done and know that they trust me to get my work done...it was a lot of fun interacting with them.

Group projects may provide a collaborative learning environment that allows students to engage in a process of exchanging ideas and diverse viewpoints and increases their interest while promoting their critical thinking. Students reported that the group assignments helped to provide a better understanding of the course concepts and application of their knowledge.

The group assignments facilitated learner-learner interaction. I felt that I learned a great deal from my other classmates. The learner-learner interaction helped clarify concepts.

The group project allows us to use what we have learned throughout the semester.

\section{Students' perceptions of interaction with the instructor}

Table 13 provides the frequencies and percentages collected from an open-ended question that queried the students about how much interaction they had with their instructor (e.g., significant, moderate, sufficient, or lacking). Approximately $74 \%$ of the 
participants $(n=179)$ indicated they had sufficient to significant communication with their instructor. The literature suggests that greater perceptions of social presence tend to be linked with greater perceived learning, course satisfaction, and satisfaction with the instructor (Gorham, 1988; Gunawardena, 1995; Gunawardena \& Zittle, 1997; Richardson \& Swan, 2003; Walther, 1992). The perception of interaction with the instructor in this study appears to indicate that the perceived "presence" of the instructor is considered to be an important contribution to students' perceived satisfaction and quality of learning. One reason may be due to the fact that the students perceive they are part of a learning community although they do not participate in face-to-face interaction. One student was able to describe the difference instructor "presence" made in contributing to their own satisfaction.

The instructor in particular is awesome. This is my second course with him online. I have taken other courses in other departments online, and I ended the semester feeling frustrated because of lack of communication on behalf of the instructor. The instructor is a great learning facilitator and the learning community not only helps me learn, but makes me feel as if I am a part of something.

It may be necessary for an instructor to have frequent and personal interaction with their students to develop satisfactory social presence, as was evidenced from one student:

Our instructor has special time set aside to answer our questions at the beginning of class, as well as at the end about assignments, etc. She is prompt in answering my emails. She makes me feel comfortable to talk to her.

Prompt feedback is also important for the students to develop a sense of "presence" of their instructor and to contribute to their satisfaction of learning. The distance education literature suggests that the role of the instructor should shift to a facilitator who fosters knowledge construction among learners (Garrison et al.,2001) in 
an online course. Some of the comments, however, reflected that select students still

looked upon the instructor as an authoritative figure and prioritized their interaction with the instructor before interaction with their peers. These findings may indicate that these students attributed their learning to interaction with their instructors and not with their peers. As a result, their perceptions of learning did not extend beyond the content level.

This instructor provides specific and significant responses and feedback to my discussion posts and to my questions. I am extremely satisfied with his level of involvement.

The only communication that I feel has truly been essential to my experience is feedback from my instructor. I have significant interaction with my instructor and she always gives me very good comments about how I could do better or how nicely written something was.

I learn much more from a professor than from another student. Although interaction is encouraged, it is not mandatory so it really didn't help much. The instructor always gave me feedback on assignments.

For some students, interaction with the instructor was often an exercise in frustration and, as a result, may have left the students feeling alone, disconnected, and dissatisfied with the course.

I have never gotten comments back on any of my submitted work (papers or whatever). I am used to getting feedback on work that is turned in and I feel that the lack of it is a detriment.

I don't believe that online communication was facilitated during this course. I had very little interaction with the class instructor. I wish there had been more feedback from the instructor or possibly some class notes or materials online. I never knew if I was actually learning the material.

The online course was less than mediocre, less than I expected. I had little to no communication with my instructor. I contributed the least learner-learner interaction of my group.

These findings support the quantitative results in this study that indicated students' overall satisfaction with learning yielded a strong, positive correlation 
$(.72 ; p<.01)$ with students' perceived social presence and $.71(p<.01)$ with "communication" (interaction, immediacy, and intimacy). The literature on teacher immediacy behaviors has found close and positive relationships between instructor immediacy and students' ratings of learning and instruction (Hornik \& Johnson, 2003; Richardson \& Swan, 2003). The previous comments indicate the importance of a close instructor-student relationship in the success of online learning.

The comments from the participants indicate that it is important to understand how the function of social presence can make the nature of online learning more interactive, appealing and engaging leading to an increase in academic integration and a successful learning experience for the learner.

\section{Table 13}

Frequencies and Percentages of Students' Responses regarding Amount in Interaction with Instructor

$\underline{\text { Amount of interaction } \quad \text { Frequencies } \quad \underline{\text { Percentages }}}$

\begin{tabular}{lcc}
\hline Significant & 58 & $23.90 \%$ \\
Moderate & 62 & $25.60 \%$ \\
Sufficient & 59 & $24.40 \%$ \\
Lacking & 63 & $26.00 \%$ \\
$\quad$ Totals & 242 & $100.00 \%$ \\
\hline$N=242$ & &
\end{tabular}


Perceptions of learner-learner interaction

An additional open-ended question queried students as to whether they thought the type and amount of learner-learner interaction was adequate for the course. The constructivist theory advocates collaborative learning as a process of knowledge construction on the part of individual students (Kanuka \& Anderson, 1998). The notion of using community as an ideal place for learning is an important issue in distance learning because it can generate a positive learning experience (Eastmond, 1995) and it can affect student satisfaction, retention and performance (Misanchuk, Anderson, Craner, Eddy, \& Smith, 2000; Neo, 2003).

Similar to the results found with their perception of interaction with the instructor, the majority $(70 \%)$ of participants indicated they felt interaction with other students was adequate. Generally, these findings support the quantitative results demonstrating that students' perceived social presence from others (including both peers and instructors) was closely related to their perceived satisfaction with learning in an asynchronous online environment.

From the following comments, it suggests that students' perceived social presence from their peers and their perceived social presence from their instructor may have minimized the psychological distance and influenced students' perceived interaction and learning from the online course activities.

With the online discussions we were able to get to know each other and at the start of each class we talked about the same things that I talk about in my classes on a college campus. I believe that my instructor had appropriate interaction. She replied to emails and led the discussions. She seemed to know when to lead and when to sit back and let us bounce off ideas with each other. 
Class discussions are so important in online courses. I have two other courses that do not offer this option and I feel that I do not gain a sense of community in these courses...I feel disconnected.

Without course activities like class discussions and assignments, there would be little communication and interaction online. Without these, it is easy to become isolated.

Learning from others' experiences is very helpful. It is a great support system as well. I have found that I was able to have a system of support, even though the students and instructors are not meeting face-to-face. The students email each other to help each other.

This was my first online course and I was a little concerned that it would be a sterile experience but I felt that I really got to know my classmates and instructor.

Intent to enroll in a future Web-based course

Of particular interest to this study is how online learners' perceptions of social presence and their perceptions of satisfaction with learning were related to their likelihood of future enrollment in another online course. An examination of the results generated from the question that inquired if the student would consider taking another Web-based course as a result of their experience with the present course they were enrolled informed and supported the quantitative data. Corresponding with the quantitative results, the qualitative data also illustrated that students with differing perceptions of social presence had influenced their decision to enroll in a future Webbased course.

Of the participants $(n=232)$ who responded to this question, nearly $75 \%$ $(n=173)$ responded in the affirmative as opposed to $12 \%(n=28)$ who responded they would not enroll in another online course. The analysis from those who responded to this question negatively indicated their dissatisfaction with the course design and the lack of interaction or sense of "presence" they had with their peers and the instructor. 
I did not get a lot of learner-learner interaction in the course and the instructor did not respond to my problems. I will not enroll in another online class.

I did not enjoy it. I prefer classroom interaction. I learn better with face-to-face interaction so I would only take another online class if it was the only way it was offered.

I did not like taking the online class. I felt like I did not benefit from it. I would rather go to class two or three days a week. I really had trouble learning and understanding the material. If I could go back and re-register for this class, I would take it during the day on campus.

One interesting result that was determined from the responses to this question was that the remaining $13 \%(n=31)$ reported they would enroll in another online course despite the poor satisfaction with learning they had experienced within their present asynchronous course. Various explanations were provided as to the rationale for this decision that ranged from the need to finish a degree program that was only offered online to positive experiences either they had previously or that were related to them by other students who had taken courses online. The following statements illustrate this:

I have to take online classes based on where I live and my occupation, but I don't like them. Communication and interaction is limited. I am happy to have this opportunity to complete my degree and I will accomplish this however I can. If time permitted, I would be in class and taking traditional classes for the social benefit. Unfortunately, online is the only way I can do this.

I would consider taking another web-based course, but not because of my experience in this course, I have taken three other online classes and had really good results. There was always lots of feedback and had quick responses to questions. I felt that the other instructors understood the need to communicate and build their discussion forums around the course content. I have enjoyed my past online experiences....however; I was not impressed with this course.

I will consider taking another online course only because of hearing other student experiences that were much more positive than this online course.

If I only had this experience to base my decision on, I would probably never take another online course again. However, I know several other students that have had positive experiences. 
Additionally, the analyses of the qualitative data revealed that the students who perceived positive social presence of their instructors and other learners and who also experienced active interaction with them were all satisfied with their learning. In fact, several students preferred the learning experience online rather than the traditional oncampus classes. These findings correspond with results from previous studies that suggest that the perceived presence of others, including both instructors and students, was closely related to satisfaction with online learning (Gunawardena \& Zittle, 1997; Richardson \& Swan, 2003).

I felt the instructor was extremely efficient in responding to any question I had and always gave me a satisfactory answer. He showed concern and support with the discussion board assignments, projects, and provided test review sheets. The amount of information I learned from this class exceeded my wildest expectations.

I feel that I have learned and contributed more in this online class than others prior, even classes on campus. I feel that my instructor has set up a near perfect method of facilitating an online class. I appreciate the hard work from the instructor and the caring attitude.

This was my first online course and now I am not afraid like I was before taking this course. I feel that I know the students better in this class than many oncampus classes.

I was apprehensive about taking an online class, but this class has shown me than an online education can be a good one.

\section{Summary}

According to the theoretical literature, it has been concluded that social presence exerts significant influence upon improving instructional effectiveness ( Tu \& Corry, 2001); therefore, the amount and quality of social presence might play a strong role in the satisfaction and motivation students derive from an online course. The results of this research suggested a strong, positive relationship between perceived social presence and 
satisfaction with learning. Furthermore, the correlation between students' perceived social presence and perceived satisfaction with learning resulted in a statistically significant relationship with students' intent to enroll in a future online course. The hierarchical regression analysis demonstrated that students' perceived social presence and perceived satisfaction with learning were indicators of their plans to enroll in future online courses. However, social presence was associated as having the most influence on intent to enroll in future online courses. Thus, the decision to persist with enrollment in asynchronous Web-based courses was most likely influenced through the mediation of the social presence variable for the participants in this study.

In addition, the analyses of the qualitative data from this study suggests that students who perceived a higher social presence from others also positively influenced their perceptions of learning, their satisfaction with online courses, and their motivation to enroll in Web-based courses in the future. The relevance of these findings suggests that students' perceptions of social presence is an effective indicator of their satisfaction with learning and intent to enroll in a computer-mediated environment, particularly in courses or programs that are totally online.

Chapter V will present the implications of this study, recommendations for future research, and make conclusions. 


\section{CHAPTER V}

\section{DISCUSSION}

Overview

Researchers often infer social presence as a key feature in the development of a learning community and as an important factor in the success of online learning in its ability to support cognitive learning by making interaction engaging and rewarding (Harasim et al., 1995; Gunawardena, 1995; Gunawardena \& Zittle, 1997, Rourke et al., 2001). Social presence involves the ability of people to be perceived as real, despite not communicating face-to-face (Garrison et al., 2000; Gunawardena, 1995; Short et al., 1976; Tu \& McIsaac, 2002). Of the empirical evidence that does exist, very little of it examines the aspects and/or benefits of social presence in online learning, particularly in courses or programs of study that are totally online. Moreover, the body of distance education literature is lacking studies investigating the construct of social presence, the value placed on it by online learners, and whether its existence in text-based environments is necessary for learning, satisfaction, and contributing to course retention.

The purpose of this study was to examine the role of social presence and its influence on satisfaction with learning and retention/continued enrollment in asynchronous learning environments utilizing an online survey administered to students $(n=280)$ enrolled in online courses of various disciplines, both undergraduate and graduate level. More specifically, it examined the relationship between students' perceptions of social presence and students' perceived satisfaction with learning in online 
courses. This relationship was also examined between perceptions of social presence in individual course activities and perceived quality of learning for those activities.

Furthermore, this study examined whether social presence could be used in predicting students' likelihood of future enrollment in online courses.

The following research questions were used in the design of this study:

1. What is the relationship between learners' perceptions of social presence and their perceived satisfaction with learning in online courses?

2. What is the relationship between learners' perceptions of social presence, satisfaction with learning experiences and quality of learning in online course activities, e.g., class discussions, group projects?

3. What is the relationship between the perceptions of social presence, satisfaction with learning, and the likelihood of future enrollment in online courses?

The results of the analyses of quantitative and qualitative data presented in the previous chapter illustrated the role of social presence in asynchronous online courses in light of these three research questions. The results suggested that students' perceived social presence was significantly and positively related to their overall perceived satisfaction with learning in online courses. Furthermore, significant moderate to high correlations were demonstrated between perceived social presence and perceived quality of learning for each of the five course activities examined in this study. A conceptual model was tested by conducting a hierarchical regression analysis to find whether perceptions of social presence and perceptions of satisfaction with learning influence the intent to enroll in future online courses. The results suggested that after perceived social 
predicts intent to enroll in an online course, perceived satisfaction with learning predicts only a small amount of additional variance.

Based on the results of this study, this chapter will discuss the findings in relation to the literature and the potential contribution to the theory of socio-cultural learning, the potential implications for practice for online course designers and instructors, the limitations of this study, and recommendations for future research. The following sections provide discussions of each predictive relationship through the presentation of findings from both the quantitative and the qualitative data.

Relationship between Students' Perceptions of Social Presence and Perceived Satisfaction with Learning in Online Courses

With regards to the first research question, the quantitative results from this study corresponded with results from previous studies that demonstrated a strong, positive relationship between the two variables of perceived social presence and perceived satisfaction with learning (Gunawardena \& Zittle, 1997; Richardson \& Swan, 2003). The correlation between these two variables yielded a strong, positive, statistically significant result of $.72(p<.01)$. The linear regression indicated that students' overall perception of social presence accounted for $52 \%$ of the variance ("large" effect size, [Cohen, 1988]) in students' overall perceived satisfaction with learning in their online course. The strong predictive relationship between these two variables implies that perceived social presence might be a significant factor contributing to perceived satisfaction with learning.

The quantitative analysis of social presence between the variables of "communication" (a combination of immediacy, interaction, and intimacy) and students' overall perceived satisfaction with learning also demonstrated a strong, positive 
correlation of .71 $(p<.01)$. A linear regression determined that the "communication" variable alone accounted for 50\% of the variance ("large" effect size, [Cohen, 1988]) in students' perceived satisfaction with learning. The strong, positive relationship of this scale's scores with students' overall perceived satisfaction with learning scores corresponds with the premise from the distance education literature that a close interrelationship exists between satisfaction with learning and the communication cues that have been attributed to enhancing social presence and forming interpersonal relationships in the communication process (Christophel, 1990; Gunawardena \& Zittle, 1997; McIsaac \& Gunawardena, 1996). The results suggest that the development of social presence and the ability to interact in active collaborative learning seems clearly to matter to those participants involved in this study.

Correlation analyses were also conducted between the research variables of social presence and satisfaction with learning and the demographic information (age, gender, and online course experience) obtained from the survey in this study. Although previous research studies have indicated a significant relationship between overall social presence and these demographic variables (Arbaugh, 2000; Herring, 2000; Richardson, 2001; Shih, 2004), the results of this study indicated that the correlations were not statistically significant $(p s>.05)$. This finding demonstrates that perceptions of social presence and perceptions of satisfaction with learning were not associated with age, gender, or amount of online experience in this study; thus, this result does not correspond with the literature that suggests that select demographic variables are related to social presence (e.g., Richardson, 2001). This interesting finding warrants future research. 
Students' Perception of Social Presence in Individual Course Activities and Relationship to Perceived Quality of Learning

With regards to the second research question, significant moderate to high positive correlations were demonstrated between perceived social presence and perceived quality of learning for each of the five course activities students were asked to evaluate. This indicates that social presence of either the instructor and/or other students was perceived to influence the quality of their learning experience. However, the nature of the assignments in the course may have been responsible for the relationship between perceived social presence and perceived quality of learning as those assignments that feature interaction with other students would be presumed to benefit from greater levels of social presence.

As expected, class discussion resulted in the highest perception of social presence as well as perceived quality of learning as this activity generally allows for interpersonal interaction. Students commented that participation in class discussions helped them better understand the course materials, broadened their points of view, and helped them become more receptive to different ideas.

Despite the fact that the activity of group projects would be expected to demonstrate interaction and perhaps high perceived social presence and quality of learning, this study resulted in a positive, but modest, statistically significant relationship between these variables. The relatively high standard deviation for social presence scores suggests that a great deal of variability in this variable exists, which means that this result should be interpreted with caution. However, $12 \%$ of the variability in students' perceived quality of learning could be explained in students' perceived social presence. 
Thus, the results suggest that social presence is a statistically significant predictor of perceived learning quality in group projects.

One reason students may not have considered group projects to be beneficial to their learning is that many students indicated they felt that group projects do not work well in an online environment due to the difficulty of coordinating everyone's schedule and relying on others to complete the assignment. However when students were encouraged to accomplish their learning tasks through group work, they regarded collaboration and interaction as beneficial to their learning as they reported that the group assignments helped to provide a better understanding of the course concepts and application of their knowledge.

Perhaps what is more interesting from the results of this study is the finding that moderate to high positive correlations were demonstrated between perceived social presence and quality of learning even for those activities that are generally considered to be an individualized activity, such as individual projects, reading and writing assignments. It was discovered through the qualitative analysis that students were expected to discuss these assignments with the instructor or other students prior to completing these assignments. The feedback, reinforcement, and exchange of ideas and resources that were involved in these discussions helped to support learning objectives and raise the level of social presence. This also enabled students to apply the knowledge of the material and demonstrate their understanding of the content that was presented.

Despite the support for the constructivist and collaborative approaches to learning, the role of the instructor appeared to be the primary factor in the development of social climate within the course activities as conventional pedagogy was still utilized in 
some of the online courses. Garrison et al. (2000) defined the instructor's role in online education as that of a facilitator and moderator that fosters interaction and collaboration among learners. From the students' comments to the open-ended questions, it appeared that the lecturing mode and moderating strategy of the instructor in some courses urged students to focus on the learning assignments, thereby, causing a decrease in social interaction. In these cases (based on the qualitative data), only a purely lecture or notebased online activity provided a high level of learner-content interaction, a moderate level of learner-instructor interaction, and little to no formal learner-learner interaction. These findings indicate that social presence may either grow or vanish as a function of the moderation or intervention approach of the instructor.

Students reported they regarded interaction and collaboration as beneficial to their learning. Corresponding to social constructivist learning theory, this study has shown that when students perceive higher social presence from both their peers and instructors they tend to perceive that they learn more (quality) from interacting with others than from reading the materials or interacting with the content alone (Kanuka \& Anderson, 1998; Vygotsky, 1978).

Thus, these results may account for the fact that students' perception of social presence during these course activities contributed to their experiencing learning through collaboration rather than a solitary process and tended to feel more satisfied with the quality of their learning. Furthermore, these results lend empirical support to research studies that indicate raising social presence in online environments may help to create perceptions of quality related to the experience on the part of the learner (Newberry, 2001). 
To better understand the learner's experiences and perceptions of learning in a computer-mediated course, correlations were calculated between the overall perception of social presence, overall satisfaction with learning, and the overall perception of quality of learning in all of the online course activities. Correlations were statistically significant at the $p<.01$ level (2-tailed) resulting in social presence explaining $52 \%$ of the variance in student satisfaction with learning and $30 \%$ of the variance in perception of quality learning in various course activities in an online environment. These results may explain that social presence served as a significant factor in all of the course activities and was perceived as an essential aspect of the students' educational experience.

The results of this study concur with the educational and communication literature which states that interaction is a main factor behind creating a high feeling of social presence (Wegerif, 1998), and learning is an interactive process in which the learners actively construct knowledge and then build upon that knowledge through the exchange of ideas with others and the responses/feedback of others (Harasim, 1990; Vygotsky, 1978). Furthermore, these results imply that the nature or content of the online course activities impacted the levels of interaction, participation, and collaboration that took place. Thus, it is important that developers, designers, and course instructors of online courses take appropriate measures to incorporate activities that will contribute to improved engagement and collaboration between learner-learner and learner-instructor; possibly leading to improved satisfaction and quality of learning.

Perceptions of Social Presence and Interaction with Instructors and Students and Satisfaction with Learning. In further exploration of the second research question, the literature on quality issues in distance learning (Phipps et al., 1998; Swan et al., 2000) 
suggests that data on measures of interaction and presence should be used in studying student performance and satisfaction. The level of interaction, according to Muirhead (2001), has an impact on the quality of computer-mediated instruction. Moreover, the literature suggests that greater perceptions of social presence tend to be linked with greater perceived learning, course satisfaction, and satisfaction with the instructor (Gorham, 1988; Gunawardena, 1995; Gunawardena \& Zittle, 1997; Richardson \& Swan, 2003; Walther, 1992).

Based on the results previously discussed and the analysis of the open-ended questions that queried students about their interaction with the instructor and other students, the perception of interaction with the instructor and others in this study appears to suggest a direct relationship between perceived social presence and satisfaction with the instructor and the course. In particular, the perceived "presence" of the instructor was considered to be an important influence in students' perceived quality of learning. From the comments, it was evident that the students who perceived positive social presence from their instructors and experienced active interaction with them were all quite satisfied with both their instructor and the course.

Likewise, comments from those students who experienced little social presence and interaction with their instructor indicated dissatisfaction with both the instructor and the course. Those instructors who failed to consider the relational dynamics in the online course setting seemed to produce greater feelings of isolation and reduced levels of student satisfaction. These findings correspond to a similar study (Richardson, 2001) that found students' overall perceived social presence accounted for $35 \%$ of the variability in students' overall satisfaction with the instructor. 
Research on teacher immediacy behaviors has found close and positive relationships between instructor immediacy and students' ratings of learning and instruction (Brady \& Bedient, 2003; Hornik \& Johnson, 2003; Richardson \& Swan, 2003). Immediacy is seen as a benefit of interactive learner-instructor communication since active ongoing communication is likely to result in an increased feeling of psychological closeness between the learner and the instructor. Similarly, the presence of such immediacy is likely to promote increased levels of interaction because learners and instructors are developing both a safe and rich interpersonal environment and a sense of connection or community as was evident from several students' comments.

The results from this study add to the importance of a close instructor-learner relationship in the success of online learning and the need for instructors and course designers to distinguish between the mere presence of a dyadic communication and the presence of genuine interpersonal interaction as they seek to improve the online educational experience. For example, the analysis of the qualitative data, in some instances, seemed to reflect the expectations students had of the instructor's role as an authoritative figure and prioritized their interaction with the instructor before interaction with their peers. These students attributed their learning to interaction with their instructors and not their peers and, as a result, did not develop a connection with other learners. Although individualized instructor-learner interactions may foster the development of a close learner-instructor relationship, it appears from the findings in this study that students may require the facilitation from the instructor to stimulate and sustain interaction among other learners. 
Similar to the results found with the perception of interaction with their instructor, the quantitative and qualitative results revealed that students who perceived more interaction with other students and higher social presence also perceived greater satisfaction with learning. Therefore, it is plausible to infer that students' perceptions of the interactions with other students may have influenced their social presence and perceptions of connection with others in the online learning environment. This reiterates the inseparability of learning from social dynamics and the endorsement of the creation of a sense of community.

The aforementioned results indicate that students' perceptions of social presence were closely related to the interactions they experienced in online course activities and thus contributed to their perceived satisfaction with learning. The results of the quantitative analysis found significant positive correlations among perceived social presence, perceived satisfaction, and quality of learning suggesting moderate to strong relationships among these variables. Qualitatively, the results of the open-ended questions indicated that students with different perceptions of social presence had different experiences with interactions, satisfaction with instructors, and perceived quality of learning.

The results of this research concur with the literature on social presence that emphasizes the importance of perceptions of the presence of others to the success of the online learning experience. Social interaction augments the relationship between learnerinstructor and learner-learner in the computer-mediated environment. Consequently, successful online course instructors and designers must face the challenge of finding ways to connect with students and for students to connect with other students in 
meaningful ways. This may be accomplished by developing interaction skills that foster a sense of social presence.

\section{Relationship between Perceptions of Social Presence, Satisfaction with Learning, and}

\section{Intent to Enroll or Persist in an Online Course}

Many issues are cited in the literature that may cause students undertaking online courses to reconsider their enrollment. The issue of isolation is an important factor that

can influence a student's attitude to online learning (Daugherty \& Funke, 1998; Wegerif, 1998; Woods \& Ebersole, 2003). This sense of isolation may make the difference between a successful and an unsuccessful online environment for many students.

In answering the third research question, the analyses of the quantitative results indicated that the correlations between the three main research variables of social presence, students' overall perception of satisfaction with learning, and intent to enroll in a future online course were statistically significant at the $p<.01$ level (2-tailed) indicating strong and positive relationships between all variables. These results suggested that perceived social presence would influence perceptions of satisfaction with learning and intent to enroll in a future online course.

Further investigation of the possible influence of either social presence or satisfaction with learning on the intent to enroll (dependent variable) was done. A hierarchical regression analysis resulted in social presence predicting $17.8 \%(p<.01)$ of the variance on the intent to enroll ("medium" effect size; [Cohen, 1998]) while overall satisfaction with learning explained an additional $1.1 \%(p=.05)$ of the variance ("small" effect size; [Cohen, 1998]) in the dependent variable. The relationship between social presence and persistence or intent to enroll in online courses seems relevant because the 
patterns of correlations between both of these measures achieved statistical significance. Likewise, the hierarchical regression suggests that the perception of social presence is more of a contributing factor to the decision to enroll again in an online course than perceived satisfaction with learning. Overall, $18.3 \%$ of the variance ("medium" effect size; [Cohen, 1988]) in intent to enroll again in an online course was explained by the conceptual model presented in this study (Table 10; Figure 1). Consequently, the decision to persist with enrollment in future asynchronous Web-based courses was most likely influenced by the social presence variable for participants in this study.

The significance of these results is supported in the literature that cites the importance of social presence fostering cognitive processes, strengthening interaction and collaboration, thereby, reducing the feeling of isolation and improving the satisfaction of students with online course offerings (Daugherty \& Funke, 1998; Evard, Churchill \& Bly, 2001; Wegerif, 1998; Woods \& Ebersole, 2003). Although it may not be true in every case, the data suggests that if learners are satisfied with the quality of their online education based on a positive social dynamic in parallel with the content delivery, then this may serve as a primary motivator to persist with enrollment. Conversely, without the existence of social presence and the behaviors it induces from students, satisfaction with online learning may be less assured and the persistence and retention rates in the online setting may suffer.

In sum, notwithstanding, there remains the need for additional research and development to explore this conceptual model further. 
Relationship between Social Presence, Constructivist Theory, and Community of Learning

A collaborative learning environment allows students to engage in a process of exchanging ideas and diverse viewpoints that increases their interest while promoting their critical thinking (Gokhale, 1995). This idea is framed from a socio-constructivist perspective (Slavin, 1985; Vygotsky, 1978). The social nature of cognition, as theorized by Vygotsky (1978), provides a basis for learning requiring social interaction.

Ultimately, social relationships contribute to group dynamics that are essential to developing a learning community (Rovai, 2002). Such a group-supported environment is conducive to effective learning (Foley, 1995; Knowles, 1990).

Research studies conducted in online learning environments have hypothesized social presence as a key component in the formation of community (Garrison et al., 2000; Gunawardena, 1995; Palloff \& Pratt, 1999; Wenger, 1998). Research has also hypothesized that a strong sense of community enhances learning support, commitment, collaboration, and learning satisfaction (Dede, 1996; Garrison et al., 2003; Hornik \& Johnson, 2003; Wellman, 1999).

This study, which examines students' perceptions of social presence in relation to students' satisfaction with learning and students' intent to enroll in a future online course, supports these hypotheses. The results from the quantitative and qualitative analyses found that when students perceived a moderate to high level of social presence, they were likely to feel more satisfied with their learning and indicated a desire to persist with enrollment in future online courses. On the other hand, when students perceived a low level of social presence, they were likely to feel dissatisfied with their learning and 
indicated a desire to acquire their course work through traditional means, if possible. Comments collected from the qualitative results also indicated that for students to learn, courses must be engaging, provoking them to interact, share ideas, and think critically. These findings suggest that positive perceptions of social presence may have an effect on group dynamics and the establishment of a sense of learning community in mediated communication.

Implications of the findings described in the previous sections from this study emphasize how the social interactions in online learning groups are strongly intertwined with learning interactions and the importance of considering skills that foster integration of social interactions or social presence within the educational transaction that takes place.

Implications of Social Presence in Online Learning

The environment in which learning takes place- whether online or in personinvolves a complex array of factors that influence learner satisfaction and achievement. Research on learning processes in face-to-face classrooms indicates that development of social climate is important in order to make students feel like insiders or a part of a community in the learning environment (Wegerif, 1998). It is claimed to be particularly important in technology-mediated learning situations (Harasim et al., 1995). The notion of forming a community of learners is an important issue in distance learning because it can affect student satisfaction, performance, and retention (Misanchuk et al., 2000; Neo, 2003).

In view of the literature presented that emphasizes the social dimension in construction of knowledge, this study explored students' perception of quality of learning 
and satisfaction with learning through the lens of social presence. Specifically, this study examined the relationships among these variables- social presence, satisfaction with learning, quality of learning, and intent to enroll in future online courses. In the context of an asynchronous computer-mediated learning environment and the theory of sociocultural learning, the findings of this study add empirical evidence to the distance education literature that social presence is a significant factor in positively affecting perceptions of learning quality, student satisfaction, and intent to enroll in future online courses.

Given these findings, the practical implications for course design and instruction will be presented. These implications will pertain to the pedagogical rationale and the role of instructors and students in supporting the creation of a social climate for online learning. It is not the technology that determines the quality of online learning but rather the pedagogical principles used to facilitate learning.

\section{Pedagogical Rationale}

Social behavior is an important factor in the development of learning processes. As other research studies have demonstrated along with these findings, it is essential that online courses be created in a way that enables students to interact, not only with the material, but with each other and instructors (Harasim, 1987; Hiltz, 1994; Irani, 1998; Roberson \& Klotz, 2002). As a result, new ways of understanding the material emerge as a result of the interaction that takes place with others in a learning community. Learning becomes a collaborative act among the participants, rather than the simple reception of information. A high level of social presence has been shown to enhance, foster, and increase interaction and participation $(\mathrm{Tu}, 2000)$. This research substantiates those claims 
that the perception of the amount and quality of social presence exert a strong influence on the level of satisfaction students derive from a course.

Adult learners benefit from the synergy and multiple perspectives provided in social learning situations (Candy, 1991; Garrison, 1997; Vygotsky, 1978) and, specifically, learning communities. A purposeful design of learning activities that are self-directed, reflect real-life experiences, and provide for collaboration and bonding can help support the goal of building learning communities online (Swan, 2002).

A collaborative approach in online learning is also particularly suited for corporations and training. Approximately $10-20 \%$ of all corporate training in the United States is presently being delivered via Web-based technologies resulting in well over one billion dollars of corporate training being spent on Web-based learning (Bucher, 2000; Kleingartner \& Jiang, 2001). While online learning has been widely used for training purposes, there is concern related to its effectiveness as student attrition from these courses is often cited as more than $50 \%$ (Bonk, 2004). It is presumable that learners' adoption or participation in online courses depends heavily on their perceived expectations of what they can achieve via this learning approach (Hodges, 2004). A study conducted by the American Society for Training and Development (2000) reveals that learners still prefer classroom-based training or abandon technology-based training after only a few sessions. Among the findings, factors that result in high dropout rates are poor incentives to learn due to lack of accountability for completing classes, and the inability of poorly designed courses that rely on static, superficial, and text-heavy learning content to hold a person's attention (Lake, 2000). 
Given that the ultimate goal of training is knowledge transfer to the job setting, it is important that attention be given to the instructional design that will motivate the adult learner to interact and collaborate with other professional peers, potentially drawing on a wider range of experience, to become skilled in problem-solving and project teamwork; skills that are in demand by today's business environment (Bonk, 2004; Mueller, 2003). Accordingly, online learning systems for corporate training, it would seem, should be structured to emphasize the social aspect of learning to engage the learners, foster interaction and collaboration, and improve satisfaction rather than simply employ a tool to provide repositories of information and track the learner's progress through that information. The result may improve participation, increase learning, and benefit the corporation.

Instructors should facilitate/moderate the learning activities in a purposeful and meaningful way in order to allow students to gain knowledge from each other and contribute to critical thinking (Kanuka \& Anderson, 1998). They should consider the character of the assignments in the course, provide practical guidelines or requirements for student participation in discussions, and moderate the discussions to sustain reciprocal interaction among learners and keep discussions on track. Online instructors need to provide instructional strategies that develop a community of learners and demand interaction and collaboration for the accomplishment of learning tasks.

\section{Role of Instructor and Student}

Instructors should be attentive to students' social needs and add interactions into learning activities to promote personal interaction among learners. As was noted from the students' comments in this study, it is crucial to enhance the social atmosphere by using 
supportive feedback, reinforcement, and by encouraging students to relate to each other during the learning activities. The research suggests that instructor presence in the online class is apparent to students when the instructor is visible to the student (Picciano, 2002). Because time and space separate the instructor from the students, more support and feedback from the instructor may be necessary than would be required in a face-to-face course. As a result, the support role of the instructor must be visible, consistent, and built into the overall structure of the course in the early stage of course design. This may require consistent activity in the form of feedback, assignment reminders, study guides, and other immediacy behaviors or response mechanisms.

Studies have indicated that immediacy, intimacy, and interaction are positive indicators of student learning outcomes and student satisfaction and they contribute to social presence (Christophel, 1990; Christophel \& Gorham, 1995; Gunawardena, 1995; Hackman \& Walker, 1990; Richardson \& Swan, 2003). Those communications cues that are attributed to social presence (immediacy, intimacy, and interaction) should be modeled by the instructor to encourage students to connect with other learners and sustain a learning community. Instructors should bridge the gap between the technology interface and the human factors involved in the learning process. Affectively, instructors need to acquire communication skills (praise, humor, and addressing students by name) that compensate for the lack of nonverbal cues in text-based online communication in order to create a sense of community with and among learners. Student perceptions of the social and human qualities of computer-mediated communication will depend on the social presence created by the instructor and the participants of the online community that 
is formed involving these communication cues (Gunawardena, 1995; Gunawardena \& Zittle, 1997).

Students' learning experiences are primarily shaped by the features of the traditional classroom. This perception was evident from those students in this study who primarily attributed their learning to that of the content only or to the interaction with their instructors and not with other learners. Students may avoid some interactions with other students if these interactions are viewed as being perfunctory and are not viewed as important components of the class. Consequently, students may need guidance to anticipate not only the changing role of the instructor but also their own role as a contributor in the process of construction of knowledge. Students should be encouraged to help each other and to refer to each other instead of looking to the instructor as the only resource for dialogue or help.

Learning experiences may benefit from being deliberately structured to promote interaction in support of social presence among students. Instructors should provide their expectations and guidelines revolving around appropriate engagement and participation to enhance students' ability and comfort in exchanging ideas with others. This may include initiating and focusing discussion topics, exchanging information, helping students connect ideas, and encouraging collaboration and open communication. This could create a feeling of trust and connection with other students and encourage students to go beyond minimum participation.

It was noted from the students' comments that the perception of social presence from their peers and their instructor minimized the psychological distance and influenced their perceived interaction and learning satisfaction from the online course activities. 
Furthermore, the results of this research demonstrated that students' perceived social presence and perceived satisfaction with learning influenced their plans to enroll in future online courses. These findings further substantiate the claims that the function of social presence can make the nature of online learning more interactive, engaging and intrinsically rewarding that could lead to increased persistence and course completion (Harasim et al., 1995; Misanchuk et al., 2000; Neo, 2003; Rourke et al., 2001). Therefore, it is important for online course designers and instructors to plan learning opportunities and strategies that would not only be most effective in meeting the learners' cognitive development, but also support the social climate.

\section{Limitations and Recommendations for Future Research}

A limitation of this study is the lack of randomization and control that characterizes experimental studies. As this was an exploratory study, generalization of the results beyond the present study are not valid because the participants were drawn from an "intact group" in one university who were enrolled in online courses and were able to provide the requested data via an online survey. An online instrument was appropriate for this study because the participants were available online and had, through their participation in an online class, demonstrated at least a basic level of skill with using online resources.

Previous studies on social presence have indicated a need for an investigation of a diverse sample of course content as many of these have focused primarily on graduate cohort participants enrolled in education or business courses (Gunawardena \& Zittle, 1997; Richardson, 2001; Shih, 2004; Tu, 2002). This study drew from online courses that represented both undergraduate and graduate level and involved nine disciplines. Due to 
the nature of variability in course types and number of online courses, this may be an important contribution to the empirical research in the field as most of the previous work has been done with fewer courses, limited disciplines, and smaller groups of students. However, the course content, course design, and the pedagogy used by instructors might have influenced the development of social presence. As such, these results may not be representative of other university settings and online course offerings.

The selection and size of the population used for this study may also limit the generalization of the findings. Future studies are warranted to investigate the variables of this study with different populations-- more diverse sample of course content, level of college credits, and online learning experience-- and in different learning environments, to further validate the findings of this study.

For example, future studies should be conducted with adult learners in different contexts outside of higher education such as in the workplace environment or in vocational training. Driven by the demands for learning opportunities to fulfill the needs of lifelong learning, the practitioners in the field of human resource development and training are striving for a more flexible and adaptive learning approach through online learning (Chen \& Chen, 2006). Over a billion dollars is being spent for online learning as a corporate training delivery method (Bucher, 2000). Yet, there is speculation as to whether online learning is appropriate for the workplace training due to reports of withdrawal rates as high as $80 \%$ (Bonk, 2004; Moshinskie, 2001). When considering the return on investment (ROI) for Web-based learning, an important element of measuring returns may be to capture the data on productivity, efficiency, and retention in completing online training. Further studies using these variables may help to serve a better 
understanding of what factors contribute to shaping learners' perceptions regarding whether online learning is an effective approach for corporate or workplace training.

The response rate to an Internet survey is another limitation. Electronic response rates tend to be lower when compared to mailed surveys. Low response rates may result in a biased estimate of the characteristics of the population (Bean \& Roszkowski, 1995). This study achieved about a $28 \%$ response rate from the participant pool. Although this response rate is consistent with previous research on Internet surveys, (Crawford et al., 2001; Simsek \& Veiga, 2001), additional studies using this research methodology should be done to further test the conceptual model proposed in this study. The demographic profile of the participants in this study was congruent with previous studies about distance learners as females comprised more than $60 \%$ of the sample and the majority were adults between the ages of 25-50 who selected an online course delivery mode primarily for the convenience (Hardy \& Boaz, 1997; Wolcott, 1996).

Another limitation is that the results from this research are reliant on students' self-reports and perceptions to the questions on the survey. Perceptions can be left open to interpretation and can involve attitudes, feelings, and beliefs that are subject to change over time. However, this does not discount the benefit that self-report surveys can provide as it is evident from reviewing other studies that there is value in measuring perceptions (Picciano, 2002). Ultimately, it is student perceptions of their learning that may be the catalyst for a student to continue their learning through Web-based courses.

Although this study used survey items to measure perceptions of social presence, satisfaction with learning, and intent to enroll that have been validated in other studies (Abraham, 1999; Gunawardena \& Zittle, 1997; Morton, 1993; Tu, 2002), follow-up 
studies should be conducted that use independent measures of social presence, the nature of interaction, and course satisfaction. These could include measures such as instructor ratings, content analyses of online communication, and examination of student interaction with course materials and other students through analysis of server logs.

Another limitation of this study is that it takes into consideration only the perceptions of the students who responded to the survey. The participants who chose to participate may represent a distinct population with characteristics that differ from the population as a whole. The viewpoints of the students who either did not respond or withdrew from the course before the end of the semester were not included in the data. Future studies should attempt to explore the viewpoints from those students who withdraw from online courses to more clearly understand if the absence of social presence or other factors may have played a stronger role in their decision.

Further research is needed that helps develop a better understanding of conditions or factors that may be both related and not related to online course completion. For example, $13 \%$ of the participants in this study reported they would enroll in another online course despite the lower perceptions of social presence and the poor quality of learning and satisfaction experienced within their current asynchronous online courses. The social interaction dimension of online learning communities may not be as important to those students who may be motivated or influenced by other attitudinal factors to succeed and enroll in future online courses. Research is needed to determine the extent that perception of social presence and/or motivation for learning support cognitive and affective learning objectives and student satisfaction, thereby promoting course completion and continued study. 
With respect to why students may be motivated to participate in Web-based learning, future studies should further explore the motives adult learners cite as the rationale for participating in online learning. Approximately $20 \%$ of the participants in this study cited either the enjoyment of interaction or curiosity as the rationale for taking the online course. Research has indicated that curiosity is an important motivator of adult learning because of its significant positive relationship to the information's perceived value to the learner (Rossing \& Long, 1981). Examining extrinsic and/or intrinsic motivation influences on participation in online learning may help to explain what factors direct and motivate learning and result in the outcome of course completion and retention in this learning context.

Given that students in this study experienced different levels of perceived social presence as well as satisfaction with learning, studies should be conducted to determine whether or not students are generally aware of the levels of learner-learner interaction they may experience and if this helps them to foster building relationships among learners and sustain a learning community in online courses.

Further research is needed to more clearly understand the effects of social presence in online courses. As this study could not establish a cause-effect relationship based on the statistical analyses between the variables that were examined, research needs to be done to determine if social presence is merely related to satisfaction and retention or if social presence in some way has the ability to cause these outcomes. The relationship between social presence and satisfaction and retention may be dependent on factors that are not clearly understood. These factors may be subject-centered such as student background with computer-mediated communication, student comfort with 
technology, locus of control or students who are self-directed learners, learning styles and study habits, student expectations of the course and the instructor, and motivation or attitudinal factors beyond that of desire for social presence and interaction with others.

There may also be structural factors intrinsic to the courses that affect interactions and the relationship between social presence and satisfaction beyond those examined in this study. These may include the nature of the subject matter; the quantity and quality of interaction with the instructor; the quantity and quality of interaction with other learners or the content; and access to adequate technical assistance along with the technology used to facilitate the interactions that take place in the course.

Only recently have studies begun to examine social presence as an important factor in the success of online learning (Picciano, 2000; Richardson \& Swan, 2003; Shih, 2004; Swan, 2001; Tu, 2000). In part, these studies inform us concerning the importance of the perception of social presence on interactions and the subjective measurement of learning outcomes in online courses. However, very few empirical studies have explained the relationship between social presence and persistence/retention in online learning.

This study extended the role of social presence in online courses. In addition to confirming the relationship between social presence and satisfaction with learning that has been previously established, this study also improved the understanding of the relationship by suggesting that social presence is also related to predicting persistence or intent to enroll again in a future online course. This finding needs to be carefully considered by course designers and instructors and additional studies should be conducted to test this premise further. The results could lead to a better understanding of 
what factors promote satisfaction with learning, course completion and continued study in an online environment or help to validate the Intent to Enroll model favored in this study that predicts a relationship between social presence and persistence with enrollment.

Summary

This study was designed to provide a better understanding of perceptions of social presence in relation to students' satisfaction with learning and retention in asynchronous learning environments. This chapter presented a discussion of the results of possible interaction or relationships between the variables explored in this study. These included perceived social presence and perceived satisfaction with learning; perceived social presence and perceived quality of learning in course activities; and perceived social presence, perceived satisfaction with learning, and intent to enroll in future online courses. The results suggested that social presence, a sense of connection with others despite not communicating face-to-face, was found to be a significant factor that contributed to students' perceptions of satisfaction and quality of learning, interaction, and in the development of learning communities within asynchronous online courses. Furthermore, the results demonstrated that a strong and positive relationship existed between social presence and persistence or intent to enroll in future online courses. A model related to this relationship was presented and discussed.

Moreover, the results from this study demonstrated that social presence plays an important role in fostering a worthwhile educational experience within a computermediated environment in terms of satisfaction and quality of learning with the course activities. In relation to perceptions of social presence and perceived satisfaction with 
learning, the results found that students' perceptions of the presence of others (instructors and peers) were closely related to the interactions they experienced in online course activities and, thus, contributed to their perceived satisfaction with learning. These findings imply that, if we agree that sustaining interactions in which collaborative construction of meaning among learners is an objective, then it is essential that online courses be designed with an emphasis on instructional methods and techniques that promote interaction, not only with the content, but also between instructor and learners and among learners themselves. This interaction, as it exists in the structures and practices of online courses, must be supported with activities and/or behaviors that add to perceptions of social presence to encourage students to connect with one another and sustain a learning community.

In summary, designers and instructors of online courses must address the requirements of effective educational environments that are of high quality, are effective, and that best meet the needs of the learner. The ability to express and share ideas among learners and with the instructor promotes collaboration and deepens the learning experience (Garrison, 1996). It is important to design online courses so they provide a satisfying and effective learning environment (Johnson, Aragon, Shaik, \& Palma-Rivas, 2000). The results of this study suggested that high levels of perceived social presence had a direct influence on both satisfaction with and perceived quality of learning in online courses. Furthermore, several viable design and instructional strategies were provided to address the variables in this study that could influence satisfaction with learning in online courses. 
By fostering an environment that will make the nature of online learning more interactive, appealing, engaging, and intrinsically rewarding through the creation of social presence, the levels of satisfaction and perceived quality of learning students derive from an online course, and ultimately, their motivation to persist with enrollment will be improved. The facilitation of student motivation and commitment will likely positively influence learning outcomes by improving students' involvement in the learning process, thus, contributing to more student interest in the content matter and enhancing collaboration during learning activities to construct knowledge and negotiate meaning through critical thinking.

It is hoped that the outcomes of this study, in examining the issues of social presence and its value in Web-based computer-mediated communication, can help to provide some valuable information and increase the understanding of how student needs, experiences, and perspectives can influence an optimal learning environment for online distance students. 


\section{REFERENCES}

Abraham, R. (1999). The impact of emotional dissonance on organizational commitment and intention to turnover. The Journal of Psychology, 133, 441-455.

Adams, S. (2004). The relationships among adult attachment, general self-disclosure, and perceived organizational trust. Unpublished doctoral dissertation, Virginia Polytechnic Institute and State University. Blacksburg, Virginia.

Agre, P.E. (1998). Designing genres for new media: Social, economic, and political contexts. In S.G. Jones (Ed.), Cybersociety 2.0: Revisiting computer mediated communication and community, 69-99. Thousand Oaks, CA: Sage Publications.

Althaus, S. (1997). Computer-mediated communication in the university classroom: An experiment with online discussions. Communication Education, 46, 158-174.

American Federation of Teachers (2001). A virtual revolution: Trends in the expansion of distance education. New York: American Federation of Teachers.

Anderson, J. (1979). Teacher immediacy as a predictor of teaching effectiveness. In D. Nimmo (Ed.), Communication Yearbook, 3, 543-559. New Brunswick, NJ: Transaction Books.

Anderson, S.E., \& Harris, J.B. (1997). Factors associated with amount of use and benefits obtained by users of a statewide educational teleconferencing network. Educational Technology Research and Development, 45, 19-50.

Anderson, T. (2002). Modes of interaction in distance education: Recent developments and research questions. In M. Moore and W. Anderson (Eds.), Handbook of Distance Education, 129-144. Mahwah, NJ: Lawrence Erlbaum Associates, Inc.

Aragon, S.R. (2003). Creating social presence in online environments. New Directions for Adult and Continuing Education, 100, 57-68.

Arbaugh, J.B. (2000). Virtual classroom characteristics and student satisfaction with Internet-based MBA courses. Journal of Management Education, 24, 32-54.

Argyle, M., \& Dean, J. (1965). Eye contact and distance affiliation. Sociometry, 28, 289-304. 
Astleitner, H. (2002). Teaching critical thinking online. Journal of Instructional Psychology, 2, 53-76.

Babbie. E. (1990). Survey research methods. Belmont, CA: Wadsworth.

Baird, J. (1977). The dynamics of organizational communication. New York: Harper and Row.

Barr, R.B., \& Tagg, J. (1995). From teaching to learning: A paradigm for undergraduate education. Change, 27, 12-25.

Bates, T. (1995). Technology, open learning and distance education. New York: Routledge.

Baym, N.K. (1998). The emergence in online community. In S.G. Jones (Ed.), Cybersociety 2.0: Revisiting computer-mediated communication and community, 35-68. Thousand Oaks, CA: Sage Publications.

Bean, A.G., \& Roszkowski, M.J. (1995). The long and short of it. Marketing Research, 7, 20-26.

Beaudin, B.P. (1999). Keeping online asynchronous discussions on topic. Journal of Asynchronous Networks, 3, 41-53.

Ben-Jacob M. (1998). Distance learning: An international perspective. Journal of Educational Technology Systems, 26, 209-213.

Bennett, G., \& Green, F.P. (2001). Student learning in the online environment: No significant difference? Quest, 1, 1-13.

Berge, Z. (1999). Interaction in post-secondary Web-based learning. Educational Technology, 39, 5-11.

Berge, Z. (2000). Components of the online classroom. In R.E. Weiss, D.S. Knowlton, \& B.W. Speck (Eds.), Principles of effective teaching in the online classroom. New Directions for Teaching and Learning, 84. San Francisco: Jossey-Bass.

Berge, Z., \& Collins, M. (1995). Computer-mediated communication and the online classroom. Creskill, NJ: Hampton Press.

Berge, Z., \& Collins, M. (2000). Perceptions of e-moderators about their roles and functions in moderating electronic mailing lists. Distance Education: An International Journal, 21, 81-100. 
Besser, H., \& Donahue, S. (1996). Introduction and overview: Perspective on distance independent education. Journal of the American Society for Information Science, $47,801-804$.

Bibeau, S. (2001). Social presence, isolation, and connectedness in online teaching and learning: From the literature to real life. Journal of Instruction Delivery Systems, $15,35-39$.

Biner, P.M., Birk, M.L., Huffman, M.L., \& Dean, R.S. (1995). Personality characteristics differentiating and predicting the achievement of televised-course students and traditional-course students. The American Journal of Distance Education, 9, 46-60.

Biocca, F., Harms, C., \& Burgoon, J. (2001). Criteria and scope conditions for a theory and measure of social presence. Presence 2001, $4^{\text {th }}$ Annual International Workshop, Philadelphia.

Blackboard 6.0 [Computer software]. (2003). Washington, D.C.: Blackboard, Inc.

Blocher, J.M. (1997). Self-regulation of strategies and motivation to enhance interaction and social presence in computer-mediated communication. Doctoral dissertation, Arizona State University. Dissertation Abstracts International, $03 \mathrm{~A}$.

Bonk, C.J. (2004). Online training in an online world. Education at a Distance, 16. [Online]. Available from http://www.usdla.org/html/journal/MAR02_Issue/article02.html

Bradley, N. (1999). Sampling for Internet surveys: An examination of respondent selection for Internet research. Journal of the Market Research Society, 41, 387395.

Brady, E. \& Bedient, D. (2003). The effects of teacher presence on student performance and attitudes. $5^{\text {th }}$ Annual WebCT User Conference Proceedings.

Brandon, D.P., \& Hollinghead, A.B. (1999). Collaborative learning and computersupported groups. Communication Education, 48, 109-126.

Brown, J.D. (2000). What issues affect Likert-scale questionnaire formats? Journal of Language and Testing, 4, 18-21.

Bruner, J. (1990). Acts of meaning. Cambridge, MA: Harvard University Press.

Bucher, J. (2000). Revolutionizing corporate training and strategy. Fortune Special Sections (May). [Online]. Available from http://www.fortune.com/fortune/sections/online learn/onlinelearn.htm 
Bullen, M. (1998). Participation and critical thinking in online university distance education. Journal of Distance Education, 9, 19-43.

Burge, E.J. (1994). Learning in computer conferenced contexts: The learner's perspective. Journal of Distance Education, 13, 51-65.

Cahoon, B. (1998). Adult learning and the Internet: Themes and things to come. In B. Cahoon (Ed.), Adult learning and the Internet, 63-69. San Francisco, CA: JosseyBass Publishers.

Cammann, C., Fichman, M., Jenkins, D., \& Klesh, J. (1979). The Michigan Organizational Assessment Questionnaire. Unpublished manuscript, University of Michigan, Ann Arbor.

Candy, P. (1991). Self-direction for lifelong learning. San Francisco, CA: Jossey-Bass.

Carr, A. (2000). As distance education comes of age, the challenge is keeping the students. The Chronicle of Higher Education, 46, A39-A42.

Carswell, L. (2000). Distance education via the Internet: The student experience. British Journal of Educational Technology, 31, 29-46.

Charp, S. (1998). Distance learning (Editorial). T.H.E. Journal, 26, 4.

Chen, T.L. \& Chen, T.J. (2006). Exploring learner perceptions of e-learning effectiveness in the workplace learning context based on diffusion of innovations (DOI) model. Paper presented at the American Human Resource Development Conference. Columbus, $\mathrm{OH}$.

Chickering, A.W., \& Gamson, Z.R. (1991). Seven principles for good practice in undergraduate education. In A.W. Chickering \& Z.E. Gamson (Eds), Applying the seven principles for good practice in undergraduate education, 63-69. San Francisco, CA: Jossey-Bass.

Christophel, D.M., \& Gorham, J. (1995). A test-retest analysis of student motivation, teacher immediacy, and perceived sources of motivation and demotivation in college classes. Communication Education, 44, 292-306.

Clarke, R. (1989). Going the distance. Black Enterprise, 29, 13-118.

Cohen, J. (1988). Statistical power analysis for the behavioral sciences, (2 ${ }^{\text {nd }}$ ed.). New Jersey: Lawrence Erlbaum.

Cohen, \& Cohen (1983). Applied multiple regression/correlation analysis for the behavioral sciences, $\left(2^{\text {nd }}\right.$ ed.). Hillsdale, NJ: Erlbaum Associates. 
Collins, M. (2000). Comparing Web, correspondence and lecture versions of a secondyear non-major biology course. British Journal of Educational Technology, 31, $21-27$.

Collins, M., \& Berge, Z. (1996). Facilitating interaction in computer-mediated online courses. [Online ]. Available from http://www.emoderatots.com/moderators/flcc.html.

Connick, G.P. (1999). The distance learner's guide. Upper Saddle River, NJ: Prentice Hall.

Connolly, T., Jessup, L.M., \& Valacich, J.S. (1990). Effects of anonymity and evaluative tone or idea generation in computer-mediated groups. Management Science, 36, $97-120$.

Cook, C., Heath, F., \& Thompson, R. (2000). A meta-analysis of response rates in Webor Internet-based surveys. Educational Psychological Measurement, 60, 821-836.

Cookson, P. (1990). Persistence in distance education. In M.G. Moore (Ed.), Contemporary issues in American distance education. New York: Pergamon Press.

Couper, M.P. (2002). New technologies and survey data collection: Challenges and opportunities. International Conference on Improving Surveys, Copenhagen. [Online]. Available from http://www.icis.dk/papers.html.

Cravner, P.A., \& Michael, W.B. (1998). Students' use of adjunctive CMC. Paper presented at the $5^{\text {th }}$ Annual Distance Education Conference, Texas A \& M University.

Crawford, S.D., Couper, M.P., \& Lamias, M.J. (2001). Web surveys: Perceptions of burden. Social Science Computer Review, 19, 146-162.

Crebbin, W. (1999). How does learning happen? In G. Crosling, T. Moore, \& S. Vance (Eds.), Referred Proceedings of the National Language and Academic Skills Conference, 13-19. Churchill, Vic: Celts, Monash University.

Creswell, J.W. (1994). Research design: Qualitative and quantitative approaches. Thousand Oaks, CA: Sage.

Cutler, R. (1995). Distributed presence and community in cyberspace. Interpersonal computing and technology: An electronic journal for the $21^{\text {st }}$ century, 31, 323340 . 
Danchak, M.M., Walther, J.B., \& Swan, K. (2001). Presence in mediated instruction: Bandwidth, behavior, and expectancy violations, $7^{\text {th }}$ Sloan-C International Conference on Online Learning. Orlando, FL.

Daniel, J.S., \& Marquis, C. (1979). Interaction and independence: Getting the mixture right. Teaching at a Distance, 14, 29-43.

Daugherty, M., \& Funke, B.L. (1998). University faculty and student perceptions of Web-based instruction. Journal of Distance Education, 13, 21-39.

Dede, C. (1995). The transformation of distance education to distributed learning. International Technology Research Online. [Online]. Available from http://www.gsu.edu/wwwitr/docs/distlearn/index.html.

Dede, C. (1996). The evolution of distance education: Emerging technologies and distributed learning. American Journal of Distance Education, 10, 4-36.

Dillon, C. (1989). Faculty rewards and instructional telecommunications: A view from the telecourse faculty. American Journal of Distance Education, 3, 35-43.

Dillman, D.A. (1978). Mail and telephone surveys: The total design method. New York: John Wiley \& Sons.

Dillman, D.A. (2000). Mail and internet surveys: The tailored design method. (2 ${ }^{\text {nd }}$ ed.). New York: John Wiley \& Sons.

Dillman, D.A., \& Bowker, D.K. (2001). The web questionnaire challenge to survey methodologists. [Online]. Available from http://www.survey.sesrc.wsu.edu/dillman/zuma paper dillman bowker.pdf

Dillman, D.A., Tortora, R.D., \& Bowker, D.K. (1999). Principles for constructing web surveys. [Online]. Available from http://survey.sesrc.wsu.edu/dillman/papers/websurveyppr.pdf

Dillon, C.L., \& Walsh, S.M. (1992). Faculty: The neglected resource in distance education. In L. Foster, B. Bower, and L. Watson, (Eds.), Teaching and learning in higher education, ASHE Reader Series.

Dormant, D. (1999). Implementing human performance technology in organizations. In H. Stolovitch \& E. Keeps (Eds.), Handbook of human performance technology, ( $2^{\text {nd }}$ ed.). San Francisco, CA: Jossey-Bass/Pfeiffer.

Dziuban, C., \& Moskal, P. (2001). Emerging research issues in distributed learning. Paper presented at $7^{\text {th }}$ Sloan-C International Conference on Asynchronous Learning Networks. Orlando, FL. 
Eastmond, D.V. (1995). Alone but together: Adult distance study through computer conferencing. Cresskill, NJ: Hampton Press.

Edelson, P.J. (1998). The organization of courses via the Internet, academic aspects, interaction, evaluation, and accreditation. Paper presented at the National Autonomous University of Mexico. (Mexico City, Mexico, February 17, 1998).

El-Tigi, M., \& Branch, R.M. (1997). Designing for interaction, learner control, and feedback during Web-based learning. Educational Technology, 3, 23-29.

Evard, R., Churchill, E, \& Bly, S. (2001). Waterfall Glen: Social virtual reality at work. In E.F. Churchill, D.N. Snowdon, \& A.J. Munro (Eds.), Collaborative virtual environments: Digital places and spaces for interaction. London: SpringerVerlag.

Fabro, K.R., \& Garrison, D.R. (1998). Computer conferencing and higher-order learning. Indian Journal of Open Learning, 7, 41-53.

Fast, M. (1995). Interaction in technology: Mediated, multisite, foreign language instruction. Paper presented at the Annual Meeting of the American Educational Research Association. San Francisco, CA.

Fink, A. (1995). How to ask survey questions. Thousand Oaks, CA: Sage.

Fjortoft, N.F. (1996). Persistence in a distance learning program: A case in pharmaceutical education. The American Journal of Distance Education, 10, 4959 .

Flanders, N. (1970). Analyzing teacher behavior. Reading, MA: Addison-Wesley.

Foley, G. (1995). Teaching adults. In G. Foley (Ed.), Understanding Adult Education and Training, 31-53. Sydney: Allen \& Unwin.

Forsman, G., \& Varedian, M. (2002). Mail and web surveys: A cost and response rate comparison in a study of student housing conditions. International Conference on Improving Surveys, Copenhagen, Denmark, [Online]. Available from http://www.icis.dk/papers.html.

Fowler, F.J. (1984). Survey research methods. Beverly Hills, CA: Sage.

Fowler, F.J. (1993). Survey research methods, ( $3^{\text {rd }}$ ed.). Newberry Park, CA: Sage.

Fulford, C.P., \& Zhang, S. (1993). Perception of interaction: The critical predictor in distance education. The American Journal of Distance Education, 7, 8-21. 
Fyfe, S. (2000). Collaborative learning at a distance: The human biology experience. Paper presented at the $9^{\text {th }}$ Annual Teaching Learning Forum, February 2-4, 2000, Perth Australia. [Online]. Available from http://www.lsn.curtin.edu.au/tlf/tlf/2000/fyfes.html.

Gall, M.D., Borg, W.R., \& Gall, J.P. (1996). Educational research: An introduction, (6 ${ }^{\text {th }}$ ed.). White Plains, NY: Longman.

Garland, R. (1991). The mid-point on a rating scale: Is it desirable? Marketing Bulletin, 2, 66-70.

Garrison, D.R. (1989). Understanding distance education: A framework for the future. London: Routledge.

Garrison, D.R. (1990). An analysis and evaluation of audio teleconferencing to facilitate education at a distance. The American Journal of Distance Education, 4, 16-23.

Garrison, D.R. (1993). Quality and access in distance education: Theoretical considerations. In D. Keegan (Ed.), Theoretical principles of distance education, 9-21. New York: Routledge.

Garrison, D.R. (1995). Constructivism and the role of the self-instructional course materials: A reply. Distance Education, 16, 136-140.

Garrison, D.R. (1996). Computer conferencing: The post-industrial age of distance education. Manuscript submitted for publication.

Garrison, D.R. (1997). Self-directed learning: Toward a comprehensive model. Adult Education Quarterly, 41, 125-149.

Garrison, D.R., Anderson, T, \& Archer, W. (2000). Critical inquiry in a text-based environment: computer conferencing in higher education. The Internet and Higher Education, 2, 1-19.

Garrison, Anderson, T., \& Archer, W. (2003). A theory of critical inquiry in online distance education. In M. Moore \& W. Anderson (Eds.), Handbook of Distance Education, 113-127. Mahwah, NJ: Lawrence Erlbaum Assoc., Inc.

Geelan, D.R., \& Taylor, P.C. (2000). Promoting open and critical discourse in online learning. In C. Beasley (Ed.), Released Proceedings of the Third Biennial Communication Skills in University Education Conference. Murdoch, WA: Teaching and Learning Centre, Murdoch University.

Gibson, C.C. (1996). Toward an understanding of academic self-concept in distance education. The American Journal of Distance Education, 10, 23-36. 
Gilbert, L., \& Moore, D.R. (1998). Building interactivity into web courses: Tools for social and instructional interaction. Educational Technology, 38, 29-35.

Gokhale, A. (1995). Collaborative learning enhances critical thinking. Journal of Technology Education, 7, 1-2.

Gorham, J. (1988). The relationship between verbal teacher immediacy behaviors and student learning. Communication Education, 37, 40-53.

Gredler, M.E. (1997). Learning and instruction: Theory into practice. Upper Saddle River, NJ: Prentice Hall.

Gunawardena, C.N. (1992). Changing faculty roles for audiographics and online teaching. American Journal of Distance Education, 6, 58-71.

Gunawardena, C.N. (1995). Social presence theory and implications for interaction and collaborative learning in computer conferences. International Journal of Educational Telecommunications, 1, 147-166.

Gunawardena, C.N., Lowe, C.A, \& Anderson T. (1997). Analysis of global online debate and the development of an interaction analysis model for examining social construction of knowledge in computer conferencing. Journal of Educational Computing Research, 17, 397-431.

Gunawardena, C.N., \& Zittle, F.J. (1997). Social presence as a predictor of satisfaction within a computer-mediated conferencing environment. American Journal of Distance Education, 11, 8-26.

Hackman, M.Z., \& Walker, K.B. (1990). Instructional communication in the televised classroom: The effects of system design and teacher immediacy on student learning and satisfaction. Communication Education, 39, 196-206.

Hannifin, M.J., \& Land, S.M. (1997). The foundations and assumptions of technologyenhanced student-centered learning environments. Instructional Science, 25, 167202.

Harasim, L.M. (1987). Teaching and learning online: Issues in computer-mediated graduate courses. Canadian Journal of Educational Communication, 16, 117135.

Harasim, L.M. (1989). Online education: A new domain. In R. Mason and A. Kaye (Eds.), Mindweave: Communication, computers and distance education, 50-62. Oxford: Pergamon Press. 
Harasim, L.M. (1990). Online education: An environment for collaboration and intellectual amplification. In L.M. Harasim (Ed.), Online education: Perspectives on a new environment, 39-64. New York: Praeger.

Harasim, L.M. (1993). Global networks: Computers and international communication. Cambridge, MA: MIT Press.

Harasim, L.M. (1995). Learning networks: A field guide to teaching and learning online. Cambridge, MA: MIT Press.

Harasim, L.M. (1996). Online education: In T.M. Harrison \& T. Stephen (Eds.), Computer networking and scholarly communication in the twenty-first-century university, 203-214. Albany, NY: State University of New York Press.

Harasim, L.M., Hiltz, S.R., Teles, K., \& Turoff, M. (1995). Learning networks: A field guide to teaching and learning online. Cambridge, MA: MIT Press.

Harasim, L.M., \& Yung, B. (1993). Teaching and learning on the Internet. Burnaby, BC: Department of Communications, Simon Frasier University.

Hardy, D.W., \& Boaz, M.H. (1997). Learner development: Beyond the technology. In T. Cyrs (Ed.), New Directions for Teaching and Learning, 71, 41-48. San Francisco, CA: Jossey-Bass.

Harel, I., \& Papert, S. (Eds.), (1991). Constructionism. Norwood, NJ: Ablex.

Hart, G. (2003). Power literacy and motivation. Florida Department of Education. [Online]. Available from http://www.gseweb.harvard.edu/ ncsall/fob/1998/hart.html.

Heath, R.L., \& Bryant, J. (1992). Human communication theory and research: Concepts, contexts, and challenges. In J. Bryant (Ed.), Communication textbook series: General communication theory and methodology. Hillsdale, NJ: Erlbaum.

Henri, F. (1991). Computer conferencing and content analysis. In A. Kaye (Ed.), Collaborative learning through computer conferencing. Heidelberg, FRG: Springer-Verlag.

Henri, F. (1995). Distance learning and computer-mediated communication: Interactive, quasi-interactive, or monologue? In C. O'Malley (Ed.), Computer supported collaborative learning, 15-161. London: Springer-Verlag.

Herring, S.C. (2000). Gender differences in CMC: Findings and implications. The CPSR Newsletter, 18, 3-11. 
Hiemstra, R. (1991). Aspects of effective learning environments. In R. Hiemstra (Ed.), Creating environments for effective adult learning. New Directions for Adult and Continuing Education, 50, 5-11.

Hill, J. (1997). Distance learning environments via the World Wide Web. In B. Kahn (Ed.), Web-based instruction, 75-80. Englewood Cliffs, NJ: Educational Technology Publications.

Hill, J. (1999). Learning about distance education at a distance: Rewards and challenges. Paper presented at American Educational Research Association. Montreal, Canada.

Hillman, D. (1999). A new method for analyzing patterns of interaction. American Journal of Distance Education, 13, 37-47.

Hillman, D.C., Willis, D.J., \& Gunawardena, C.N. (1994). Learner-interface interaction in distance education: An extension of contemporary models and strategies for practitioners. American Journal of Distance Education, 8, 30-42.

Hiltz, S.R. (1986). The "virtual classroom": Using computer-mediated communication for university teaching. Journal of Communication, 36, 95-104.

Hiltz, S.R. (1990). Evaluating the virtual classroom. In L.M. Harasim (Ed.), Online education: Perspectives on a new environment. 133-183. New York: Praeger.

Hiltz, S.R. (1994; 1998). The virtual classroom: Learning without limits via computer networks. Norwood, NJ: Ablex Publishing.

Hiltz, S.R. (1997). Impacts of college-level courses via asynchronous learning networks: some preliminary results. Journal of Asynchronous Learning Networks, 1. [Online]. Available from http://www.aln.org/alnweb/journal/issue2/hiltz.html.

Hiltz, S.R., Coppola, N., Rotter, N., Turoff, M., \& Benbunan-Fich, R. (2001). Measuring the importance of collaborative learning for the effectiveness of ALN: A multimeasure, multi-method approach. Journal of Asynchronous Learning Networks, 4. [Online]. Available from http://www.aln.org/alnweb/journal/Vol14 issue2/le/hiltz/le hiltz.html.

Hodges, C.B. (2004). Designing to motivate: Motivational techniques to incorporate in elearning experiences. The Journal of Interactive Online Learning, 2. [Online]. Available from http://www.nclor.org

Holmberg, B. (1983). Guided didactic conversation in distance education. In D. Sewart, S. Keegan, B. Holmberg (Eds.), Distance education: International perspectives. London: Routledge. 
Hong, K., Lai, K., \& Holton, D. (2003). Students' satisfaction and perceived learning with a Web-based course. Educational Technology and Society, 6, 1-14.

Hornik, S., \& Johnson, R. (2003). Hello, hello, is there anybody in there? The role of perceived social presence in technology-mediated learning environments. (Working paper).

Huang, A.H. (1997). Challenges and opportunities of online education. Journal of Educational Technology Systems, 25, 229-247.

Huff, M.T. (1998). A comparison of critical thinking in an interactive television social work course. Doctoral dissertation, University of South Carolina, Columbia. Dissertation Abstracts International. Ann Arbor, MI: UMI Press.

Ingram, D., \& Sweeney, J.C. (2000). Cultural differences in student perceptions of communicating and learning through traditional and web-based tutorials. In C. Beasley (Ed.), Refereed Proceedings of the Third Biennial Communication Skills in University Education Conference, 73-80. Murdoch, WA: Teaching and Learning Centre, Murdoch University.

Imel, S. (1988). Guidelines for working with adult learners. ERIC Digest No. 77. (ERIC Document Reproduction Service No. ED299458).

Irani, T. (1998). Communication potential, information richness and attitude: A study of computer-mediated communication in the ALN classroom. ALN Magazine, 2, 726.

Isaac, S., \& Michael, W.B. (1995). Handbook in research and evaluation: A study of principles, methods, and strategies useful in the planning, design, and evaluation of studies in education and the behavioral sciences. San Diego, CA: EdITS.

James, D., \& Drakich, J. (1993). Understanding gender differences in amount of talk: A critical review of research. In D. Tannen (Ed.), Gender and conversational interaction. New York: Oxford University Press.

Jiang, M. (1998). Distance learning in a Web-based environment: An analysis of factors influencing students' perceptions of online learning. Doctoral dissertation, University of Albany, State University of New York. Dissertation Abstracts International, UMI 9913679. Ann Arbor, MI: UMI Press.

Jiang, M., \& Ting, E. (2000). A study of factors influencing students' perceived learning in a Web-based course environment. International Journal of Telecommunication, 6, 317-338. 
Johnson, S.D., Aragon, S.R., Shaik, N., \& Palma-Rivas, N. (2000). Comparative analysis of learner satisfaction and learning outcomes in online and face-to-face learning environments. Journal of Interactive Learning Research, 11, 29-49.

Johnson, M.M., \& Huff, M.T. (2000). Students' use of computer-mediated communication in a distance education course. Research on Social Work Practice, 10, 519-532.

Johnson, D.W., \& Johnson, R.T. (1999). Learning together and alone: Cooperative competitive and individualistic learning $\left(5^{\text {th }} \mathrm{ed}\right)$. Needham Heights, MA: Allyn and Bacon.

Jonassen, D.H. (2000). Technology and constructivism. In M. Moore \& N. Shin (Eds.), Speaking personally about distance education: Foundations of contemporary practice. University Park, PA: Pennsylvania State University.

Jonassen, D.H., Davidson, M., Collins, M., Campbell, J., \& Haag, B. (1995). Constructivism and computer-mediated communication in distance education. The American Journal of Distance Education, 9, 7-26.

Jones, S.G. (1998). Cybersociety 2.0: Revisiting computer-mediated communication and community. Thousand Oaks, CA: Sage.

Jung, I.S., \& Leem, J.H. (1999). Training manual for the design of Web-based instruction. Korea National Open University.

Jung, I.S., Lim, C.I., Choi, S.H., \& Leem, J.H. (1998). Development of teaching-learning models for WBI for lifelong education. Korea Foundation for Research, policy paper.

Kanuka, H., \& Anderson, T. (1998). Online social interchange, discord, and knowledge construction. Journal of Distance Education, 13, 57-74.

Kaye, T. (1987). Computer conferencing and electronic mail. ERIC Digest. (ERIC Document Reproduction Service No. ED329209).

Kaye, A.R. (Ed.), (1992). Collaborative learning through computer-conferencing: The Najaden Papers. New York: Springer-Verlag.

Kearney, P., Plax, T.G., \& Wendt-Wasco, N.J. (1986). Teacher immediacy for affective learning in divergent college classes. Communication Quarterly, 33, 61-74.

Kearsley, G. (1995). The nature and value of interaction in distance learning. Paper presented at the International Research Conference in Distance Education: A Research Agenda. 
Kearsley, G. (2000). Online education: Learning and teaching in cyberspace. Belmont, CA: Wadsworth.

Kearsley, G., \& Shneiderman, B. (1998). Emergent patterns of teaching/learning in electronic classrooms. Educational Technology Research and Development, 46, $23-42$.

Keegan, D. (1988). On defining distance education. Distance Education, 1, 13-36.

Keegan, D. (1990). Foundations of Distance Education, (2 ${ }^{\text {nd }}$ ed.). New York: Routledge.

Kelley, D.H., \& Gorham, J. (1988). Effects of immediacy on recall of information. Communication Education, 37, 198-207.

Kember, D. (1989). A longitudinal process model of dropout from distance education. Journal of Higher Education, 60, 278-301.

Kerka, S. (1996). Distance learning, the Internet, and the World Wide Web. ERIC Digest. (ERIC Document Reproduction Service No. ED 395-214).

Kiesler, S., \& Sproull, L.S. (1986). Response effects in the electronic survey. Public Opinion Quarterly, 50, 402-413.

Kleingartner, A. \& Jiang, R. (2001). Performance and web-based learning. Paper posted at e-Scholarship Repository, University of California. [Online]. Available from http://www.repositories.cdlib.org/ile/scl2001/Section 11/html

Knowles, M. (1975). Self-directed learning. New York: Association Press.

Knowles, M. (1980). The modern practice of adult education: From pedagogy to andragogy. ( $\left.2^{\text {nd }} \mathrm{ed}.\right)$. New York: Cambridge Books.

Knowles, M.S. (1990). The adult learner: A neglected species $\left(4^{\text {th }}\right.$ ed.). Houston, TX: Gulf

Knowles, M.S. (1996). Adult learning. Chapter 12 in R.L. Craig (Ed.), The ASTD training and development handbook ( $4^{\text {th }}$ ed.). New York: McGraw-Hill.

Kraut, A.I. (1996). Introduction and overview of organizational surveys. In A.I. Kraut (Ed.), Organizational surveys: Tools for assessment and change, 101-110. San Francisco, CA: Jossey-Bass.

Kulik, C., \& Kulik, J. (1991). Effectiveness of computer-based instruction: An updated analysis. Computers and Human Behavior, 7, 75-94. 
Laurillard, D. (1993). Rethinking university teaching: A framework for the effective use of educational technology. London: Routledge

Lauzon, A.C. (1992). Integrating computer-based instruction with computer conferencing: An evaluation of a model for designing online education. American Journal of Distance Education, 6, 32-46.

Lea, M. (Ed.), (1992). Contexts of computer-mediated communication. New York: Harvester.

Leh, A.S. (2001). Computer-mediated communication and social presence in a distance learning environment. International Journal of Telecommunications, 7, 109-128.

Lesser, V.M., \& Newton, L. (2002). Comparison of response rates and quality of response in a survey conducted by mail, email, and Web. $57^{\text {th }}$ Annual Conference of the American Association for Public Opinion Research, St. Petersburg, Florida. Available from Department of Statistics, Oregon State University, Corvallis, Oregon, 97331.

Lockhart, D.C., \& Russo, J.R. (1996). Mail and telephone surveys in marketing research: A perspective from the field. In P. Bagozzi (Ed.), Principles of marketing research, 17-161. Cambridge, UK: Blackwell.

Lombard, M., \& Ditton, T. (1997). At the heart of it all: The concept of presence. Journal of Computer-Mediated Communications, 3, 301-321.

Luk, S.C. (1998). The relationship between cognitive style and academic achievement. British Journal of Educational Technology, 29, 137-147.

Lynch, M. M. (2001). Effective student preparation for online learning. The Technology Source. [Online]. Available from http://www.ts.mivu.org/default.asp?show=article\&id

Malikowski, S. (1997). Interacting in history's largest library: Web-based conferencing tools. In B.H. Kahn (Ed.), Web-based instruction, 283. Englewood cliffs, NJ: Educational Technology Publications.

Manfreda, K.L., Batagelj, Z., \& Vehovar, V. (2002). Design of Web survey questionnaires: Three basic experiments. Journal of Computer-Mediated Communications, 7. [Online]. Available from http://www.ascusc.org/jemc/vol7/issie3/vehovar.html.

Mangione, T.W. (1998). Mail surveys. Chapter 14 in Bickman, L., \& Rog, D.J. (Eds.), Handbook of applied social research methods. Thousand Oaks, CA: Sage. 
Mason, R., \& Kaye, A.R. (1990). Towards a new paradigm for distance education. In L.M. Harasim (Ed.), Online education: Perspectives on a new environment, 279288. New York: Praeger.

Matell, M.S., \& Jacoby, J. (1972). Is there an optimal number of alternatives for Likert scale items? Effects of testing time and scale properties. Journal of Applied Psychology, 56, 506-509.

McDonald, J., \& Gibson, C.C. (1998). Interpersonal dynamics and group development in computer conferencing. American Journal of Distance Education, 12, 7-25.

McInnerney, J.M., \& Roberts, T.S. (2004). Online learning: Social interaction and the creation of a sense of community. Educational Technology and Society, 7, 73-81.

McIsaac, M.S., Blocher, J.M., Mahes, V., \& Vrasidas (1999). Student and teacher perceptions of interaction in online computer-mediated communication. Educational Media International, 36, 121-131.

McIsaac, M.S., \& Gunawardena, C.N. (1996). Distance education. In D. Jonassen (Ed.), Handbook for educational communications and technology, 403-437. New York: Scholastic Press.

McLoughlin, C., \& Luca, J. (2002). Enhancing the quality of the student experience online: Revisiting the imperative of learning as socially based. HERDSA, 442448.

McMahon, T.A. (1997). From isolation to interaction? Network-based professional development and teacher professional communication. Paper presented the annual meeting of the American Educational Research Association, Chicago, IL. (ERIC Document Reproduction Service No. ED 408 257).

Mehrabian, A. (1967). Attitudes inferred from nonimmediacy of verbal communication. Journal of Verbal Learning and Verbal Behavior, 6, 294-295.

Mehrabian, A. (1969). Some referents and measures of nonverbal behavior. Behavior Research Methods and Instrumentation, 1, 205-207.

Mehrotra, C., Hollister, C., \& McGahey, L. (2001). Distance learning: Principles for effective design, delivery, and evaluation. Thousand Oaks, CA: Sage.

Mehta, R., \& Sivadas, E. (1995). Comparing response rates and response content in mail versus electronic surveys. Journal of the Market Research Society, 4, 429-440.

Merriam, S.B., \& Brockett, R.G. (1997). The profession and practice of adult education: An introduction. New Directions for Adult and Continuing Education, 50, 5-11. San Francisco, CA: Jossey-Bass. 
Merriam, S.B., \& Caffarella, R. (1999). Learning in adulthood: A comprehensive guide. San Francisco, CA: Jossey-Bass.

Merrill, M.D. (2002). First principles of instruction. Educational Technology Research and Development, 50, 43-59.

Misanchuk, M., Anderson, T., Caner, J., Eddy, P., \& Smith, C. (2000). Building community in an online learning environment: Communication, cooperation, and collaboration, 1-17. [Online]. Available from http://www.mtsu.edu/ itconf/proceed01/19.html.

Moore, M. (1989). Three types of interaction. American Journal of Distance Education, $3,1-6$.

Moore, M. (1993). Theory of transactional distance. In D. Keegan (Ed.), Theoretical Principles of Distance Education, 22-38. New York: Routledge.

Moore, M., \& Kearsley, G. (1996). Distance education: A systems view. New York: Wadsworth.

Moore, A., Masterson, J.T., Christophel, D.M., \& Shea, K.A. (1996). College teacher immediacy and student ratings of instruction. Communication Education, 45, 2939.

Morton, S. (1993). Socialization-related learning, job satisfaction, and commitment for new employees in a federal agency. Unpublished doctoral dissertation, Virginia Polytechnic Institute and State University.

Moshinskie, J. (2001). How to keep e-learners from escaping. Performance Improvement, 40, 28-35.

Mueller, B. (2003). Online education in the corporate context. Chief Learning Officer: Solutions for Enterprise Productivity. (October). [Online]. Available from http://www.clomedia.com/content/templates/clo feature.asp?articleid=271

Muirhead, B. (2001). Enhancing social interaction in computer-mediated distance education. Education at a Distance, 15, Article 2. [Online]. Available from http://www.usdla.org/html/journal/APR01 Issue/article02 html.

Mummery, J. (2002). Facilitating critical thinking in an online environment. HERDSA, 465-471.

Murphy, P.R., Daley, J., \& Dalenberg, D.R. (1991). Exploring the effects of postcard pre-notification on industrial firms' response to mail surveys. Journal of the Market Research Society, 33, 335-345. 
Naidu, S. (1997). Collaborative reflective practice: Instructional design architecture for the Internet. Distance Education, 18, 257-283.

National Center for Education Statistics (1999). Distance education at post-secondary institutions, 1997-1998: Statistical analyses report. (NCES-2000-013).

Neo, K. (2003). Using multimedia in a constructivist learning environment in the Malaysian classroom. Australian Journal of Educational Technology, 19, 293310.

Newberry, B. (2001). Raising student social presence in online classes. In WebNet 2001. Proceedings of the World conference on the WWW and Internet. AACE, 2001. Norfolk, VA.

Norman, A. (1998). The design of everyday things. London: MIT Press.

Northrup, P. (2001). A framework for designing interactivity in Web-based instruction. Educational Technology, 4, 31-39.

Norton, R.W. (1986). Communicator style in teaching: Giving good form to content. In J.M. Civikly (Ed.), Communicating in college classrooms, 33-40. San Francisco, CA: Jossey-Bass.

Oliver, R. (1999). Exploring strategies for online teaching and learning. Distance Education, 20, 240-254.

Osgood, C.E., Suci, G.J., \& Tannenbaum, P.H. (1957). The measurement of meaning. Urbana, IL: University of Illinois Press.

Pagano, R.R. (1998). Understanding statistics in the behavioral sciences $\left(5^{\text {th }} \mathrm{ed}\right.$.). Pacific Grove, CA: Brooks/Cole.

Palloff, R.M., \& Pratt, K. (1999). Building learning communities in cyberspace: Effective strategies for the online classroom. San Francisco, CA: Jossey-Bass.

Palloff, R.M., \& Pratt, K. (2001). Lessons from the cyberspace classroom. San Francisco, CA: Jossey-Bass.

Papert, S. (1993). The children's machine: Rethinking school in the age of the computer. New York: Basic Books.

Parker, A. (1996). Distance education attrition. International Journal of Educational Telecommunications, 1, 389-406. 
Parker, A. (1999). Interaction in distance education: The critical conversation. Educational Technology Review, 12, 15-17.

Paulsen, M.F. (1995). The online report on pedagogical techniques for computermediated communication. [Online]. Available from http://www.hs.nki.no/ morten/cmcped.html.

Penna-Shaff, J., Martin, W., \& Gay, G. (2001). An epistemological framework for analyzing student interactions in computer-mediated communication environments. Journal of Interactive Learning Research, 12, 41-68.

Pearse, E.M., Burton, P.I., Kovner, E.S., Lears, M.E., \& Sen, R.J. (1992). Predicting computer-mediated communication in a college class. Communication Research Reports, 9, 161-170.

Phillips, J., Phillips, P., \& Zuniga, L. (2000). Evaluating the effectiveness and the return on investment of e-learning. [Online]. Available from http://www.astd.org/astd/Resources/eval roi community/return.htm

Phipps, R.A., \& Merisotis, J.P. (1989). What's the difference: A review of contemporary research on the effectiveness of distance learning in higher education. Washington, D.C.: The Institute for Higher Education Policy.

Phipps, R., Wilman, J., \& Mersater, J. (1998). Assessing quality in distance education. Washington, D.C.: Council on Higher Education.

Picciano, A.G. (2002). Beyond student perceptions: Issues of interaction, presence, and performance in an online course. Journal of Asynchronous Learning Networks, 6, $2-19$.

Powell, R., Conway, C., \& Ross, L. (1990). Effect of student predisposing characteristics on student success. Journal of Distance Education, 5, 5-19.

Presseisen, B.Z. (1992). A perspective on the evolution of cooperative thinking. In N. Davidson \& T. Worsham (Eds.), Enhancing thinking through collaborative learning. New York, Teachers College, Columbia University.

Rawlins, W. (1992). Friendship matters: Communication, dialectics, and the life course. New York: Aldine De Gruyter.

Ray, N., Tabor, S., \& Griggs, K. (2001). Web-based survey workshop. Western Decision Sciences Institute $30^{\text {th }}$ Annual Meeting. [Online]. Available from http://www.telecomm.boisestate.edu/research/Surveyresults_files/frame.html. 
Reeves, T. C., \& Reeves, P.M. (1997). Effective dimensions of interactive learning on the World Wide Web. In B.H. Kahn (Ed.), Web-based instruction. Englewood Cliffs, NJ: Educational Technology Publications.

Reimann, P., \& Bosnjak, M. (1998). Supporting hypertext-based argumentation skills. [Online]. Available from http://www.or.zumz.mannheim.de/bosnjak/publications/edmedia98/default.html.

Reio, T.G., Jr. (1997). Effects of curiosity on socialization-related learning and job performance in adults. Unpublished doctoral dissertation, Virginia Polytechnic Institute and State University, Blacksburg, Virginia.

Rezabeck, L.L., Meyers, D.C., Reiser, R.A., \& Edwin, E.O. (1992). Distance education: Perspectives from all sides of the desk. Paper presented at Annual Meeting of the Association for Educational Communications and Technology: Washington, D.C.

Rheingold, H. (1993). The virtual community. New York: Addison-Wesley.

Rice, R. (1984). Communication, research, and technology: The new media. Beverly Hills, CA: Sage.

Rice, R.E. (1993). Media appropriateness: Using social presence theory to compare traditional and new organizational media. Human Communication Research, 19, 451-484.

Richardson, J. (2001). Examining social presence in online courses in relation to students' perceived learning and satisfaction (Doctoral dissertation, University at Albany, State University of New York). Dissertations Abstract International. Ann Arbor, MI: UMI Press.

Richardson, J., \& Swan, K. (2003). An examination of social presence in online learning: Students' perceived learning and satisfaction. Paper presented the Annual Meeting of the American Educational Research Association. Seattle, WA.

Rifkind, L.J. (1992). Immediacy as a predictor of teacher effectiveness in the instructional television classroom. Journal of Interactive Television, 1, 31-38.

Ritchie, H., \& Newby, T.J. (1989). Classroom lecture/discussion vs. live televised instruction: A comparison of effects on student performance, attitude and interaction. The American Journal of Distance Education, 3, 36-45.

Roberson, T., \& Klotz, J. (2002). How can instructors and administrators fill the missing link in online instruction? Online Journal of Distance Learning Administration, 4. [Online]. Available from http://www.westga.edu/ distance/ojdla/winter54/roberson54.htm 
Roblyer, M.D., \& Ekhaml, L. (2000). How interactive are your distance courses? Online Journal of Distance Learning Administration, 3. [Online]. Available from http://www.westga.edu/ distance/roblyer32.html.

Rodriguez, D.E. (1995). Interaction in the ITESM's distance education system. Paper presented at the Invitational Research Conference in Distance Education: A research agenda.

Rogelberg, S.G., Luong, A., Sedeburg, M.E., \& Cristol, D.S. (2000). Employee attitude surveys: Examining the attitudes of noncompliant employees. Journal of Applied Psychology, 85, 284-293.

Rogers, E. (1995). Diffusion of Innovations (4 ${ }^{\text {th }}$ ed.). New York: Free Press.

Romiszowski, A.J., \& Ravitz, J. (1997). Computer-mediated communication. In C.R. Dills \& A.J. Romiszowski (Eds.), Instructional development paradigms, 745-768. Englewood Cliffs, NJ: Educational Technology Publications.

Rossing, B.E., \& Long, H.B. (1981). Contributions of curiosity and relevance to adult learning motivation. Adult Education, 32, 25-36.

Rossman, G., \& Rallis, S.F. (1998). Learning in the field: An introduction to qualitative research. Thousand Oaks, CA: Sage.

Rourke, L., Anderson, T., Garrison, R.D., \& Archer, W. (2001). Assessing social presence in an asynchronous text-based computer conferencing. Journal of Distance Education, 14, 51-70.

Rovai, A.P. (2002). Building a sense of community at a distance. International Review of Research in Open and Distance Learning, 3, 1-11.

Rovai, A.P. (2002). Development of an instrument to measure classroom community. The Internet and Higher Education, 5, 197-211.

Rubin, R.B., \& Palmgreen, E.A. (1994). Communication research measures: A sourcebook. New York: Guilford Press.

Russell, T. (1999). The no significant difference phenomenon. Chapel Hill, NC: Office of Instructional Telecommunications, North Carolina State University.

Russell, T. (2002). Significant difference. [Online]. Available from http://www.teleeducation.nb.ca/significantdifference/html. 
Ryan, M., Carlton, K.H., \& Ali, N.S. (1989). Evaluation of traditional classroom teaching methods versus course delivery via the World Wide Web. Nursing Education, 38, 272-277.

Saenz, B.L. (2002). Student perceptions of social presence and its value in an asynchronous Web-based Master's instructional program. Doctoral dissertation, Virginia Polytechnic Institute and State University, Blacksburg, VA. Dissertation Abstracts International. Ann Arbor, MI: UMI Press.

Saiedian, H. (1993). An interactive computer-based conferencing system to accommodate students' learning process. Journal of Educational Technology Systems, 21, 109-123.

Sanders, J., \& Wiseman, R. (1990). The effects of verbal and nonverbal teacher immediacy on perceived cognitive, affective, and behavioral learning. Communication Education, 39, 341-353.

Santos, L.M., \& DeOliveira, M. (1999). Internet as a freeway to foster critical thinking in lab activities. [Online]. Available from http://www.narst.org/conference/santosdeoliveira/santosdeoliveira.html.

Saye, J. (1997). Technology and educational empowerment: Students' perspectives. ETRD, 45, 23-26.

Scarce, R. (1997). Using electronic mail discussion groups to enhance students' critical; thinking skills. [Online]. Available from http://www.horizon.unc.edu/TS.html.

Schaefer, D.R., \& Dillman, D.A. (1998). Development of a standard email methodology: Results of an experiment. Public Opinion Quarterly, 62, 378-397.

Schieman, E., \& Jones, T. (1992). Learning at a distance: Issues for the instructional designer. Journal of Adult Education, 21, 3-13.

Schmidt, W.C. (1997). World-Wide Web survey research: Benefits, potential problems, and solutions. Behavior Research Methods, Instruments, \& Computers, 29, $274-$ 279 .

Schonlau, M., Fricker, R.D., \& Elliott, M.N. (2001). Conducting research surveys via email and the web. [Online]. Available from http://www.rand.org/publications/MR/MR1480/html.

Shale, D., \& Garrison, D.R. (1990). Introduction. In D. Shale (Ed.), Education at a distance, 1-6. Malabar, FL: Robert E. Kriger. 
Shannon, D.M., \& Bradshaw, C.C. (2002). A comparison of response rate, response time, and costs of mail and electronic surveys. The Journal of Experimental Education, 70, 179-192.

Sharon, S. (1980). Cooperative learning in small groups: Recent methods and effects on achievement, attitudes, and ethnic relations. Review of Educational Research, 50, 241-271.

Shih, L. (2004). Anatomy of asynchronous online learning: A study of factors that contribute to perceptions of social presence. Doctoral dissertation, State University of New York, Albany, NY. Dissertations Abstracts International. Ann Arbor, MI: UMI Press.

Sheehan, K.B., \& Hoy, M.G. (1999). Using email to survey Internet users in the United States: Methodology and assessment. Journal of Computer Mediated Communications, 4, 1-24.

Sheehan, K.B., \& McMillan, S.J. (1999). Response variation in email surveys: An exploration. Journal of Advertising Research, 39, 45-54.

Sherry, L. (1996). Issues in distance learning. Instructional Journal of Educational Telecommunications, 1, 337-365.

Short, J.A., Williams, E., \& Christie, B. (1976). The social psychology of telecommunications. London: John Wiley \& Sons, Ltd.

Sills, S.J., \& Song, C. (2001). Innovations in survey research: An application of Webbased surveys. Social Science Computer Review, 20, 22-30.

Simonson, M., Smaldino, S., Albright, M., \& Zvacek, S. (2000). Teaching and learning at a distance: Foundations of distance education. Upper Saddle River, NJ: Merrill.

Simsek, Z., \& Veiga, J. (2001). A primer on Internet organizational surveys. Organizational Research Methods, 4, 218-235.

Sisco, B.R. (1991). Setting the climate for effective teaching and learning. In R. Hiemstra (Ed.), Creating environments for effective adult learning, 41-50. San Francisco, CA: Jossey-Bass.

Slavin, R. (1983). Cooperative Learning. New York: Longman.

Slavin, R. (1990). Cooperative learning: Theory, research, and practice. Englewood Cliffs, NJ: Prentice Hall. 
Smith, M.C., \& Winking-Diaz, A. (2004). Increasing students' interactivity in an online course. The Journal of Interactive Online Learning, 2. [Online]. Available from http://www.ncolr.org.

Spears, R., \& Lea, M. (1992). Social influence and the influence of the "social" in computer-mediated communication. In M. Lea (Ed.), Contexts of computermediated communication, 30-65. New York: Harvester Wheatsheaf.

Sproull, L., \& Faraj, S. (1997). Atheism, sex and databases: The net as a social technology. In S. Kiesler (Ed.), Culture of the Internet, 35-52. Mahwah, NJ: Lawrence Erlbaum and Associates.

Sproull, L., \& Kiesler, S. (1991). Connections: New ways of working in the networked organization. Cambridge, MA: MIT Press.

Stanton, J.M. (1998). An empirical assessment of data collection using the Internet. Personnel Psychology, 51, 709-725.

Stoss, R.E. (2001). The new mailbox U.: Discarding standards in pursuit of a buck. U.S. News \& World Report, 37.

Swan, K. (2001). Immediacy, social presence, and asynchronous discussions. In J. Bourne \& Moore (Eds.), Elements of Quality Online Education, 3. Online and Babson Colleges: Sloan Center for Online Education, 2002.

Swan, K., Shea, P., Fredericksen, E., Pickett, A., Pelz, W., \& Maher, G. (2000). Building knowledge building communities: Consistencies, contact and communication in the virtual classroom. Journal of Educational Computing Research, 23, 359-383.

Swift, C.O., Wilson, J.W., \& Wayland, J. P. (1997). Interactive distance education in business: Is the new technology right for you? Journal of Education for Business, 73, 85-89.

Tabachnick, B.G., \& Fidell, L.F. (2000). Using multivariate statistics, $\left(4^{\text {th }}\right.$ ed.). Boston, MA: Allyn \& Bacon.

Tannen, D. (1990). You just don't understand me: Women and men in conversation. New York: William Morrow and Company.

Taylor, S., \& Lynn, P. (1998). The effect of a preliminary notification letter to a postal survey of young people. Journal of the Market Research Society, 2, 165-178.

Thompson, J.B. (1995). Social theory and the media. In D. Crowley \& D. Mitchell (Eds.), Communication Theory Today, 27-49. Oxford: Polity. 
Tinto, V. (1987). Leaving college: Rethinking the causes and cures of college attrition. Chicago, IL: University of Chicago Press.

Tinto, V. (1993). Leaving college: Rethinking the causes and cures of college attrition. $\left(2^{\text {nd }}\right.$ ed.). Chicago, IL: University of Chicago Press.

Tough, A. (1969). Some major reasons for learning. Self-concept in adult participation: Conference report and bibliography. (ERIC Document Reproduction Services No. ED 033252). 19-38.

Trentin, G., \& Benigno, V. (1997). Multimedia conferencing in education: Methodological and organizational considerations. Educational Technology, 37, 32-39.

Tu, C.H. (2000). From social learning theory to social presence in an online environment. Journal of Network and Computer Interactions, 23, 39-58.

Tu, C.H. (2002). The measurement of social presence in an online learning environment. International Journal on E-Learning, 1, 34-45.

Tu, C.H., \& Corry, M. (2001). Distance education: Research and practice. Washington, D.C.: Technology Advisory Committee at Graduate School of Education \& Human Development.

Tu, C.H., \& McIsaac, M. (2002). The relationship of social presence and interaction in online classes. The American Journal of Distance Education, 16, 131-150.

Tuckey, C.J. (1993). Computer conferencing and the electronic whiteboard in the United Kingdom: A comparative analysis. The American Journal of Distance Education, 7, 58-72.

Twigg, C.A. (1997). Is technology a silver bullet? Educom Review, (March/April). 2829.

Vrasidas, C., \& McIsaac, M.S. (1999). Factors influencing interaction in an online course. American Journal of Distance Education, 13, 22-36.

Vygotsky, L.S. (1978). Mind in society: The development of higher psychological processes. Cambridge, MA: Harvard University.

Vygotsky, L.S. (1986). Thought and language. Cambridge, MA: MIT Press.

Wagner, E.D. (1994). In support of a functional definition of interaction. American Journal of Distance Education, 8, 6-27. 
Wagner, E.D. (1997). Interactivity: From agents to outcomes. In T. Cyrs (Ed.), Teaching and learning at a distance: What it takes to effectively design, deliver, and evaluate programs, 71, 19-32. San Francisco, CA: Jossey-Bass.

Walker, K.B., \& Hackman, M.Z. (1991). Information transfer and nonverbal immediacy as primary predictors of learning and satisfaction in the televised course. Paper presented at the Annual Meeting of the Speech Communication Association, Atlanta, Georgia.

Walther, J. B. (1992). Interpersonal effects in computer-mediated interaction: A relational perspective. Communication Research, 21, 52-90.

Walther, J.B. (1994). Interpersonal effects in computer-mediated interaction. Communication Research, 21, 460-487.

Walther, J.B., \& Burgoon, J.K. (1992). Relational communication in computer-mediated interaction. Human Communication Research, 19, 50-88.

Ward, J. (1998). Community college student perceptions of online instruction experiences. Education at a Distance, 12, 6-14.

Warschauer, M. (1997). Computer-mediated collaborative learning theory and practice. The Modern Language Journal, 81, 1-16.

Wayland, J.P., Swift, C.O., \& Wilson, J.W. (1994). Student attitudes toward distance learning. In B. Engelland \& A.J. Bush (Eds.), Marketing Advances in Theory and Thought, 296-299. New Orleans, LA: Southern Marketing Association.

Webster, J., \& Hackey, P. (1997). Teaching effectiveness in technology-mediated distance learning. The Academy of Management Journal, 40, 1282-1309.

Wegerif, R. (1998). The social dimension of asynchronous learning networks. Journal of Asynchronous Learning Networks, 2. [Online]. Available from http://www.aln.org/publications/jaln/v2n1/v2n1_wegerif.asp.

Wellman, B. (1999). The network community: An introduction to networks in the global village. In B. Wellman (Ed.), Networks in the Global Village, 1-48. Boulder, CO: Westview Press.

Wenger, E. (1998). Communities of practice: Learning, meaning and identity. New York: Cambridge University.

Westbrook, T.S. (1999). Changes in student attitudes toward graduate instruction via Web-based delivery. The Journal of Continuing Higher Education, 47, 32-38. 
White, K. (2000). Face to face in the online classroom. In K. White and B. Weight (Eds.), The online teaching guide: A handbook of attitudes, strategies, and techniques for the virtual classroom, 1-12. Needham Heights, MA: Allyn \& Bacon.

Whitworth, J.M. (1998). Looking at distance learning through both ends of the camera. Paper presented at the Annual Meeting of the National Association for Research on Science Teaching, San Diego, CA.

Wiener, M. \& Mehrabian, A. (1968). Language within language: Immediacy, a channel in verbal communication. New York: Appleton-Century-Crofts.

Williams, M. Paprock, K., \& Covington, B. (1999). Distance learning: The essential guide. Thousand Oaks, CA: Sage.

Winiecki, D.J. Keeping the thread: Adapting conversational practice to help distance students and instructors manage discussions in an asynchronous learning network. DEOSNEWS, 9. [Online]. Available from http://www.ed.psu.edu/ASCDE.html.

Winn, W. (1993). Perception principles. In M. Flemming \& W.H. Levie (Eds.), Instructional message design, ( $2^{\text {nd }}$ ed.), 55-126. Englewood Cliffs, NJ: Educational Technology Publications.

Wolcott, L. (1996). Distant, but not distanced: A learner-centered approach to distance education. TechTrends, 41, 23-27.

Woods, R.H., \& Baker, J.D. (2004). Interaction and immediacy in online learning. International Review of Research in Open and Distance Learning, 8, 1-11.

Woods, R., \& Ebersole, S. (2003). Becoming a communal architect in the online classroom: Integrating cognitive and affective learning for maximum effect in web-based learning. Online Journal of Distance Learning Administration, 61. [Online]. Available from http://www.westga.edu/ distance/ojdla/spring61/woods61.htm.

Wulf, S., Hanor, J., \& Bulik, R.J. (2000). The roles and interrelationships of presence, reflection, and self-directed learning in effective World Wide Web-based pedagogy. In R.A. Cole (Ed.), Issues in Web-based pedagogy: A critical primer, 143-160.

Wynia, L. (2000). A comparison of traditional and interactive television classroom environments and their impact on student perceived success and satisfaction. Doctoral dissertation, University of South Dakota, 2000. Dissertation Abstracts International, $A B(3), 956$. 
Yun, G.W., \& Trumbo, C.W. (2000). Comparative response to a survey executed by post, email, and Web form. Journal of Computer-Mediated Communication, 6. [Online]. Available from http://www.ascusc.org/jemc/html.

Zielinski, D. (2000). Can you keep learners online? Training, 37, 64-75.

Zirkin, B.G., \& Sumler, D.E. (1995). Interactive or non-interactive? That is the question!!! An annotated bibliography. Journal of Distance Education, 10, 95112. 


\title{
Appendix A
}

\author{
Advertisement \\ Pre-notification Email to all Students Enrolled in Online Courses \\ (Example)
}

From: $\quad$ Susan J. Crim via Delphi Center of Teaching and Learning

To: $\quad$ Emails of students enrolled online

Date: $\quad$ Fall 2005

Subject: $\quad$ Upcoming Research Study on Social Presence

Permission has been granted by your course instructor to notify you of an upcoming research study that will involve an invitation to participate in an online survey being conducted by the College of Education and Human Development at the University of Louisville by Dr. Thomas G. Reio, Jr. and Susan J. Crim, a doctoral candidate at the University of Louisville. This is a pre-notification email to inform you that you will be receiving the details of how to access the Web-based survey within a few days. The survey consists of four sections and it may take approximately 15-20 minutes to complete. You must be 18 years or older to participate in this research study.

The survey is designed to seek your feedback on the perception of social presence and satisfaction with learning in an online course. Your feedback is valuable and may be used to enhance the understanding of how student needs, experiences, and perspectives may influence an optimal learning environment for online distance students. This insight could assist educators and instructional designers in planning, designing, organizing, managing, and delivering quality Web-based instruction.

Data collection for this study will be obtained through a Web-based survey launched by the Delphi Center through the course on Blackboard and GroupWise email. Although absolute confidentiality cannot be guaranteed, confidentiality will be protected to the extent permitted by law. The server hosting the survey and the raw data is one that is secured within the University of Louisville. The Institution Review Board (IRB) has reviewed this study. Should the data be published, you will not be identified by name. Once this research study is completed, the results will be analyzed and reported in aggregate form.

Your participation in this study is voluntary and anonymity is ensured to the extent permitted by law. You may refuse to answer any questions that you are uncomfortable 
with and discontinue participation at any time without losing any benefits to which you are otherwise entitled.

Should you have any questions, you may call the investigator at (502) 852-1229 or contact by email at sjbail02@, louisville.edu.

By returning the web-based survey, you are indicating your willingness to participate freely in this research study.

Thank you very much for considering the invitation to participate in this study. 


\section{Appendix B}

\section{Notification of Survey to all Students Enrolled in Online Courses (Example)}

From: $\quad$ Susan J. Crim via Delphi Center for Teaching and Learning

To: $\quad$ Emails of students enrolled online

Date: $\quad$ Fall 2005

Subject: $\quad$ Research Study on Social Presence

You are invited to participate in a research study being conducted by the College of Education and Human Development at the University of Louisville by Dr. Thomas G. Reio, Jr. and Susan J. Crim, a doctoral candidate at the University of Louisville. You must be 18 years or older in order to participate in this research study.

The web-based survey is designed to measure student perceptions of social presence and satisfaction with learning in an online course. Your responses may assist in an effort to improve the development of online courses that are effective in the promotion of learning. The survey is divided into four (4) sections and it will take approximately 20 minutes to complete. We would appreciate your reply within 2 weeks.

To begin, clink on this link

http://www.zoomerang.com/survey.zgi?p=WEB224NR22G4GH to gain entry to the survey.

If you are unable to follow this link, copy and paste this web address using your web browser.

When you have completed the survey, click on the SUBMIT button at the end of the survey.

This is an anonymous survey and your responses will be kept confidential to the extent permitted by law although absolute confidentiality cannot be guaranteed. The server hosting the survey and the raw data is one that is secured with the University of Louisville. The sponsor and the Human Subjects Protection Program Office (HSPO), and 
the Institutional Review Board (IRB) may inspect the research records for this study. Should the data be published, you will not be identified by name. Once this research study is completed, the results will be analyzed and reported in aggregate form.

Your participation is voluntary and you may refuse to answer any questions that you are uncomfortable with or choose to stop participating at any time without losing any benefits to which you are otherwise entitles. As with any research there is always the possibility of unforeseen risks. There are no foreseeable risks associated with this study.

Should you have any questions, you may call the investigator at (502) 852-1229 or contact by email sjbail02@louisville.edu. If you have any questions or concerns about your rights as a research subject, concerns or complaints about the research staff, you may call the HSPO (502) 852-5188, and they will put you in touch with the appropriate chair of the Institutional Review Board Committee to discuss the matter.

Responding to and submitting this survey electronically will indicate your willingness to participate in this research study.

Thank you for your consideration and time given to our invitation to participate in this study.

Sincerely,

Thomas G. Reio, Jr.

Susan J. Crim 


\section{Appendix C}

\section{Social Presence Survey for Online Courses}

The purpose of this survey is to determine learners' perceptions of social presence in their online courses. The researcher would like to know what students think about their online courses and what aspects they perceive as facilitating their learning in those courses, such as the presence of others. This survey is provided in hopes of gathering more specific information regarding your perceptions so that course instructors may be able to improve their online courses.

Your participation in this process is strictly voluntary and participants are ensured anonymity and confidentiality to the extent permitted by law. There are four sections to this survey. Responding to and submitting this survey electronically will indicate your willingness to participate. It takes about 15-20 minutes to complete all sections. Please answer each item and complete this online survey within 2 weeks. Thank you for your assistance.

The following descriptions apply to the survey:

Online community- Class participation, discussion, and a high level of instructor-learner and learner-learner interaction is encouraged.

Social presence- A sense of belonging in a course or group and the ability to interact with others although physical contact is not available.

\section{Section I.}

Course Name

Course Instructor

Your Age__ Your Gender

Approximate number of college credits completed

Online Experience:

If this is not your first online course, how many online courses have you completed?

What are your reasons for taking the course in the online format? Please check all that apply.

course only offered online

like the convenience of online

required for major

enjoy interaction with others online

curiosity about the subject

other (Briefly explain) 


\section{Section II.}

Please read each statement carefully; then indicate the degree to which you Agree or Disagree with the statement as it relates to your online experience overall for this course. (1= strongly disagree. $2=$ disagree. $3=$ somewhat disagree. $4=$ somewhat agree. $5=$ agree. $6=$ strongly agree)

\section{Questions}

1. Online or web-based communication is an excellent medium for social interaction.

123456

2. I felt comfortable conversing through this online medium.

123456

3. I felt comfortable introducing myself in this course.

123456

4. The introductions enabled me to form a sense of online community.

123456

5. The instructor created a feeling of an online community.

123456

6. I felt comfortable participating in course discussions.

123456

7. The instructor facilitated discussions in this course.

123456

8. I felt comfortable interacting with other participants in the course.

123456

9. I felt that the online messages were impersonal (no human characteristics).

123456

10. I felt that my point of view was acknowledged by others in this course.

11. I felt this medium was an informal and casual way to communicate.

12. I felt the online messages were able to convey feeling and emotion.

13. The replies to my messages were immediate.

14. Users within this course are normally responsive to messages.

15. The language participants use in online communications is stimulating.

16. It is difficult to express what I want to communicate online.

17. The language used to express oneself online is meaningful.

18. The language used to express oneself online is easily understood.

19. I am comfortable participating, if I am familiar with the topics.

20. I am uncomfortable participating, if I am not familiar with the topics.

21. I am comfortable communicating with a person who is familiar to me.

22. I am uncomfortable communicating with a person who is unfamiliar to me.

23. I was able to form distinct impressions of some course participants.

24. My level of learning that took place in this course was of the highest quality.

25. I was pleased with the individualized feedback on my course assignments.

26. The individualized feedback received was constructive.

27. Overall the instructor for this course met my expectations.
123456

123456

123456

123456

123456

123456

123456

123456

123456

123456

123456

123456

123456

123456

123456

123456

123456

123456 
28. Overall, this course met my learning expectations.

29. I am satisfied with the support I received in this course.

30. I am satisfied with my learning experience in this course.

123456

31. Generally, I have had a positive learning experience in this course.

123456

32. I am satisfied with the feedback I have received about my performance in this online course.

123456

33. It is important to receive personalized communication from the instructor.

123456

34. It is important to receive individualized feedback from the instructor.

123456

35. It is important to feel a sense of concern from the instructor.

123456

36. It is important to have frequent interaction with the instructor.

123456

37. It is important to establish friendships with other students.

123456

38. It is important to receive individualized feedback from other students.

123456

39. It is important to receive personalized communication from other students.

123456

40. It is important to feel that you are part of an online learning community.

123456

41. I often think about quitting this online course.

123456

42. It is likely that you will actively look for a new online course to take next semester.

123456

43. I will probably look for a new online course to take within the next year.

123456

44. It is not likely that I will take another online course.

123456

\section{Section III.}

The following indicator statements examine the specific activities within your course. For each of the following statements, indicate the degree to which you Agree or Disagree with the statement as it reflects your experience for this course that corresponds with each course activity noted across the top of the table. (Note: If your course does not contain a particular activity then you should respond with "NA" for not applicable. 
( $1=$ strongly disagree. $2=$ disagree. $3=$ somewhat disagree. $4=$ somewhat agree. $5=$ agree. $6=$ strongly agree. $7=$ NA. $)$

\begin{tabular}{|c|c|c|c|c|c|}
\hline & $\begin{array}{l}\text { Course } \\
\text { Activities }\end{array}$ & & & & \\
\hline Indicator Statements & $\begin{array}{l}\text { Class } \\
\text { Discussions }\end{array}$ & $\begin{array}{l}\text { Individual } \\
\text { Projects }\end{array}$ & $\begin{array}{l}\text { Group } \\
\text { Projects }\end{array}$ & $\begin{array}{l}\text { Reading } \\
\text { Assignments }\end{array}$ & $\begin{array}{l}\text { Writing } \\
\text { Assignments }\end{array}$ \\
\hline $\begin{array}{l}\text { 1. Quality of learning for this activity } \\
\text { was excellent. }\end{array}$ & & & & & \\
\hline $\begin{array}{l}\text { 2. I gained more interest in the subject } \\
\text { through this activity. }\end{array}$ & & & & & \\
\hline $\begin{array}{l}\text { 3. My skill in critical thinking } \\
\text { increased through this activity. }\end{array}$ & & & & & \\
\hline $\begin{array}{l}\text { 4. I became more confident in } \\
\text { expressing ideas through this activity. }\end{array}$ & & & & & \\
\hline $\begin{array}{l}\text { 5. I felt comfortable } \\
\text { conversing through } \\
\text { this activity. }\end{array}$ & & & & & \\
\hline $\begin{array}{l}\text { 6. Online education } \\
\text { is an excellent } \\
\text { medium for social } \\
\text { interaction as } \\
\text { demonstrated by } \\
\text { this activity. }\end{array}$ & & & & & \\
\hline $\begin{array}{l}\text { 7. This activity } \\
\text { enabled me to } \\
\text { form a sense of } \\
\text { online community. }\end{array}$ & & & & & \\
\hline $\begin{array}{l}\text { 8. I felt comfortable } \\
\text { participating with } \\
\text { others in this } \\
\text { activity. }\end{array}$ & & & & & \\
\hline $\begin{array}{l}\text { 9.My point of view was acknowledged } \\
\text { by other } \\
\text { participants during this activity. }\end{array}$ & & & & & \\
\hline $\begin{array}{l}\text { 10. I was able to form distinct } \\
\text { individual impressions of some course } \\
\text { participants during this activity. }\end{array}$ & & & & & \\
\hline
\end{tabular}

\section{Section IV.}

1. Which of the five (5) activities above did you find most beneficial to your learning and why?

2. To what degree did the course activities and assignments facilitate online communication and interaction? Please explain. 
3. How much interaction did you have with your instructor (e.g. significant, moderate, sufficient, or lacking)? Please describe.

4. Would you say the type and amount of learner-learner interaction was adequate for this course? Please describe.

5. As a result of your experiences within this course, would you consider taking another web-based course? Briefly explain.

6. Based upon your experience within this online course, are there any comments you would like to add? 


\section{Appendix D}

Demographic Information

Other reasons for taking online courses

Frequency

$\%$ of Total

Geographic distance

17

12

Job/ Military responsibilities

10

Could not enroll in campus course

Family

Program only offered online

Time and convenience

Cost of travel

Young children; just had a baby

Medical reasons; physical impairment

Curiosity about online classes

7

6

6

3

3

3

1

$25.0 \%$

$17.6 \%$

$14.7 \%$

$10.3 \%$

$.8 \%$

$.8 \%$

$.4 \%$

$.4 \%$

$.4 \%$

$.1 \%$

Totals

68 


\section{CURRICULUM VITAE}

NAME: $\quad$ Susan Jill Crim

ADDRESS: 12925 Crestmoor Circle

Prospect, Kentucky 40059

EDUCATION: $\quad$ B.S., Health Education and Dental Hygiene

East Tennessee State University

1970-1974

M.S., Adult Education

University of Tennessee, Knoxville

1983-1985

Ph.D., Education, Leadership, and Human Resource Development University of Louisville

2002-2006

EMPLOYMENT: Assistant Professor and Director of Dental Hygiene

University of Louisville School of Dentistry

2001-Present

American Dental Association Commission on Dental Accreditation Consultant and Staff Representative

1984-Present

AWARDS: Phi Kappa Phi Honor Society

2006

Omicron Kappa Upsilon Honor Society in Dentistry

2004

Kappa Delta Pi Honor Society in Education

2000

Who's Who in Medicine and Health Care

1999 
American Dental Hygienists' Association Award for Excellence 1998

Who's Who of the Year by American Institute Board of Research 1994

Sigma Phi Alpha Dental Hygiene Honor Society 1990

Pi Lambda Theta Honor Society in Education 1984

Alpha Lambda Delta Honor Society in Education 1972

PROFESSIONAL SOCIETIES: $\quad$ American Dental Education Association Council on Allied Dental Program Directors, Chair 2003-2006

Dental Hygiene Curriculum Guidelines Committee $1992 / 2004$

American Dental Hygienists' Association 1974-Present

Council on Dental Education, Chair 1994-1998
PUBLICATIONS: Commission on Dental Accreditation Workbook (contributing author)
A Guide for Developing an Accredited Dental Hygiene Program 1998

NATIONAL MEETING PRESENTATIONS: Association of Human Resource Development Conference Columbus, $\mathrm{OH}$ Article Presentation- The emergence of social presence as an overlooked factor in asynchronous online learning. Reio, T.G., Jr. \& Crim, S. 2006

Oral Health 2000 Fifth National Consortium Panel Speaker Atlanta, GA

1997 
INVITED PRESENTATIONS:

American Dental Hygienists' Assoc.

Professional Issues Forum 1997 